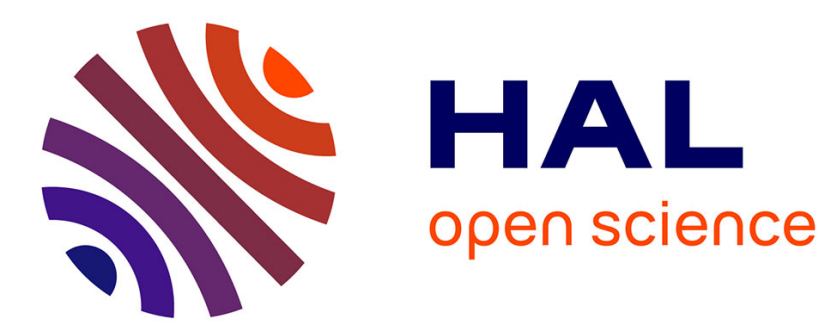

\title{
Limit states of internal wave beams
}

Bruno Voisin

\section{To cite this version:}

Bruno Voisin. Limit states of internal wave beams. Journal of Fluid Mechanics, 2003, 496, pp.243-293. 10.1017/S0022112003006414 . hal-00259838

\section{HAL Id: hal-00259838 \\ https://hal.science/hal-00259838}

Submitted on 30 Mar 2011

HAL is a multi-disciplinary open access archive for the deposit and dissemination of scientific research documents, whether they are published or not. The documents may come from teaching and research institutions in France or abroad, or from public or private research centers.
L'archive ouverte pluridisciplinaire HAL, est destinée au dépôt et à la diffusion de documents scientifiques de niveau recherche, publiés ou non, émanant des établissements d'enseignement et de recherche français ou étrangers, des laboratoires publics ou privés. 


\title{
Limit states of internal wave beams
}

\author{
By B. VOISIN \\ Laboratoire des Écoulements Géophysiques et Industriels, CNRS-UJF-INPG, BP 53, \\ 38041 Grenoble Cedex 9, France \\ Bruno.Voisin@hmg.inpg.fr
}

(Received 9 September 1998 and in revised form 17 July 2003)

Internal gravity waves propagate away from a localized monochromatic disturbance inside beams, which develop around a St Andrew's Cross in two dimensions and around a double cone in three dimensions. The structure of the beams depends on three mechanisms, which couple together the different directions of propagation of the waves within the fluid. These mechanisms are associated with the size of the disturbance, the start-up of the motion and the viscosity of the fluid, respectively. The present paper considers each mechanism in isolation, for three-dimensional generation. The analysis is asymptotic and relies on far-field and large-time approximations. For each mechanism, three expressions of the waves are found: one, exact for an extended disturbance, that involves all the wavenumber vectors satisfying the dispersion relation; and two others, respectively uniform and non-uniform asymptotic expansions, that involve only the wavenumber vectors associated with group velocity vectors pointing toward the observer. For each mechanism, profiles of pressure and velocity are presented. A new time-independent characterization of the waves is introduced, in terms of the intensity or average energy flux; it is applied to the definition of the beam width. For an extended disturbance this width is a constant, the diameter of the disturbance. For an impulsive start-up the width increases linearly with the distance from the disturbance, and decreases in inverse proportion to the time elapsed since the start-up. For a viscous fluid the width increases as the one-third power of the distance. In all three cases, for a disturbance of multipolar order $2^{n}$, at constant strength, the power output varies in inverse proportion to the $(2 n+1)$ th power of the beam width.

\section{Introduction}

A major difficulty of internal wave theory is, for linear waves in a uniformly stratified Boussinesq fluid otherwise at rest, the absence of any length scale. Shorter waves may accumulate at particular places and times, leading to a divergence of the amplitude and to arbitrarily fast variations of the phase. In the present paper we investigate how, in such circumstances, meaning is restored to the theory by taking into account various additional phenomena usually neglected to a first approximation. The simplest possible wave field is considered, that generated by localized monochromatic excitation.

The literature abounds in studies of this field. Experiments involve production by oscillating bodies, and visualization and measurement by shadowgraph (Görtler 1943), schlieren method (Mowbray \& Rarity 1967), particle tracking (Thomas \& Stevenson 1972; Gordon \& Stevenson 1972), interferometry (Laws, Peat \& Stevenson 
1982; Peters 1985), Moiré method (Ivanov 1989), combination of interferometry and conductimetry (Makarov, Neklyudov \& Chashechkin 1990; Kistovich, Neklyudov \& Chashechkin 1990), and coloured layering (Gavrilov \& Ermanyuk 1997), for horizontal circular cylinders; or particle tracking (McLaren et al. 1973), for spheres. More recently the synthetic schlieren method, where optical imaging is replaced by digital processing, has been applied to circular (Sutherland et al. 1999, 2000; Dalziel, Hughes \& Sutherland 2000) and elliptical (Sutherland \& Linden 2002) horizontal cylinders, and to spheres (Onu, Flynn \& Sutherland 2003; Flynn, Onu \& Sutherland 2003; Sutherland, Flynn \& Onu 2003). Alternative production mechanisms have also been used, either inviscid such as an articulated paddle, investigated numerically by Javam, Imberger \& Armfield (2000), or viscous such as an inclined plate oscillating along its plane, a vertical circular cylinder oscillating along its axis and a horizontal disk oscillating around a vertical axis, investigated experimentally by Il'inykh, Kistovich \& Chashechkin (1999), Il'inykh, Smirnov \& Chashechkin (1999), Chashechkin, Kistovich \& Il'inykh (2000) and Chashechkin, Kistovich \& Smirnov (2001).

Theoretical studies are numerous too. As a rule, the stratification being characterized by the buoyancy frequency $N$, the dispersion relation implies that energy radiated at a given frequency $\omega_{0}$ can only propagate if $\omega_{0}<N$, at the angle $\theta_{0}=\arccos \left(\omega_{0} / N\right)$ to the vertical. Accordingly, the waves from a localized monochromatic disturbance are found in two dimensions on a St Andrew's Cross with branches intersecting at the disturbance, and in three dimensions on a double cone with apex at it (Lighthill $1978, \S 4.4)$; hence the names, sometimes used, of cross waves and conical waves.

No information on the phase or amplitude of the waves is obtained in this way, and calculating explicitly the response of the fluid to point monochromatic excitation does not help much from that point of view, as seen e.g. by Voisin (1991). The explanation for this failure lies in an argument originally put forward by Peters (1985): since, at a given frequency, energy propagates at a specific angle to the vertical, conversely each direction of a stratified fluid, inclined at the angle $\theta_{0}$ to the vertical say, is an independent oscillator resonating at its natural frequency $\omega_{0}=N \cos \theta_{0}$. In the absence of any mechanism coupling the oscillators together, energy injected at the frequency $\omega_{0}$ at a certain point will only propagate along the resonating oscillator, of inclination $\theta_{0}=\arccos \left(\omega_{0} / N\right)$, stretching out through that point; the phase variations imposed at the excitation will not be transmitted to any other direction, neither will be the energy input, and no wave field will be created.

Three such mechanisms are available: forcing along the surface of a source of non-zero size; existence within the fluid, prior to the establishment of a steady state, of transients excited by the start-up of the motion; and viscous momentum transfer. All three mechanisms have been considered already in the theoretical literature, either separately or in combination.

The fundamental reference on this topic is $\S 4.10$ of Lighthill (1978), where steady monochromatic waves are derived for arbitrary two- and three-dimensional source terms in the wave equation, in either inviscid or viscous fluids. In addition Gorodtsov \& Teodorovich (1986) have calculated the inviscid power output of the same sources. Unfortunately these results are difficult to apply quantitatively, for lack of a known representation of any realistic forcing - generally an oscillating body as a source term in the wave equation, and also because Lighthill's derivation involves asymptotic approximations - an explicit far-field assumption $r \rightarrow \infty$, with $r$ the distance from the source, in three dimensions, and an implicit low-viscosity assumption - with unspecified domains of validity. Accordingly, most investigations 
rely on another angle of attack, the direct solution of the wave equation for the particular forcing at stake.

The first coupling mechanism - the size of the source - has been considered by solving the boundary-value problem associated with the oscillation of various bodies: a flat (Hurley 1969), circular (Hurley 1972; Appleby \& Crighton 1986) or elliptical (Hurley 1997; Hurley \& Hood 2001) horizontal cylinder, a sphere (Hendershott 1969; Appleby \& Crighton 1987; Voisin 1991) and a flat (Krishna \& Sarma 1969) or generic (Sarma \& Krishna 1972; Lai \& Lee 1981) spheroid. Waves are obtained, confined to a shell delimited by the St Andrew's Crosses, or characteristic double cones, tangent to the oscillating body above and below. Unfortunately, the solution is not of the form, an integral in wavenumber space, suitable for extension to unsteady or viscous situations.

The second coupling mechanism - the start-up of the motion - has been studied for a three-dimensional point source switched on impulsively: Makhortykh \& Rybak (1990) proved that the waves are confined to a shell around the characteristic double cone with apex at the source, and Simakov (1993) calculated the temporal rate of increase of the waves on this cone. Unfortunately, in neither case was the structure of the waves investigated inside the shell.

The third coupling mechanism - the viscosity of the fluid - has been considered for a two-dimensional point source in a viscous fluid by Thomas \& Stevenson (1972), Gordon \& Stevenson (1972), Ramachandra Rao \& Balan (1977), Makarov et al. (1990) and Kistovich \& Chashechkin (1994). Again, waves are obtained, confined to a shell around the St Andrew's Cross with branches intersecting at the source. Unfortunately, the analysis involves an approximation, of boundary-layer type in real space (Thomas \& Stevenson 1972; Gordon \& Stevenson 1972) and small-wavenumber type in Fourier space (Makarov et al. 1990; Kistovich \& Chashechkin 1994), with unspecified domain of validity. Ramachandra Rao \& Balan (1977), by the numerical evaluation of the Fourier integral representing the waves, showed that the approximation amounts to a far-field assumption.

The combination of the three coupling mechanisms has been considered in pairs. The combination of the size of the source with the start-up of the motion has been studied, in three dimensions, by Hendershott (1969) for a pulsating sphere and Simakov (1994) for an arbitrary distribution of singularities on a curve or surface. In both cases, only the temporal rate of increase of the waves on the characteristic double cones tangent to the source above and below was calculated, not the waves themselves between the two cones.

More attention has been devoted to the combination of the size of the source with the viscosity of the fluid, in two dimensions, for either discrete (Stevenson, Bearon \& Thomas 1974) or continuous (Chang \& Stevenson 1975) horizontal distributions of singularities, and for a horizontal cylinder oscillating in a fluid considered either homogeneous (Ivanov 1989; Makarov et al. 1990) or stratified (Hurley \& Keady 1997; Hurley \& Hood 2001) at the cylinder's surface. A free-slip boundary condition is prescribed, which amounts to assuming that only the propagation of the waves is affected by viscosity while their generation remains inviscid; the consistency of this assumption stems from the fact that the boundary-layer, or small-wavenumber, approximation involved in the calculation of the waves is also a low-viscosity approximation.

Chashechkin and coworkers have recently adopted the opposite point of view, by looking at situations in which viscosity though small is alone responsible for wave generation, through the formation of oscillatory boundary layers. These situations 
include an inclined plate oscillating along its plane (Chashechkin \& Kistovich 1997; Kistovich \& Chashechkin 1999a,b; Chashechkin et al. 2001), a vertical plate oscillating vertically (Kistovich \& Chashechkin 2002), a vertical cylinder oscillating along its axis, around it or horizontally (Chashechkin et al. 2001; Kistovich \& Chashechkin 2001a), and a horizontal disk oscillating around a vertical axis (Kistovich \& Chashechkin 1999 c). A no-slip condition is prescribed, freeing the analysis from the approximation associated with the free-slip condition; however, another approximation is introduced, which consists in prescribing the no-slip condition not only at the actual surface of the oscillating body, but at a fictitious surface encompassing it and extending up to infinity: a whole plane for the plate, actually of finite width, and for the disk, actually of finite radius, and a whole cylindrical shell for the cylinder, actually of finite height. An iterative procedure has been introduced for removing this approximation (Kistovich \& Chashechkin 2001b).

It appears eventually that the different aspects of monochromatic internal wave generation, though all considered previously in the literature, have not been covered consistently in a way allowing, for example, the quantitative application of the theory to a given laboratory experiment. The present paper aims at initiating this coverage, by studying the effect of each coupling mechanism in isolation, for threedimensional generation by an arbitrary source term in the wave equation. Whenever possible, for each mechanism the analysis is developed first in real space and time, then in Fourier space and time, so as to build a common mathematical framework allowing the study, in forthcoming papers, of all three mechanisms together, for either two- or three-dimensional generation. A companion series of papers is planned, considering the determination of the source terms equivalent to the most common sources of internal waves in the laboratory: oscillating spheres and circular cylinders.

The present paper starts in $\S 2$ with a derivation of the wave equation that takes all three mechanisms into account. It continues in $\S 3$ with a calculation of the waves without any such mechanism, namely for a point monopole oscillating steadily in an inviscid fluid. Sections 4-6 study the modifications to the calculation when each mechanism is taken into account separately, respectively the size of the source, the start-up of the motion and the viscosity of the fluid. Section 7 pays more attention to one quantity of experimental interest, the width of the wave beams, and $\S 8$ summarizes the main results of the paper together with their applications.

\section{Wave equation}

We consider small disturbances in a viscous incompressible uniformly stratified Boussinesq fluid otherwise at rest. Position is denoted by $\boldsymbol{x}=(x, y, z)$, time by $t$ and velocity by $\boldsymbol{u}=(u, v, w)$. The $z$-axis is directed vertically upwards along the unit vector $\boldsymbol{e}_{z}$, and a subscript h will indicate a horizontal projection so that, for example, $\boldsymbol{x}_{\mathrm{h}}=(x, y, 0)$. In the undisturbed fluid the density $\rho_{0}(z)$ decreases exponentially with height yielding a constant buoyancy frequency $N=\left[-\left(g / \rho_{0}\right)\left(\mathrm{d} \rho_{0} / \mathrm{d} z\right)\right]^{1 / 2}$, and the pressure $p_{0}(z)$ also decreases exponentially according to the hydrostatic law $\mathrm{d} p_{0} / \mathrm{d} z=-\rho_{0}(z) g$, with $\boldsymbol{g}=-g \boldsymbol{e}_{z}$ the acceleration due to gravity. In the presence of a source of mass releasing the volume $q$ of fluid per unit volume per unit time, the departures $\rho, p$ and $\boldsymbol{u}$ from the undisturbed values $\rho_{0}, p_{0}$ and $\mathbf{0}$ are governed by the 
linearized equations of motion

$$
\begin{gathered}
\rho_{0} \frac{\partial \boldsymbol{u}}{\partial t}=-\nabla p+\rho \boldsymbol{g}+\rho_{0} \nu \nabla^{2} \boldsymbol{u}, \\
\nabla \cdot \boldsymbol{u}=q, \\
\frac{\partial \rho}{\partial t}=\rho_{0} \frac{N^{2}}{g} w,
\end{gathered}
$$

respectively, the Navier-Stokes equation, the equation of continuity and the equation of state. In these equations, consistent with the Boussinesq approximation, the undisturbed density $\rho_{0}$ and the kinematic viscosity $v$ are constant.

\subsection{Wave/vortex decomposition}

Viscosity diffuses vorticity, with diffusivity $v$, invalidating the derivation of the wave equation by Voisin (1991), based on the conservation of vertical vorticity. A new derivation is required, that separates the dynamics of the vortical, non-propagating, part of the motion from that of the wavy, propagating, part. We proceed by adapting the wave/vortex decomposition of strongly stratified flow, discussed e.g. by Staquet \& Riley (1989) and Riley \& Lelong (2000).

First, the application of the Helmholtz theorem to the horizontal velocity gives

$$
\boldsymbol{u}=\nabla_{\mathrm{h}} \phi+\nabla_{\mathrm{h}} \times\left(\psi \boldsymbol{e}_{z}\right)+w \boldsymbol{e}_{z} .
$$

The horizontal component of (2.1) is reduced to the combination of a single equation for the horizontal streamfunction $\psi$, namely

$$
\left(\frac{\partial}{\partial t}-v \nabla^{2}\right) \psi=0
$$

and a relation between $p$ and the horizontal velocity potential $\phi$, namely

$$
p=-\rho_{0}\left(\frac{\partial}{\partial t}-v \nabla^{2}\right) \phi .
$$

The subsequent elimination of $\rho$ between (2.3) and the vertical component of (2.1) yields an equation for $\phi$ and $w$, namely

$$
\left(\frac{\partial}{\partial t}-v \nabla^{2}\right) \frac{\partial}{\partial t} \frac{\partial}{\partial z} \phi=\left[\left(\frac{\partial}{\partial t}-v \nabla^{2}\right) \frac{\partial}{\partial t}+N^{2}\right] w
$$

of solution

$$
\phi=\left[\left(\frac{\partial}{\partial t}-v \nabla^{2}\right) \frac{\partial}{\partial t}+N^{2}\right] \chi, \quad w=\left(\frac{\partial}{\partial t}-v \nabla^{2}\right) \frac{\partial}{\partial t} \frac{\partial}{\partial z} \chi,
$$

with $\chi$ an arbitrary scalar function; the completeness of this solution follows from gauge invariance. The remaining (2.2) becomes

$$
\left[\left(\frac{\partial}{\partial t}-v \nabla^{2}\right) \frac{\partial}{\partial t} \nabla^{2}+N^{2} \nabla_{\mathrm{h}}^{2}\right] \chi=q
$$

a single equation allowing the determination of $\chi$ given any forcing $q$.

In this way, the velocity field has been separated into two independent parts: one, containing all the vertical vorticity, described by the function $\psi$ satisfying (2.5) and to which the velocity, pressure and density are related by

$$
\boldsymbol{u}_{\mathrm{v}}=-\boldsymbol{e}_{z} \times \nabla \psi, \quad p_{\mathrm{v}}=0, \quad \rho_{\mathrm{v}}=0 ;
$$


and the other, containing strictly horizontal vorticity, described by the function $\chi$ satisfying (2.9) and to which the velocity, pressure and density are related by

$$
\begin{gathered}
\boldsymbol{u}_{\mathrm{w}}=\left[\left(\frac{\partial}{\partial t}-\nu \nabla^{2}\right) \frac{\partial}{\partial t} \nabla+N^{2} \nabla_{\mathrm{h}}\right] \chi, \\
p_{\mathrm{w}}=-\rho_{0}\left[\left(\frac{\partial}{\partial t}-\nu \nabla^{2}\right) \frac{\partial}{\partial t}+N^{2}\right]\left(\frac{\partial}{\partial t}-\nu \nabla^{2}\right) \chi, \\
\rho_{\mathrm{w}}=\rho_{0} \frac{N^{2}}{g}\left(\frac{\partial}{\partial t}-\nu \nabla^{2}\right) \frac{\partial}{\partial z} \chi .
\end{gathered}
$$

Equation (2.5) is the usual viscous diffusion equation, and (2.9) the usual internal wave equation modified by the addition of viscosity. Accordingly, the mathematical decomposition (2.10)-(2.11) is indeed a physical decomposition of the motion, into a vortical component of streamfunction $\psi$ and a wavy component of wave function $\chi$. With notation ' $\psi$ ' this latter function has been introduced in inviscid fluids by Gorodtsov \& Teodorovich (1980), with no specific name, and by Voisin (1991), as an 'internal potential'.

The independence of vertical vorticity and internal waves agrees fully with the discussion of Lighthill (1996). Written in the form

$$
\boldsymbol{u}=\left(\frac{\partial}{\partial t}-\nu \nabla^{2}\right) \frac{\partial}{\partial t} \nabla \chi-\boldsymbol{e}_{z} \times \nabla \psi-N^{2} \boldsymbol{e}_{z} \times\left(\boldsymbol{e}_{z} \times \nabla\right) \chi,
$$

the wave/vortex decomposition both confirms Hart's (1981) conjecture on the representation of the wavy component of the motion by a single scalar function, and corrects this conjecture by adding the vortical component overlooked by Hart because of the omission of any homogeneous solution of the system (2.1)-(2.3). The wave/vortex decomposition is also linked to the poloidal/toroidal decomposition, of the form $\nabla \times\left(A \boldsymbol{e}_{z}\right)+\nabla \times \nabla \times\left(B \boldsymbol{e}_{z}\right)$ with $A$ and $B$ scalar functions (see e.g. Holm \& Kimura 1991). This is confirmed by the expression of the vorticity

$$
\boldsymbol{\omega}=\boldsymbol{\nabla} \times \boldsymbol{u}=\nabla \times \nabla \times\left(\psi \boldsymbol{e}_{z}\right)-N^{2} \frac{\partial}{\partial z} \nabla \times\left(\chi \boldsymbol{e}_{z}\right) .
$$

A similar decomposition has indeed been used by Kistovich \& Chashechkin (2001a) for the velocity, but it is inadequate here as it requires the velocity field to be solenoidal everywhere, precluding the representation (2.2) of the forcing as a source of mass.

In the following, we shall investigate the waves exclusively, and omit the indices $\mathrm{w}$ accordingly.

\subsection{Green's functions}

A convenient starting point for the solution of the wave equation (2.9) is the inviscid Green's function $G(\boldsymbol{x}, t)$, which corresponds to impulsive point forcing and satisfies

$$
\left(\frac{\partial^{2}}{\partial t^{2}} \nabla^{2}+N^{2} \nabla_{\mathrm{h}}^{2}\right) G(\boldsymbol{x}, t)=\delta(\boldsymbol{x}) \delta(t)
$$

subject to the requirement of causality

$$
G(\boldsymbol{x}, t)=0 \text { for } t<0,
$$

that is of no disturbance prior to application of the forcing; here, $\delta(x)$ is the Dirac delta function. 
For monochromatic applications it is of interest to take Fourier transforms in time, defined for any function $f(\boldsymbol{x}, t)$ according to

$$
\begin{gathered}
f(\boldsymbol{k}, \omega)=\int f(\boldsymbol{x}, t) \exp [\mathrm{i}(\omega t-\boldsymbol{k} \cdot \boldsymbol{x})] \mathrm{d}^{3} x \mathrm{~d} t \\
f(\boldsymbol{x}, t)=\frac{1}{(2 \pi)^{4}} \int f(\boldsymbol{k}, \omega) \exp [\mathrm{i}(\boldsymbol{k} \cdot \boldsymbol{x}-\omega t)] \mathrm{d}^{3} k \mathrm{~d} \omega,
\end{gathered}
$$

and to consider not only the impulsive Green's function $G(\boldsymbol{x}, t)$, but also a monochromatic Green's function $G(\boldsymbol{x}, \omega)$ which satisfies

$$
\left[(\omega+\mathrm{i} 0)^{2} \nabla^{2}-N^{2} \nabla_{\mathrm{h}}^{2}\right] G(\boldsymbol{x}, \omega)=-\delta(\boldsymbol{x}) .
$$

Causality has become a requirement of analyticity in the upper half of the complex $\omega$-plane, a requirement met by replacing $\omega$ by $\omega+\mathrm{i} 0=\lim _{\epsilon \rightarrow 0}(\omega+\mathrm{i} \epsilon)$ with $\epsilon>0$, that is, by adding to the real frequency $\omega$ a positive imaginary part which is later allowed to tend to 0 ; alternatively, cuts may be inserted in the complex $\omega$-plane, extending from the real singularities of $G(\boldsymbol{x}, \omega)$ vertically downwards. Here the two procedures are equivalent, as only real frequencies $\omega$ will be considered.

The determination of the Green's functions was discussed by Voisin (1991), among others. Briefly, multiplying the horizontal coordinates by a factor $(\omega+\mathrm{i} 0) / N$ and the vertical coordinate by a factor $\left[(\omega+\mathrm{i} 0)^{2}-N^{2}\right]^{1 / 2} / N$ transforms $(2.16)$ into a Poisson equation, of solution

$$
G(\boldsymbol{x}, \omega)=\frac{1}{4 \pi\left[(\omega+\mathrm{i} 0)^{2}-N^{2}\right]^{1 / 2}\left[(\omega+\mathrm{i} 0)^{2} r^{2}-N^{2} z^{2}\right]^{1 / 2}},
$$

where $r=|\boldsymbol{x}|$. By Lighthill's (1958, chap. 4) rule, the singularities of this solution at the frequencies $\pm N|z| / r$ and $\pm N$ are responsible for the dominant asymptotic contributions to $G(\boldsymbol{x}, t)$ at large times $N|t| \gg 1$, yielding, by table 4 of Appendix A, a superposition of two terms

$$
G(\boldsymbol{x}, t) \sim-\frac{H(t)}{(2 \pi)^{3 / 2} N r_{\mathrm{h}}}\left[\frac{\cos (N t|z| / r-\pi / 4)}{(N t|z| / r)^{1 / 2}}+\frac{\sin (N t-\pi / 4)}{(N t)^{1 / 2}}\right],
$$

where $r_{\mathrm{h}}=\left|\boldsymbol{x}_{\mathrm{h}}\right|$ and $H(t)$ is the Heaviside step function. The first term represents gravity waves of frequency $N|z| / r$ conformable to the predictions of the group velocity theory, and the second term represents buoyancy oscillations at the frequency $N$.

For future use we also note the expression of the spatial transform

$$
G(\boldsymbol{k}, \omega)=\frac{1}{(\omega+\mathrm{i} 0)^{2} \kappa^{2}-N^{2} \kappa_{\mathrm{h}}^{2}},
$$

which follows immediately from (2.16). Here, $\kappa=|\boldsymbol{k}|$ and $\kappa_{\mathrm{h}}=\left|\boldsymbol{k}_{\mathrm{h}}\right|$, with $\boldsymbol{k}=(k, l, m)$ the wavenumber vector and $\boldsymbol{k}_{\mathrm{h}}=(k, l, 0)$ its horizontal projection.

\section{Inviscid steady point source}

The simplest localized monochromatic source is a steady point monopole $q(\boldsymbol{x}, t)=\mathscr{S} \delta(\boldsymbol{x}) \exp \left(-\mathrm{i} \omega_{0} t\right)$, oscillating with strength $\mathscr{S} \exp \left(-\mathrm{i} \omega_{0} t\right)$ at the frequency $0<\omega_{0}<N$ in an inviscid fluid. The wave equation for this source,

$$
\left(\frac{\partial^{2}}{\partial t^{2}} \nabla^{2}+N^{2} \nabla_{\mathrm{h}}^{2}\right) \chi(\boldsymbol{x}, t)=\mathscr{S} \delta(\boldsymbol{x}) \exp \left(-\mathrm{i} \omega_{0} t\right)
$$




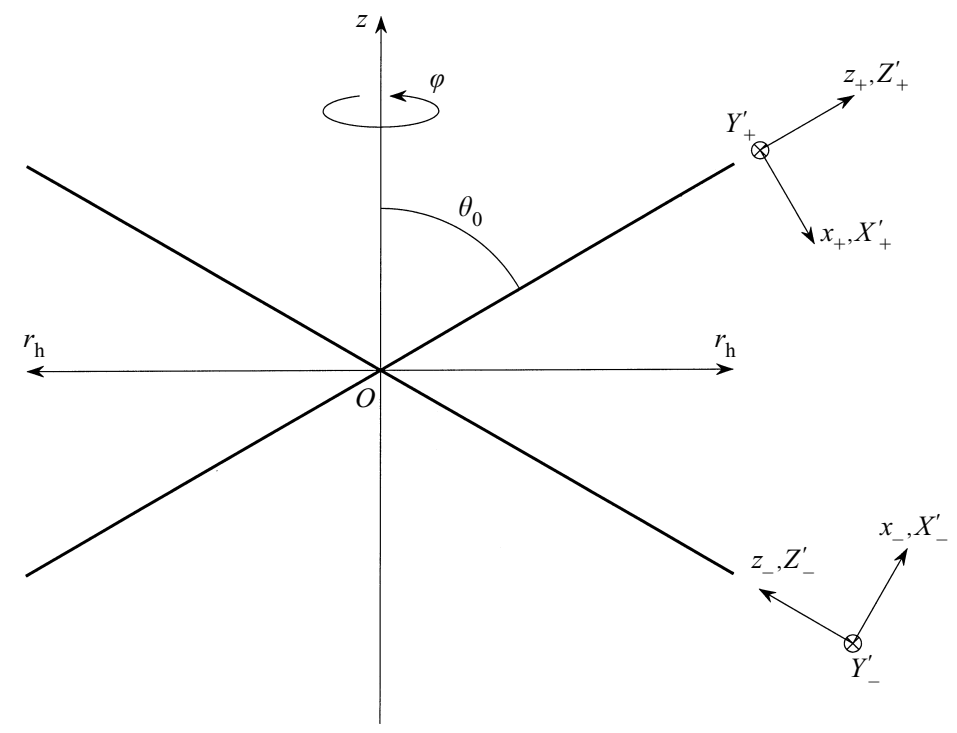

FIGURE 1. Geometry for internal wave radiation in real space. Waves of frequency $0<\omega_{0}<N$ propagate away from a point source at the origin $O$ on a double cone $\mathscr{C}_{0}$ of apex $O$, vertical axis and semi-angle $\theta_{0}=\arccos \left(\omega_{0} / N\right)$. Conical polar coordinates $\left(x_{ \pm}, \varphi, z_{ \pm}\right)$are introduced, such that in the upper half-space the energy propagates in the direction $O z_{+}$and the phase in the direction $O x_{+}$, while in the lower half-space the energy propagates opposite to the direction $O z_{-}$and the phase in the direction $O x_{-}$; the angle $\varphi$ is the polar angle in the horizontal plane. For an extended source, inside which $O$ is an arbitrarily chosen origin, in each vertical plane, of given $\varphi$, through $O$ a local system of Cartesian coordinates $\left(X_{ \pm}^{\prime}, Y_{ \pm}^{\prime}, Z_{ \pm}^{\prime}\right)$ is also introduced.

is solved immediately in terms of the monochromatic Green's function as

$$
\chi(\boldsymbol{x}, t)=\mathscr{S} G\left(\boldsymbol{x}, \omega_{0}\right) \exp \left(-\mathrm{i} \omega_{0} t\right)
$$

that is, by (2.17),

$$
\chi(\boldsymbol{x}, t)=-\mathrm{i} \frac{\mathscr{S}}{4 \pi N^{2} \sin \theta_{0}} \frac{\exp \left(-\mathrm{i} \omega_{0} t\right)}{\left(r_{\mathrm{h}}^{2} \cos ^{2} \theta_{0}-z^{2} \sin ^{2} \theta_{0}+\mathrm{i} 0\right)^{1 / 2}},
$$

where $\theta_{0}=\arccos \left(\omega_{0} / N\right)$.

The singularity of this solution on the characteristic double cone $\mathscr{C}_{0}$ of vertical axis, apex at the source and semi-angle $\theta_{0}$ dictates the introduction of two systems of conical polar coordinates $\left(x_{+}, \varphi, z_{+}\right)$and $\left(x_{-}, \varphi, z_{-}\right)$, represented in figure 1 , defined in cylindrical coordinates $\left(r_{\mathrm{h}}, \varphi, z\right)$ by

$$
r_{\mathrm{h}}=x_{ \pm} \cos \theta_{0} \pm z_{ \pm} \sin \theta_{0}, \quad z=\mp x_{ \pm} \sin \theta_{0}+z_{ \pm} \cos \theta_{0},
$$

and such that $x_{+}=0$ on the upper cone and $x_{-}=0$ on the lower cone; the respective unit vectors are denoted as $\left(\boldsymbol{e}_{x_{+}}, \boldsymbol{e}_{\varphi}, \boldsymbol{e}_{z_{+}}\right)$and $\left(\boldsymbol{e}_{x_{-}}, \boldsymbol{e}_{\varphi}, \boldsymbol{e}_{z_{-}}\right)$. In terms of the characteristic coordinates $\left(x_{+}, x_{-}\right)$alone, introduced by Hurley (1969), we have

$$
x_{ \pm}=r_{\mathrm{h}} \cos \theta_{0} \mp z \sin \theta_{0},
$$

and reciprocally

$$
r_{\mathrm{h}}=\frac{x_{+}+x_{-}}{2 \cos \theta_{0}}, \quad z=\frac{x_{-}-x_{+}}{2 \sin \theta_{0}}
$$


The wave function becomes

$$
\chi(\boldsymbol{x}, t)=-\mathrm{i} \frac{\mathscr{S}}{4 \pi N^{2} \sin \theta_{0}} \frac{\exp \left(-\mathrm{i} \omega_{0} t\right)}{\left(x_{+} x_{-}+\mathrm{i} 0\right)^{1 / 2}},
$$

which may be factorized as

$$
\chi(\boldsymbol{x}, t)=-\mathrm{i} \frac{\mathscr{S}}{4 \pi N^{2} \sin \theta_{0}} \frac{\exp \left(-\mathrm{i} \omega_{0} t\right)}{\left(x_{+}+\mathrm{i} 0 \operatorname{sign} z\right)^{1 / 2}\left(x_{-}-\mathrm{i} 0 \operatorname{sign} z\right)^{1 / 2}} .
$$

The associated pressure and velocity are, by (2.11),

$$
\begin{gathered}
p(\boldsymbol{x}, t)=\frac{\rho_{0} N \mathscr{S} \cos \theta_{0} \sin \theta_{0}}{4 \pi} \frac{\exp \left(-\mathrm{i} \omega_{0} t\right)}{\left(x_{+} x_{-}+\mathrm{i} 0\right)^{1 / 2}}, \\
\boldsymbol{u}(\boldsymbol{x}, t)=\mathrm{i} \frac{\mathscr{S} \cos \theta_{0}}{8 \pi} \frac{x_{-} \boldsymbol{e}_{z_{+}}-x_{+} \boldsymbol{e}_{z_{-}}}{\left(x_{+} x_{-}+\mathrm{i} 0\right)^{3 / 2}} \exp \left(-\mathrm{i} \omega_{0} t\right) .
\end{gathered}
$$

They are everywhere $\pi / 2$ out of phase, implying no energy radiation, except on the double cone $\mathscr{C}_{0}$ where their amplitudes diverge and their phases jump, by $\pi / 2$ for the pressure and $3 \pi / 2$ for the velocity. Hence, consistently with the predictions of the group velocity theory, recalled in $\S 1$, calculations based on the model of an inviscid steady point source are able to predict where the waves are found - on $\mathscr{C}_{0}$ - but fail to describe them there. The rest of this paper will propose amendments to the theory, so as to provide the missing description.

\section{Waves from an extended source}

We consider a steady monochromatic source $q(\boldsymbol{x}, t)=q_{0}(\boldsymbol{x}) \exp \left(-\mathrm{i} \omega_{0} t\right)$, of finite radius $a$ in the sense that $q_{0}(\boldsymbol{x})$ vanishes rapidly for $r \gg a$, oscillating at the frequency $0<\omega_{0}<N$ in an inviscid fluid. The wave equation for this source,

$$
\left(\frac{\partial^{2}}{\partial t^{2}} \nabla^{2}+N^{2} \nabla_{\mathrm{h}}^{2}\right) \chi(\boldsymbol{x}, t)=q_{0}(\boldsymbol{x}) \exp \left(-\mathrm{i} \omega_{0} t\right)
$$

will be solved first in real space then in Fourier space, in sequence, for reasons that will arise in the course of the calculation.

\subsection{The real-space way}

In real space, the solution to (4.1) follows from the convolution of the source term with the monochromatic Green's function, yielding

$$
\chi(\boldsymbol{x}, t)=\exp \left(-\mathrm{i} \omega_{0} t\right) \int q_{0}\left(\boldsymbol{x}^{\prime}\right) G\left(\boldsymbol{x}-\boldsymbol{x}^{\prime}, \omega_{0}\right) \mathrm{d}^{3} x^{\prime},
$$

that is, by (2.17),

where

$$
\chi(\boldsymbol{x}, t)=-\mathrm{i} \frac{\exp \left(-\mathrm{i} \omega_{0} t\right)}{4 \pi N^{2} \sin \theta_{0}} \int \frac{q_{0}\left(\boldsymbol{x}^{\prime}\right)}{D\left(\boldsymbol{x}-\boldsymbol{x}^{\prime}\right)} \mathrm{d}^{3} x^{\prime}
$$

$$
D\left(\boldsymbol{x}-\boldsymbol{x}^{\prime}\right)=\left[\left(\boldsymbol{x}_{\mathrm{h}}-\boldsymbol{x}_{\mathrm{h}}^{\prime}\right)^{2} \cos ^{2} \theta_{0}-\left(z-z^{\prime}\right)^{2} \sin ^{2} \theta_{0}+\mathrm{i} 0\right]^{1 / 2} \text {. }
$$

This convolution product cannot be effected exactly. To overcome the difficulty we proceed in two stages.

First, an arbitrary origin $O$ being chosen inside the source, the fixed observation location $\boldsymbol{x}$ is expressed in conical polar coordinates $\left(x_{ \pm}, \varphi, z_{ \pm}\right)$, and the variable emission point $\boldsymbol{x}^{\prime}$ is expressed in local Cartesian coordinates $\left(x_{x_{ \pm}}^{\prime}, x_{\varphi}^{\prime}, x_{z_{ \pm}}^{\prime}\right)$, namely by 
its components along the unit vectors $\left(\boldsymbol{e}_{x_{ \pm}}, \boldsymbol{e}_{\varphi}, \boldsymbol{e}_{z_{ \pm}}\right)$of the conical polar coordinate system associated with $\boldsymbol{x}$. For clarity, these coordinates $\left(x_{x_{+}}^{\prime}, x_{\varphi}^{\prime}, x_{z_{+}}^{\prime}\right)$, represented in figure 1 , are denoted as $\left(X_{ \pm}^{\prime}, Y_{ \pm}^{\prime}, Z_{ \pm}^{\prime}\right)$ so that

$$
\begin{gathered}
x^{\prime}=X_{ \pm}^{\prime} \cos \theta_{0} \cos \varphi-Y_{ \pm}^{\prime} \sin \varphi \pm Z_{ \pm}^{\prime} \sin \theta_{0} \cos \varphi, \\
y^{\prime}=X_{ \pm}^{\prime} \cos \theta_{0} \sin \varphi+Y_{ \pm}^{\prime} \cos \varphi \pm Z_{ \pm}^{\prime} \sin \theta_{0} \sin \varphi, \\
z^{\prime}=\mp X_{ \pm}^{\prime} \sin \theta_{0}+Z_{ \pm}^{\prime} \cos \theta_{0} .
\end{gathered}
$$

The observation location becomes $\left(X_{ \pm}=x_{ \pm}, Y_{ \pm}=0, Z_{ \pm}=z_{ \pm}\right)$, and the denominator (4.4) becomes

$$
D\left(\boldsymbol{x}-\boldsymbol{x}^{\prime}\right)=\left[\left(x_{+}-X_{+}^{\prime}\right)\left(x_{-}-X_{-}^{\prime}\right)+Y_{ \pm}^{\prime 2} \cos ^{2} \theta_{0}+\mathrm{i} 0\right]^{1 / 2} .
$$

Secondly, the far-field approximation $r \gg a$ is introduced, such that $r^{\prime} / r=O(a / r)$. The obvious expansion of (4.6), namely

$$
D\left(\boldsymbol{x}-\boldsymbol{x}^{\prime}\right) \sim\left(x_{+} x_{-}-x_{-} X_{+}^{\prime}-x_{+} X_{-}^{\prime}+\mathrm{i} 0\right)^{1 / 2},
$$

breaks down in the vicinity of the double cone $\mathscr{C}_{0}$ where the zeroth- and firstorder terms become equally important. Space must, accordingly, be divided into two zones, depending on whether the angular separation from $\mathscr{C}_{0}$ is finite and $O(1)$, or infinitesimal and $O(a / r)$, respectively, as $r / a \rightarrow \infty$ (a point first made by Crighton 1986). To express the separation analytically we use the coordinates $\left(x_{+}, \varphi, z_{+}\right)$ and $\left(X_{+}^{\prime}, Y_{+}^{\prime}, Z_{+}^{\prime}\right)$ in the upper half-space $z>0$, and the coordinates $\left(x_{-}, \varphi, z_{-}\right)$and $\left(X_{-}^{\prime}, Y_{-}^{\prime}, Z_{-}^{\prime}\right)$ in the lower half-space $z<0$. This choice will hereinafter be denoted by an index \pm , with the convention $\pm=\operatorname{sign} z$.

In the outer zone, corresponding to $\left|z_{ \pm}\right| / a \rightarrow \infty$ with $\left|x_{ \pm}\right| /\left|z_{ \pm}\right|=O(1)$, we have

$$
D\left(\boldsymbol{x}-\boldsymbol{x}^{\prime}\right) \sim\left(x_{+} x_{-}+\mathrm{i} 0\right)^{1 / 2},
$$

from which the wave function (3.7) is recovered with $\mathscr{S}=\int q_{0}\left(\boldsymbol{x}^{\prime}\right) \mathrm{d}^{3} x^{\prime}$.

In the inner zone, corresponding to $\left|z_{ \pm}\right| / a \rightarrow \infty$ with $\left|x_{ \pm}\right| / a=O(1)$, we have instead

$$
D\left(\boldsymbol{x}-\boldsymbol{x}^{\prime}\right) \sim x_{\overline{+}}^{1 / 2}\left(x_{ \pm}-X_{ \pm}^{\prime}+\mathrm{i} 0\right)^{1 / 2} \sim\left|z_{ \pm}\right|^{1 / 2} \sin ^{1 / 2}\left(2 \theta_{0}\right)\left(x_{ \pm}-X_{ \pm}^{\prime}+\mathrm{i} 0\right)^{1 / 2},
$$

so that

$$
\chi(\boldsymbol{x}, t) \sim-\mathrm{i} \frac{\exp \left(-\mathrm{i} \omega_{0} t\right)}{2^{5 / 2} \pi N^{2}\left|z_{ \pm}\right|^{1 / 2} \cos ^{1 / 2} \theta_{0} \sin ^{3 / 2} \theta_{0}} \int \frac{Q_{ \pm}\left(\boldsymbol{X}_{ \pm}^{\prime} \mid \varphi\right)}{\left(x_{ \pm}-X_{ \pm}^{\prime}+\mathrm{i} 0\right)^{1 / 2}} \mathrm{~d}^{3} X_{ \pm}^{\prime},
$$

where the new source function $Q_{ \pm}\left(\boldsymbol{X}_{ \pm}^{\prime} \mid \varphi\right)=q_{0}\left(\boldsymbol{x}^{\prime}\right)$, with $\boldsymbol{X}_{ \pm}^{\prime}=\left(X_{ \pm}^{\prime}, Y_{ \pm}^{\prime}, Z_{ \pm}^{\prime}\right)$, depends on the polar angle $\varphi$ through (4.5). Indeed, at a fixed emission location $\boldsymbol{x}^{\prime}$ whose local coordinates $\left(X_{ \pm}^{\prime}, Y_{ \pm}^{\prime}, Z_{ \pm}^{\prime}\right)$ vary as a consequence of a variation of the observation angle $\varphi$, the source function satisfies

$$
\frac{\partial Q_{ \pm}}{\partial \varphi}=\left(X_{ \pm}^{\prime} \cos \theta_{0} \pm Z_{ \pm}^{\prime} \sin \theta_{0}\right) \frac{\partial Q_{ \pm}}{\partial Y_{ \pm}^{\prime}}-Y_{ \pm}^{\prime}\left(\cos \theta_{0} \frac{\partial Q_{ \pm}}{\partial X_{ \pm}^{\prime}} \pm \sin \theta_{0} \frac{\partial Q_{ \pm}}{\partial Z_{ \pm}^{\prime}}\right) .
$$

The one-dimensional convolution product (4.10), simpler than the original threedimensional product (4.3), is simplified further by introducing spatial Fourier transforms and by using the convolution theorem together with the transform

$$
\int \frac{\exp \left(-\mathrm{i} \boldsymbol{K}_{ \pm} \cdot \boldsymbol{X}_{ \pm}^{\prime}\right)}{\left(X_{ \pm}^{\prime}+\mathrm{i} 0\right)^{1 / 2}} \mathrm{~d}^{3} X_{ \pm}^{\prime}=8 \pi^{5 / 2} \exp \left(-\mathrm{i} \frac{1}{4} \pi\right) \frac{H\left(K_{ \pm}\right)}{K_{ \pm}^{1 / 2}} \delta\left(L_{ \pm}\right) \delta\left(M_{ \pm}\right)
$$




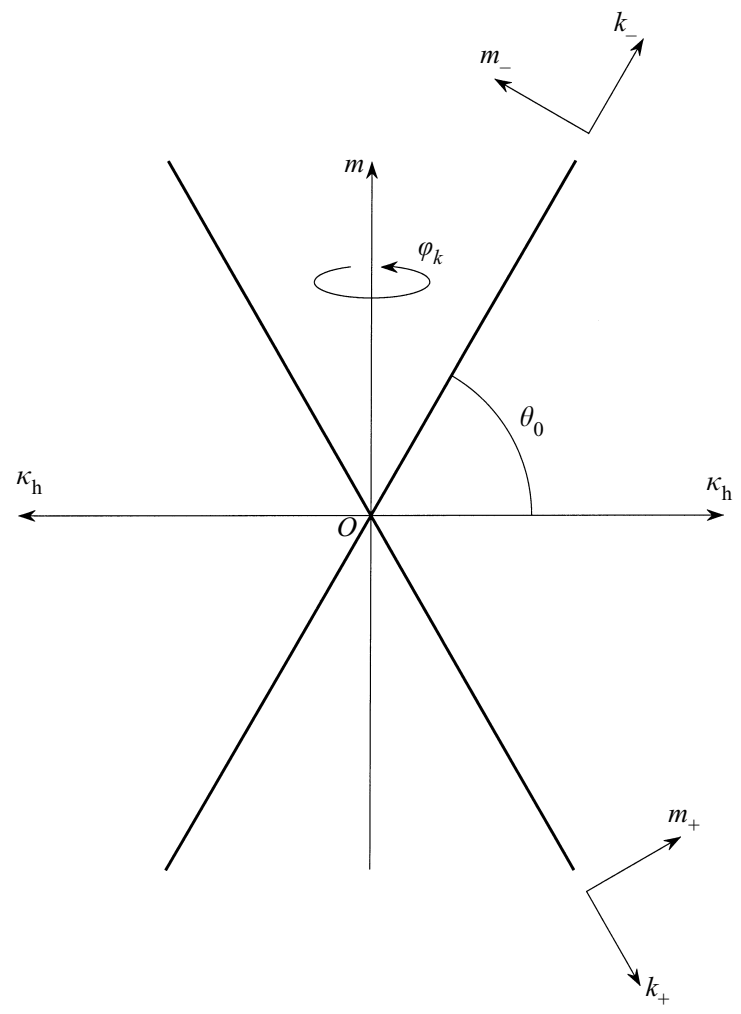

FIGURE 2. Geometry for internal wave radiation in Fourier space. At the frequency $0<\omega_{0}<N$, the wavenumber vectors are situated on a double cone $\mathscr{C}_{0}^{(k)}$ of apex the origin $O$, vertical axis and semi-angle $\pi / 2-\theta_{0}=\arcsin \left(\omega_{0} / N\right)$. In conical polar coordinates $\left(k_{ \pm}, \varphi_{k}, m_{ \pm}\right)$, in the upper half-space the wavenumber vectors point in the direction $O k_{-}$and the group velocity vectors opposite to the direction $\mathrm{Om}_{-}$, while in the lower half-space the wavenumber vectors point in the direction $\mathrm{Ok}_{+}$and the group velocity vectors in the direction $\mathrm{Om}_{+}$.

deduced from table 4. The wavenumber vector $\boldsymbol{k}$ is expressed in local Cartesian coordinates $\left(K_{ \pm}, L_{ \pm}, M_{ \pm}\right)$, conjugate to $\left(X_{ \pm}^{\prime}, Y_{ \pm}^{\prime}, Z_{ \pm}^{\prime}\right)$ and defined by

$$
\begin{gathered}
k=K_{ \pm} \cos \theta_{0} \cos \varphi-L_{ \pm} \sin \varphi \pm M_{ \pm} \sin \theta_{0} \cos \varphi, \\
l=K_{ \pm} \cos \theta_{0} \sin \varphi+L_{ \pm} \cos \varphi \pm M_{ \pm} \sin \theta_{0} \sin \varphi, \\
m=\mp K_{ \pm} \sin \theta_{0}+M_{ \pm} \cos \theta_{0},
\end{gathered}
$$

with $\boldsymbol{K}_{ \pm}=\left(K_{ \pm}, L_{ \pm}, M_{ \pm}\right)$. The wave function becomes

$$
\chi(\boldsymbol{x}, t) \sim \frac{\exp \left[-\mathrm{i}\left(\omega_{0} t+3 \pi / 4\right)\right]}{2^{5 / 2} \pi^{3 / 2} N^{2}\left|z_{ \pm}\right|^{1 / 2} \cos ^{1 / 2} \theta_{0} \sin ^{3 / 2} \theta_{0}} \int_{0}^{\infty} Q_{ \pm}\left(K_{ \pm}, 0,0 \mid \varphi\right) \exp \left(\mathrm{i} K_{ \pm} x_{ \pm}\right) \frac{\mathrm{d} K_{ \pm}}{K_{ \pm}^{1 / 2}},
$$

where $Q_{ \pm}\left(\boldsymbol{K}_{ \pm} \mid \varphi\right)=q_{0}(\boldsymbol{k})$.

This result is identical, after appropriate changes of notation and variables, to that (350) of Lighthill $(1978, \S 4.10)$. It involves wavenumber vectors situated on the wavenumber surface, a double cone $\mathscr{C}_{0}^{(k)}$ of apex $O$, vertical axis and semi-angle $\pi / 2-\theta_{0}=\arcsin \left(\omega_{0} / N\right)$, defined by the dispersion relation $\omega_{0}^{2} \kappa^{2}-N^{2} \kappa_{\mathrm{h}}^{2}=0$ and represented in figure 2. Specifically, (4.14) involves the section of $\mathscr{C}_{0}^{(k)}$ by the vertical 
plane through $O$ containing the observer, a plane of given polar angle $\varphi_{k}=\varphi$, such that the associated group velocity vectors point toward the observer. Accordingly, the conical polar coordinates $\left(k_{ \pm}, \varphi_{k}, m_{ \pm}\right)$, defined in cylindrical coordinates $\left(\kappa_{\mathrm{h}}, \varphi_{k}, m\right)$ by

$$
\kappa_{\mathrm{h}}=k_{ \pm} \cos \theta_{0} \pm m_{ \pm} \sin \theta_{0}, \quad m=\mp k_{ \pm} \sin \theta_{0}+m_{ \pm} \cos \theta_{0},
$$

are especially appropriate for expressing the wave function in a way that avoids resorting to local Cartesian coordinates. We obtain

$$
\chi(x, t) \sim \frac{\exp \left[-\mathrm{i}\left(\omega_{0} t+3 \pi / 4\right)\right]}{2^{5 / 2} \pi^{3 / 2} N^{2}\left|z_{ \pm}\right|^{1 / 2} \cos ^{1 / 2} \theta_{0} \sin ^{3 / 2} \theta_{0}} \int_{0}^{\infty} q_{ \pm}\left(k_{ \pm}, \varphi, 0\right) \exp \left(\mathrm{i} k_{ \pm} x_{ \pm}\right) \frac{\mathrm{d} k_{ \pm}}{k_{ \pm}^{1 / 2}},
$$

where $q_{ \pm}\left(k_{ \pm}, \varphi_{k}, m_{ \pm}\right)=q_{0}(\boldsymbol{k})$.

\subsection{The Fourier-space way}

The final expression of the wave function as a Fourier integral, and the identity of this integral to Lighthill's $(1978, \S 4.10)$ result, obtained directly in Fourier space, suggests an additional investigation of the wave equation (4.1) by Fourier transformation. The wave function is written as the inverse transform

$$
\chi(\boldsymbol{x}, t)=\frac{\exp \left(-\mathrm{i} \omega_{0} t\right)}{8 \pi^{3}} \int q_{0}(\boldsymbol{k}) G\left(\boldsymbol{k}, \omega_{0}\right) \exp (\mathrm{i} \boldsymbol{k} \cdot \boldsymbol{x}) \mathrm{d}^{3} k,
$$

that is, by (2.19),

$$
\chi(\boldsymbol{x}, t)=\frac{\exp \left(-\mathrm{i} \omega_{0} t\right)}{8 \pi^{3}} \int \frac{q_{0}(\boldsymbol{k}) \exp (\mathrm{i} \boldsymbol{k} \cdot \boldsymbol{x})}{\omega_{0}^{2} \kappa^{2}-N^{2} \kappa_{\mathrm{h}}^{2}+\mathrm{i} 0} \mathrm{~d}^{3} k,
$$

an integral of integrand singular on the wavenumber surface $\mathscr{C}_{0}^{(k)}$. Lighthill (1978, $\S 4.10)$ proceeds in local Cartesian coordinates $\left(K_{ \pm}, L_{ \pm}, M_{ \pm}\right)$, using $\left(K_{+}, L_{+}, M_{+}\right)$for evaluating the contribution of the lower half-space $m<0$, and $\left(K_{-}, L_{-}, M_{-}\right)$for evaluating the contribution of the upper half-space $m>0$, so that $\pm=-\operatorname{sign} m$. (In this discussion the convention $\pm=\operatorname{sign} z$ of the previous section does not apply.) The calculation involves two stages: (i) the residue theorem is applied to the integral with respect to $M_{ \pm}$, after deformation of contour in the complex $M_{ \pm}$-plane; (ii) the stationary phase method is applied to the integral with respect to $L_{ \pm}$, on the far-field assumption $r \rightarrow \infty$. However, one point that Lighthill overlooked is that, in each halfspace $m \lessgtr 0$, the path of integration with respect to $M_{ \pm}$is a half-line $\pm M_{ \pm}>K_{ \pm} \tan \theta_{0}$. Application of the residue theorem in the complex $M_{ \pm}$-plane is then complicated by the addition, to the contribution of the real pole of the integrand, of an integral along a path parallel to the imaginary axis. Actually, Lighthill (1990) produced later, in another context, an emendation to his theory, acknowledging the necessity of considering the complex continuation of the real wavenumber surface. A second objection lies in the application of the stationary phase method to the integral with respect to $L_{ \pm}$, regardless of the remaining integration variable $K_{ \pm}$, whereas this method is not applicable when $K_{ \pm}$is of order $1 / r$.

We proceed instead in cylindrical coordinates $\left(\kappa_{\mathrm{h}}, \varphi_{k}, m\right)$. The wave function (4.18) becomes

$$
\chi(x, t)=\frac{\exp \left(-\mathrm{i} \omega_{0} t\right)}{8 \pi^{3} N^{2}} \int_{0}^{\infty} \kappa_{\mathrm{h}} \mathrm{d} \kappa_{\mathrm{h}} \int_{0}^{2 \pi} \mathrm{d} \varphi_{k} \int_{-\infty}^{\infty} \mathrm{d} m q_{0}(\boldsymbol{k}) \frac{\exp \left\{\mathrm{i}\left[\kappa_{\mathrm{h}} r_{\mathrm{h}} \cos \left(\varphi_{k}-\varphi\right)+m z\right]\right\}}{m^{2} \cos ^{2} \theta_{0}-\kappa_{\mathrm{h}}^{2} \sin ^{2} \theta_{0}+\mathrm{i} 0} .
$$




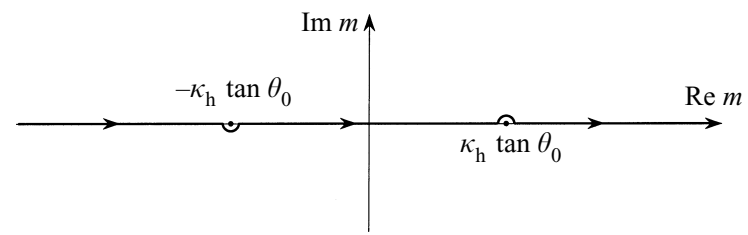

Figure 3. Causal integration path in the complex $m$-plane.

In the complex $m$-plane the integrand has two simple poles, at $\kappa_{\mathrm{h}} \tan \theta_{0}-\mathrm{i} 0$ and $-\kappa_{\mathrm{h}} \tan \theta_{0}+\mathrm{i} 0$, respectively, corresponding to the path of integration shown in figure 3 . Deformation of this path selects the pole

$$
m=-\kappa_{\mathrm{h}} \tan \theta_{0} \operatorname{sign} z,
$$

that is $m_{ \pm}=0$ in conical polar coordinates $\left(k_{ \pm}, \varphi_{k}, m_{ \pm}\right)$, with again $\pm=\operatorname{sign} z$. The residue at this pole is

$$
\chi(\boldsymbol{x}, t)=-\mathrm{i} \frac{\exp \left(-\mathrm{i} \omega_{0} t\right)}{8 \pi^{2} N^{2} \sin \theta_{0}} \int_{0}^{\infty} \mathrm{d} k_{ \pm} \int_{0}^{2 \pi} \mathrm{d} \varphi_{k} q_{ \pm}\left(k_{ \pm}, \varphi_{k}, 0\right) \exp \left[\mathrm{i} k_{ \pm} \Phi\left(\varphi_{k} \mid \boldsymbol{x}\right)\right],
$$

where

$$
\begin{aligned}
\Phi\left(\varphi_{k} \mid \boldsymbol{x}\right) & =r_{\mathrm{h}} \cos \theta_{0} \cos \left(\varphi_{k}-\varphi\right)-|z| \sin \theta_{0} \\
& =x_{ \pm} \cos ^{2}\left(\frac{\varphi_{k}-\varphi}{2}\right)-x_{\mp} \sin ^{2}\left(\frac{\varphi_{k}-\varphi}{2}\right) .
\end{aligned}
$$

This expression is exact and does not involve any far-field approximation: to the contribution (4.16) of the section $\varphi_{k}=\varphi$ of the wavenumber surface $\mathscr{C}_{0}^{(k)}$, a section composed of wavenumber vectors such that the associated group velocity vectors point toward the observer, (4.21) adds the contribution of every other section $\varphi_{k} \neq \varphi$, imposing only by (4.20) the restriction that the group velocity vectors have vertical components oriented toward the observer while the horizontal components may point in any direction.

We investigate now the far field $r \gg a$. With $q_{0}(\boldsymbol{x})$ varying on a scale $a$, its transform $q_{0}(\boldsymbol{k})$ varies on a scale $1 / a$. Accordingly, the expansion of the wave function (4.21) combines two asymptotic contributions: one, local, from the wavenumbers $k_{ \pm}$of order $1 / r$, such that $k_{ \pm} a \ll 1$ and thus $q_{ \pm}\left(k_{ \pm}, \varphi_{k}, 0\right) \sim q_{0}(\boldsymbol{k}=\mathbf{0})=\mathscr{S}$; and the other, global, from the wavenumbers $k_{ \pm}$of order $1 / a$, such that $k_{ \pm} r \gg 1$ thus allowing the application of the method of stationary phase to the integral with respect to $\varphi_{k}$. The two contributions are separated as discussed by Hinch $(1991, \S 3.4)$, by splitting the range of integration into two at an intermediate scale $1 / d$ such that $a \ll d \ll r$.

The local contribution $\chi_{1}(\boldsymbol{x}, t)$ of the small range $0<k_{ \pm}<1 / d$ is first simplified by writing $q_{ \pm}\left(k_{ \pm}, \varphi_{k}, 0\right) \sim \mathscr{S}$, then separated into a difference of two terms

$$
\chi_{1}(\boldsymbol{x}, t) \sim-\mathrm{i} \frac{\mathscr{S}}{8 \pi^{2} N^{2} \sin \theta_{0}} \exp \left(-\mathrm{i} \omega_{0} t\right)\left(\int_{0}^{\infty}-\int_{1 / d}^{\infty}\right) \mathrm{d} k_{ \pm} \int_{0}^{2 \pi} \mathrm{d} \varphi_{k} \exp \left[\mathrm{i} k_{ \pm} \Phi\left(\varphi_{k} \mid \boldsymbol{x}\right)\right] .
$$

The first term, corresponding to $0<k_{ \pm}<\infty$, is calculated by applying table 5 of Appendix A to the integral with respect to $k_{ \pm}$, and the residue theorem to the integral 
with respect to $\varphi_{k}$, to obtain

$$
\int_{0}^{2 \pi} \mathrm{d} \varphi_{k} \int_{0}^{\infty} \mathrm{d} k_{ \pm} \exp \left[\mathrm{i} k_{ \pm} \Phi\left(\varphi_{k} \mid \boldsymbol{x}\right)\right]=\mathrm{i} \int_{0}^{2 \pi} \frac{\mathrm{d} \varphi_{k}}{\Phi\left(\varphi_{k} \mid \boldsymbol{x}\right)+\mathrm{i} 0}=\frac{2 \pi}{\left(x_{+} x_{-}+\mathrm{i} 0\right)^{1 / 2}},
$$

where the pole of the integrand is associated with an angle $\varphi_{k}$ such that

$$
\tan \left(\frac{\varphi_{k}-\varphi}{2}\right)=\left(\frac{x_{ \pm}+\mathrm{i} 0}{x_{\mp}}\right)^{1 / 2} .
$$

For $x_{ \pm}<0$ this angle is imaginary, consistent with the above discussion about the necessity to consider a complex wavenumber surface. The second term, corresponding to $1 / d<k_{ \pm}<\infty$, is calculated by applying the method of stationary phase to the integral with respect to $\varphi_{k}$, with stationary points $\varphi_{k}=\varphi$ and $\varphi+\pi$, and table 5 to the integral with respect to $k_{ \pm}$. The local contribution becomes

$$
\begin{aligned}
\chi_{1}(\boldsymbol{x}, t) \sim- & \mathrm{i} \frac{\mathscr{S}}{4 \pi N^{2} \sin \theta_{0}} \frac{\exp \left(-\mathrm{i} \omega_{0} t\right)}{\left(x_{+} x_{-}+\mathrm{i} 0\right)^{1 / 2}} \\
& +\mathrm{i} \frac{\mathscr{S}}{4 \pi^{3 / 2} N^{2} \sin \theta_{0}} \frac{\exp \left(-\mathrm{i} \omega_{0} t\right)}{\left(x_{+}+x_{-}\right)^{1 / 2}} \frac{\Gamma\left[\frac{1}{2},-\mathrm{i}\left(x_{ \pm}+\mathrm{i} 0\right) / d\right]}{\left(x_{ \pm}+\mathrm{i} 0\right)^{1 / 2}} \\
& +\mathrm{i} \frac{\mathscr{S}}{4 \pi^{3 / 2} N^{2} \sin \theta_{0}} \frac{\exp \left(-\mathrm{i} \omega_{0} t\right)}{\left(x_{+}+x_{-}\right)^{1 / 2}} \frac{\Gamma\left[\frac{1}{2}, \mathrm{i}\left(x_{\mp}-\mathrm{i} 0\right) / d\right]}{\left(x_{\mp}-\mathrm{i} 0\right)^{1 / 2}},
\end{aligned}
$$

with $\Gamma(\mu, x)$ the complementary incomplete gamma function.

The global contribution $\chi_{\mathrm{g}}(\boldsymbol{x}, t)$ of the remainder of the range $1 / d<k_{ \pm}<\infty$ is first simplified by applying the method of stationary phase to the integral with respect to $\varphi_{k}$, then separated into a difference of two terms

$$
\begin{aligned}
\chi_{\mathrm{g}}(\boldsymbol{x}, t) \sim & \frac{1}{4 \pi^{3 / 2} N^{2} \sin \theta_{0}} \frac{\exp \left[-\mathrm{i}\left(\omega_{0} t+3 \pi / 4\right)\right]}{\left(x_{+}+x_{-}\right)^{1 / 2}}\left(\int_{0}^{\infty}-\int_{0}^{1 / d}\right) \frac{\mathrm{d} k_{ \pm}}{k_{ \pm}^{1 / 2}} \\
& \times\left[q_{ \pm}\left(k_{ \pm}, \varphi, 0\right) \exp \left(\mathrm{i} k_{ \pm} x_{ \pm}\right)+\mathrm{i} q_{ \pm}\left(k_{ \pm}, \varphi+\pi, 0\right) \exp \left(-\mathrm{i} k_{ \pm} x_{\mp}\right)\right] .
\end{aligned}
$$

The second term, corresponding to $0<k_{ \pm}<1 / d$, is calculated by writing $q_{ \pm}\left(k_{ \pm}, \varphi_{k}, 0\right) \sim \mathscr{S}$ and applying table 5 . The global contribution becomes

$$
\begin{aligned}
\chi_{\mathrm{g}}(\boldsymbol{x}, t) \sim & \frac{1}{4 \pi^{3 / 2} N^{2} \sin \theta_{0}} \frac{\exp \left[-\mathrm{i}\left(\omega_{0} t+3 \pi / 4\right)\right]}{\left(x_{+}+x_{-}\right)^{1 / 2}} \int_{0}^{\infty} q_{ \pm}\left(k_{ \pm}, \varphi, 0\right) \exp \left(\mathrm{i} k_{ \pm} x_{ \pm}\right) \frac{\mathrm{d} k_{ \pm}}{k_{ \pm}^{1 / 2}} \\
& +\frac{1}{4 \pi^{3 / 2} N^{2} \sin \theta_{0}} \frac{\exp \left[-\mathrm{i}\left(\omega_{0} t+\pi / 4\right)\right]}{\left(x_{+}+x_{-}\right)^{1 / 2}} \int_{0}^{\infty} q_{ \pm}\left(k_{ \pm}, \varphi+\pi, 0\right) \exp \left(-\mathrm{i} k_{ \pm} x_{\mp}\right) \frac{\mathrm{d} k_{ \pm}}{k_{ \pm}^{1 / 2}} \\
& +\mathrm{i} \frac{\mathscr{S}}{4 \pi^{3 / 2} N^{2} \sin \theta_{0}} \frac{\exp \left(-\mathrm{i} \omega_{0} t\right)}{\left(x_{+}+x_{-}\right)^{1 / 2}} \frac{\gamma\left(\frac{1}{2},-\mathrm{i} x_{ \pm} / d\right)}{x_{ \pm}^{1 / 2}} \\
& +\mathrm{i} \frac{\mathscr{S}}{4 \pi^{3 / 2} N^{2} \sin \theta_{0}} \frac{\exp \left(-\mathrm{i} \omega_{0} t\right)}{\left(x_{+}+x_{-}\right)^{1 / 2}} \frac{\gamma\left(\frac{1}{2}, \mathrm{i} x_{\mp} / d\right)}{x_{\mp}^{1 / 2}}
\end{aligned}
$$

with $\gamma(\mu, x)$ the incomplete gamma function, such that $\gamma(\mu, x)+\Gamma(\mu, x)=\Gamma(\mu)$. 
The combination of the two contributions is independent of the artificially introduced $d$, and given by

$$
\begin{aligned}
\chi(\boldsymbol{x}, t) \sim & \frac{1}{4 \pi^{3 / 2} N^{2} \sin \theta_{0}} \frac{\exp \left[-\mathrm{i}\left(\omega_{0} t+3 \pi / 4\right)\right]}{\left(x_{+}+x_{-}\right)^{1 / 2}} \int_{0}^{\infty} q_{ \pm}\left(k_{ \pm}, \varphi, 0\right) \exp \left(\mathrm{i} k_{ \pm} x_{ \pm}\right) \frac{\mathrm{d} k_{ \pm}}{k_{ \pm}^{1 / 2}} \\
& +\mathrm{i} \frac{\mathscr{S}}{4 \pi N^{2} \sin \theta_{0}} \frac{\exp \left(-\mathrm{i} \omega_{0} t\right)}{\left(x_{+}+x_{-}\right)^{1 / 2}\left(x_{ \pm}+\mathrm{i} 0\right)^{1 / 2}} \\
& +\frac{1}{4 \pi^{3 / 2} N^{2} \sin \theta_{0}} \frac{\exp \left[-\mathrm{i}\left(\omega_{0} t+\pi / 4\right)\right]}{\left(x_{+}+x_{-}\right)^{1 / 2}} \int_{0}^{\infty} q_{ \pm}\left(k_{ \pm}, \varphi+\pi, 0\right) \exp \left(-\mathrm{i} k_{ \pm} x_{\mp}\right) \frac{\mathrm{d} k_{ \pm}}{k_{ \pm}^{1 / 2}} \\
& +\mathrm{i} \frac{\mathscr{S}}{4 \pi N^{2} \sin \theta_{0}} \frac{\exp \left(-\mathrm{i} \omega_{0} t\right)}{\left(x_{+}+x_{-}\right)^{1 / 2}\left(x_{\mp}-\mathrm{i} 0\right)^{1 / 2}} \\
& -\mathrm{i} \frac{\mathscr{S}}{4 \pi N^{2} \sin \theta_{0}} \frac{\exp \left(-\mathrm{i} \omega_{0} t\right)}{\left(x_{+} x_{-}+\mathrm{i} 0\right)^{1 / 2}} .
\end{aligned}
$$

It involves two one-dimensional Fourier integrals, with variables $x_{ \pm}$and $x_{\mp}$, respectively, of functions varying on a scale $1 / a$. Hence, by Lighthill's (1958, chap. 4) rule, at large values of the variables compared with $a$, the asymptotic behaviour of the integrals is attributable to the contributions from the singularity $k_{ \pm}=0$ of the two functions. Now, the far-field assumption $r / a \rightarrow \infty$ implies that either $\left|x_{ \pm}\right| / a=O(1)$ or $\left|x_{ \pm}\right| / r=O(1)$, while $\left|x_{\mp}\right| / r=O(1)$ everywhere. Accordingly the first, second and fifth terms of (4.29), normalized by $\mathscr{S} /\left[N^{2}(\text { ar })^{1 / 2}\right]$, are $O(1)$ for $\left|x_{ \pm}\right| / a=O(1)$, the second and fifth term, which may be written as

$$
-\mathrm{i} \frac{\mathscr{S}}{4 \pi N^{2} \sin \theta_{0}} \frac{\exp \left(-\mathrm{i} \omega_{0} t\right)}{\left(x_{+} x_{-}+\mathrm{i} 0\right)^{1 / 2}}\left[1-\left(1+\frac{x_{ \pm}}{x_{\mp}}\right)^{-1 / 2}\right],
$$

producing an $O(a / r)$ combination, and $O\left[(a / r)^{1 / 2}\right]$ for $\left|x_{ \pm}\right| / r=O(1)$, the first and second term, which may be written as

$$
\frac{1}{4 \pi^{3 / 2} N^{2} \sin \theta_{0}} \frac{\exp \left[-\mathrm{i}\left(\omega_{0} t+3 \pi / 4\right)\right]}{\left(x_{+}+x_{-}\right)^{1 / 2}} \int_{0}^{\infty}\left[q_{ \pm}\left(k_{ \pm}, \varphi, 0\right)-\mathscr{S}\right] \exp \left(\mathrm{i} k_{ \pm} x_{ \pm}\right) \frac{\mathrm{d} k_{ \pm}}{k_{ \pm}^{1 / 2}},
$$

producing an $O\left[(a / r)^{3 / 2}\right]$ combination; the third and fourth terms, on the other hand, which may be written as

$$
\frac{1}{4 \pi^{3 / 2} N^{2} \sin \theta_{0}} \frac{\exp \left[-\mathrm{i}\left(\omega_{0} t+\pi / 4\right)\right]}{\left(x_{+}+x_{-}\right)^{1 / 2}} \int_{0}^{\infty}\left[q_{ \pm}\left(k_{ \pm}, \varphi+\pi, 0\right)-\mathscr{S}\right] \exp \left(-\mathrm{i} k_{ \pm} x_{\mp}\right) \frac{\mathrm{d} k_{ \pm}}{k_{ \pm}^{1 / 2}},
$$

are everywhere $O\left[(a / r)^{1 / 2}\right]$ and produce everywhere an $O\left[(a / r)^{3 / 2}\right]$ combination.

Hence the third and fourth terms, always negligible compared with the dominant contribution, may safely be ignored, and two distinct expansions are obtained: one, uniform, valid wherever $r \gg a$,

$$
\begin{aligned}
\chi(\boldsymbol{x}, t) \sim & \frac{1}{4 \pi^{3 / 2} N^{2} \sin \theta_{0}} \frac{\exp \left[-\mathrm{i}\left(\omega_{0} t+3 \pi / 4\right)\right]}{\left(x_{+}+x_{-}\right)^{1 / 2}} \int_{0}^{\infty} q_{ \pm}\left(k_{ \pm}, \varphi, 0\right) \exp \left(\mathrm{i} k_{ \pm} x_{ \pm}\right) \frac{\mathrm{d} k_{ \pm}}{k_{ \pm}^{1 / 2}} \\
& +\mathrm{i} \frac{\mathscr{S}}{4 \pi N^{2} \sin \theta_{0}} \frac{\exp \left(-\mathrm{i} \omega_{0} t\right)}{\left(x_{+}+x_{-}\right)^{1 / 2}\left(x_{ \pm}+\mathrm{i} 0\right)^{1 / 2}} \\
& -\mathrm{i} \frac{\mathscr{S}}{4 \pi N^{2} \sin \theta_{0}} \frac{\exp \left(-\mathrm{i} \omega_{0} t\right)}{\left(x_{+} x_{-}+\mathrm{i} 0\right)^{1 / 2}}
\end{aligned}
$$


and the other, non-uniform, in which the space is divided into two zones, depending on whether the angular separation $\left|x_{ \pm}\right| / r$ from $\mathscr{C}_{0}$ is finite and $O(1)$, or infinitesimal and $O(a / r)$, respectively, as $r / a \rightarrow \infty$. In the outer zone, corresponding to $\left|z_{ \pm}\right| / a \rightarrow \infty$ with $\left|x_{ \pm}\right| /\left|z_{ \pm}\right|=O(1)$, the first two terms of (4.33) combine into a higher-order term, and the third term, given by (3.7), becomes dominant; in the inner zone, corresponding to $\left|z_{ \pm}\right| / a \rightarrow \infty$ with $\left|x_{ \pm}\right| / a=O(1)$, the last two terms combine into another higher-order term, and the first term, given by (4.16), becomes dominant.

A more intricate Fourier-space procedure has been substituted to the relatively straightforward real-space procedure of $\S 4.1$, generalizing the non-uniform expansions (3.7) and (4.16) in two ways: through the uniform expansion (4.33), and through the exact expression (4.21).

\subsection{Structure of the wave field}

Having established the mathematical conditions for the validity of the non-uniform expansion, we move on to analysing its physical implications. For this we replace the unphysical wave function by the associated pressure and velocity, deduced from it according to (2.11). In conical polar coordinates the velocity components are

$$
\begin{gathered}
u_{x_{ \pm}}=\left[\left(\frac{\partial}{\partial t}-v \nabla^{2}\right) \frac{\partial}{\partial t}+N^{2} \cos ^{2} \theta_{0}\right] \frac{\partial \chi}{\partial x_{ \pm}} \pm N^{2} \cos \theta_{0} \sin \theta_{0} \frac{\partial \chi}{\partial z_{ \pm}} \\
u_{\varphi}=\left[\left(\frac{\partial}{\partial t}-v \nabla^{2}\right) \frac{\partial}{\partial t}+N^{2}\right]\left(\frac{1}{r_{\mathrm{h}}} \frac{\partial \chi}{\partial \varphi}\right), \\
u_{z_{ \pm}}= \pm N^{2} \cos \theta_{0} \sin \theta_{0} \frac{\partial \chi}{\partial x_{ \pm}}+\left[\left(\frac{\partial}{\partial t}-v \nabla^{2}\right) \frac{\partial}{\partial t}+N^{2} \sin ^{2} \theta_{0}\right] \frac{\partial \chi}{\partial z_{ \pm}}
\end{gathered}
$$

where

$$
\nabla^{2}=\frac{\partial^{2}}{\partial x_{ \pm}^{2}}+\frac{\partial^{2}}{\partial z_{ \pm}^{2}}+\frac{1}{r_{\mathrm{h}}}\left(\cos \theta_{0} \frac{\partial}{\partial x_{ \pm}} \pm \sin \theta_{0} \frac{\partial}{\partial z_{ \pm}}\right)+\frac{1}{r_{\mathrm{h}}^{2}} \frac{\partial^{2}}{\partial \varphi^{2}},
$$

and where in this section the assumptions of steady monochromatic $(\partial / \partial t=$ $\left.-\mathrm{i} N \cos \theta_{0}\right)$ inviscid $(v=0)$ motion apply.

In the inner zone, where $\left|z_{ \pm}\right| \gg a$ and $\left|x_{ \pm}\right| / a=O(1)$, we have to leading order

$$
p \sim \mathrm{i} \rho_{0} N^{3} \chi \cos \theta_{0} \sin ^{2} \theta_{0}, \quad \boldsymbol{u} \sim \pm N^{2} \boldsymbol{e}_{z_{ \pm}} \frac{\partial \chi}{\partial x_{ \pm}} \cos \theta_{0} \sin \theta_{0},
$$

from which we derive

$$
\begin{aligned}
& p(\boldsymbol{x}, t) \sim \frac{\rho_{0} N}{2^{5 / 2} \pi^{3 / 2}}\left(\frac{\cos \theta_{0} \sin \theta_{0}}{\left|z_{ \pm}\right|}\right)^{1 / 2} \exp \left[-\mathrm{i}\left(\omega_{0} t+\frac{1}{4} \pi\right)\right] \\
& \times \int_{0}^{\infty} k_{ \pm}^{-1 / 2} q_{ \pm}\left(k_{ \pm}, \varphi, 0\right) \exp \left(\mathrm{i} k_{ \pm} x_{ \pm}\right) \mathrm{d} k_{ \pm} \\
& \boldsymbol{u}(\boldsymbol{x}, t) \sim \pm \frac{\boldsymbol{e}_{z_{ \pm}}}{2^{5 / 2} \pi^{3 / 2}}\left(\frac{\cot \theta_{0}}{\left|z_{ \pm}\right|}\right)^{1 / 2} \exp \left[-\mathrm{i}\left(\omega_{0} t+\frac{1}{4} \pi\right)\right] \\
& \times \int_{0}^{\infty} k_{ \pm}^{1 / 2} q_{ \pm}\left(k_{ \pm}, \varphi, 0\right) \exp \left(\mathrm{i} k_{ \pm} x_{ \pm}\right) \mathrm{d} k_{ \pm} .
\end{aligned}
$$

These formulae describe waves confined to a shell of constant thickness, of order $2 a$, around the double cone $\mathscr{C}_{0}$, a result first obtained for specific oscillating bodies by Hurley (1969) in two dimensions and Hendershott (1969) in three dimensions. 


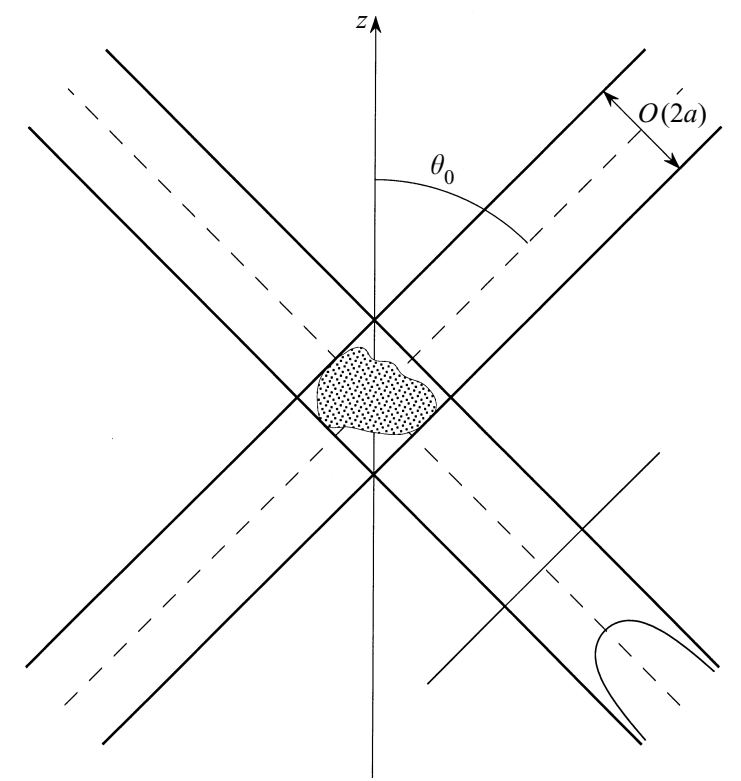

FiguRE 4. Wave beams from an extended source of radius $a$. The waves propagate inside a shell of constant thickness around the double cone $\mathscr{C}_{0}$, shown dashed. The wave energy flux for a pulsating sphere, taken from figure $5(\mathrm{~g})$, is represented on the lower beam.

A double wave beam is formed, represented in figure 4, inside which the phase propagates transversely, in the directions $O x_{+}$for the upper beam and $O x_{-}$for the lower beam. Energy, on the contrary, propagates longitudinally, in the direction $O z_{+}$ for the upper beam and opposite to the direction $O z_{-}$for the lower beam, as revealed by the consideration of the intensity, or average energy flux per unit area per unit time,

$$
\boldsymbol{I}=\frac{1}{2} \operatorname{Re}\left[p \boldsymbol{u}^{\star}\right]
$$

where ${ }^{\star}$ denotes a complex conjugate.

The average power at a given distance $\left|z_{+}\right|$results from the integration of the intensity with respect to the transverse coordinate $x_{ \pm}$and azimuthal angle $\varphi$, followed by the superposition of the contributions of the upper and lower beams. The transverse area element is

$$
\mathrm{d}^{2} S_{z_{ \pm}}=r_{\mathrm{h}} \mathrm{d} x_{ \pm} \mathrm{d} \varphi \sim\left|z_{ \pm}\right| \sin \theta_{0} \mathrm{~d} x_{ \pm} \mathrm{d} \varphi
$$

yielding the average power

$$
P \sim\left|z_{ \pm}\right| \sin \theta_{0} \int_{0}^{2 \pi} \mathrm{d} \varphi\left(\int_{-\infty}^{\infty} I_{z_{+}} \mathrm{d} x_{+}-\int_{-\infty}^{\infty} I_{z_{-}} \mathrm{d} x_{-}\right),
$$

conserved during propagation as implied by the $\left|z_{ \pm}\right|^{-1 / 2}$ amplitude decay of the pressure and velocity; accordingly, the asymptotic equivalence is replaced by an equality. From (4.37)-(4.38) we obtain

$$
P=\frac{\rho_{0} N \cos \theta_{0} \sin \theta_{0}}{32 \pi^{2}} \int_{0}^{2 \pi} \mathrm{d} \varphi_{k} \sum_{ \pm} \int_{0}^{\infty} \mathrm{d} k_{ \pm}\left|q_{ \pm}\left(k_{ \pm}, \varphi_{k}, 0\right)\right|^{2},
$$

a result first derived by Gorodtsov \& Teodorovich (1986) from the consideration of the rate of work exerted by the source, as discussed in Appendix C. 
As the wave beams are left, at the intermediate transverse distances $a \ll\left|x_{ \pm}\right| \ll\left|z_{ \pm}\right|$, the pressure and velocity become

$$
\begin{aligned}
& p(\boldsymbol{x}, t) \sim \frac{\rho_{0} N \mathscr{S}}{2^{5 / 2} \pi}\left(\frac{\cos \theta_{0} \sin \theta_{0}}{\left|z_{ \pm}\right|}\right)^{1 / 2} \frac{\exp \left(-\mathrm{i} \omega_{0} t\right)}{\left(x_{ \pm}+\mathrm{i} 0\right)^{1 / 2}}, \\
& \boldsymbol{u}(\boldsymbol{x}, t) \sim \pm \mathrm{i} \frac{\mathscr{S}}{2^{7 / 2} \pi}\left(\frac{\cot \theta_{0}}{\left|z_{ \pm}\right|}\right)^{1 / 2} \boldsymbol{e}_{z_{ \pm}} \frac{\exp \left(-\mathrm{i} \omega_{0} t\right)}{\left(x_{ \pm}+\mathrm{i} 0\right)^{3 / 2}}
\end{aligned}
$$

producing, to this approximation, no energy flux. Further away, in the outer zone where $\left|z_{ \pm}\right| \gg a$ and $\left|x_{ \pm}\right| /\left|z_{ \pm}\right|=O(1)$, they match with the pressure (3.9) and velocity (3.10) for a point source, respectively.

We investigate now the specific form of the waves in two particular cases.

\subsection{The pulsating sphere}

The most common source of internal waves in the laboratory is an oscillating body, generally a sphere or horizontal circular cylinder. A popular approximation consists in borrowing the representation of this body from irrotational homogeneous flow theory, so that, for example, a sphere of radius $a$, either pulsating at the radial velocity $U \exp \left(-\mathrm{i} \omega_{0} t\right)$ or vibrating at the translational velocity $\boldsymbol{U} \exp \left(-\mathrm{i} \omega_{0} t\right)$, is represented by the respective source terms

$$
q_{0}(\boldsymbol{x})=U \delta(r-a), \quad q_{0}(\boldsymbol{x})=\frac{3}{2} \boldsymbol{U} \cdot \frac{\boldsymbol{x}}{a} \delta(r-a) .
$$

This approximation was introduced by Gorodtsov \& Teodorovich (1982) for bodies in uniform translation, on the assumption of large internal Froude number, and later applied by Gorodtsov \& Teodorovich (1986), Ivanov (1989) and Makarov et al. (1990) to oscillating bodies; the same approximation is implicit when Lighthill $(1978$, p. 380) writes: 'if, for example, a cylindrical wire oscillating horizontally in the fluid has its cross-section compact [...], then it may be represented by a horizontal dipole of strength [...] equal to the force per unit length which it exerts on the fluid'.

For monopolar oscillations, i.e. pulsations, the approximation is certainly valid, as the monopole strength of a body involves only the changes of the volume of this body. For oscillations of higher multipolar order, however, the multipole strength involves both the motion of the body and the response of the fluid, a response modified by the stratification; the approximation consists in neglecting this modification. In Lighthill's wire example, the dipole strength is actually related to the time derivative of the fluid impulse, namely to the superposition of the pressure forces exerted at the wire and of the buoyancy forces generated within the fluid; implicitly, Lighthill's above statement neglects the second contribution. More generally, for dipolar oscillations, i.e. vibrations, the approximation of Gorodtsov \& Teodorovich $(1982,1986)$ consists in neglecting the influence of the stratification on the added mass of the vibrating body; this influence has since been shown to be effective, both theoretically (Lai \& Lee 1981) and experimentally (Ermanyuk 2000, 2002; Ermanyuk \& Gavrilov 2002a, b, 2003), and calculations neglecting it have been shown to be erroneous, for both inviscid (Hurley 1997; Hurley \& Keady 1997) and viscous (Chashechkin \& Kistovich 1997; Kistovich \& Chashechkin 1999a,b; Chashechkin et al. 2001) generation mechanisms.

For this reason we restrict our attention to the pulsating sphere, represented by the source $(4.45 a)$ of spectrum

$$
q_{0}(\boldsymbol{k})=4 \pi a^{2} U \frac{\sin (\kappa a)}{\kappa a} .
$$




\begin{tabular}{cccc}
\hline Source & Pressure amplitude & Velocity amplitude & Intensity \\
Spherical & $\frac{\rho_{0} N a U\left(\cos \theta_{0} \sin \theta_{0}\right)^{1 / 2}}{\left(\left|z_{ \pm}\right| / a\right)^{1 / 2}}$ & $\frac{U \cot ^{1 / 2} \theta_{0}}{2\left(\left|z_{ \pm}\right| / a\right)^{1 / 2}}$ & $\frac{\rho_{0} N a^{2} U^{2} \cos \theta_{0}}{4\left|z_{ \pm}\right|}$ \\
Gaussian & $\frac{\rho_{0} N \mathscr{S}\left(\cos \theta_{0} \sin \theta_{0}\right)^{1 / 2}}{2^{11 / 4} \pi^{1 / 2} \Gamma\left(\frac{3}{4}\right) a^{1 / 2}\left|z_{ \pm}\right|^{1 / 2}}$ & $\frac{\mathscr{S} \cot ^{1 / 2} \theta_{0}}{2^{9 / 4} \pi^{1 / 2} \Gamma\left(\frac{1}{4}\right) a^{3 / 2}\left|z_{ \pm}\right|^{1 / 2}}$ & $\frac{\rho_{0} N \mathscr{S}^{2} \cos \theta_{0}}{2^{13 / 2} \pi^{2} a^{2}\left|z_{ \pm}\right|}$ \\
Unsteady & $\frac{\rho_{0} N \mathscr{S}\left(\cos \theta_{0} \sin \theta_{0}\right)^{1 / 2}}{(2 \pi)^{3 / 2} \alpha^{1 / 2}\left|z_{ \pm}\right|}$ & $\frac{\mathscr{S} \cot ^{1 / 2} \theta_{0}}{3(2 \pi)^{3 / 2} \alpha^{3 / 2} z_{ \pm}^{2}}$ & $\frac{\rho_{0} N \mathscr{S}^{2} \cos \theta_{0}}{48 \pi^{3} \alpha^{2}\left|z_{ \pm}\right|^{3}}$ \\
Viscous & $\frac{\rho_{0} N \mathscr{S}\left(\cos \theta_{0} \sin \theta_{0}\right)^{1 / 2}}{2^{3 / 2} 3 \pi^{1 / 2} \Gamma\left(\frac{5}{6}\right) \beta^{1 / 6}\left|z_{ \pm}\right|^{2 / 3}}$ & $\frac{\mathscr{S} \cot ^{1 / 2} \theta_{0}}{2^{5 / 2} 3 \pi \beta^{1 / 2}\left|z_{ \pm}\right|}$ & $\frac{\rho_{0} N \mathscr{S}^{2} \cos \theta_{0}}{288 \pi^{3 / 2} \Gamma\left(\frac{5}{6}\right) \beta^{2 / 3}\left|z_{ \pm}\right|^{5 / 3}}$
\end{tabular}

TABLE 1. Waves at the centreline $x_{ \pm}=0$ of the beams, for the pulsating sphere of $\S 4.4$, the Gaussian monopole of $\S 4.5$, the unsteady monopole of $\S 5.3$, and the viscous monopole of $\S 6.2$.

The pressure and velocity are obtained from (4.37)-(4.38) and table 5 as

$$
\begin{aligned}
& p(\boldsymbol{x}, t) \sim \frac{\rho_{0} N a U}{2^{1 / 2}}\left(\frac{\cos \theta_{0} \sin \theta_{0}}{\left|z_{ \pm}\right| / a}\right)^{1 / 2} \exp \left(-\mathrm{i} \omega_{0} t\right) \\
& \times\left[\left(\frac{x_{ \pm}+a+\mathrm{i} 0}{a}\right)^{1 / 2}-\left(\frac{x_{ \pm}-a+\mathrm{i} 0}{a}\right)^{1 / 2}\right], \\
& \boldsymbol{u}(\boldsymbol{x}, t) \sim \pm \mathrm{i} \frac{U}{2^{3 / 2}}\left(\frac{\cot \theta_{0}}{\left|z_{ \pm}\right| / a}\right)^{1 / 2} \boldsymbol{e}_{z_{ \pm}} \exp \left(-\mathrm{i} \omega_{0} t\right) \\
& \times\left[\left(\frac{a}{x_{ \pm}-a+\mathrm{i} 0}\right)^{1 / 2}-\left(\frac{a}{x_{ \pm}+a+\mathrm{i} 0}\right)^{1 / 2}\right] .
\end{aligned}
$$

The identity with the results of Hendershott (1969), Appleby \& Crighton (1987) and Voisin (1991), obtained by direct solution of the wave equation with boundary condition of fixed radial velocity at the surface of the sphere, follows from the identity

$$
\left(\frac{x+1}{2}\right)^{1 / 2}-\left(\frac{x-1}{2}\right)^{1 / 2}=\left[x-\left(x^{2}-1\right)^{1 / 2}\right]^{1 / 2}=\exp \left(-\frac{\operatorname{arccosh} x}{2}\right),
$$

and validates the model $(4.45 a)$ a posteriori.

The pressure and velocity are represented in figure $5(a-f)$, together with the respective asymptotic expansions (4.43)-(4.44) where $\mathscr{S}=4 \pi a^{2} U$; they are normalized by the centreline values given in table 1 . Though wavelike, in the sense that the successive waveforms exhibit the propagation of information from one edge $x_{ \pm}=-a$ of each beam to the opposite edge $x_{ \pm}=a$ as time progresses, the profiles also exhibit clearly that the phase and amplitude vary on the same spatial scale, the radius $a$ of the sphere, implying that the waves are not quasi-plane.

Inside the beams, that is between the two characteristic double cones $\left|x_{ \pm}\right|=a$ tangent to the sphere above and below, the pressure and velocity oscillate in phase, with total phase variation $\pi / 2$ - a quarter of an oscillation - across each beam. Because of this, Appleby \& Crighton (1987) introduced an effective wavelength $8 a$, obtained by multiplying the distance $2 a$ over which the phase varies by the ratio of the variation $2 \pi$ over one wavelength to the actual variation $\pi / 2$; the effective wavelength 



Figure 5. Waves from a pulsating sphere of radius $a$, as a function of $x_{ \pm} / a$. The phase and amplitude profiles, and the successive waveforms at the successive values $0, \pi / 4, \pi / 2,3 \pi / 4$ and $\pi$ of $\omega_{0} t+3 \pi / 4$, are represented in $(a),(c)$ and $(e)$, respectively, for the pressure $p$, and $(b)$, $(d)$ and $(f)$, respectively, for the outgoing velocity $\pm u_{z_{+}} ;(g)$ is the profile of outgoing intensity $\pm I_{z_{+}}$. The phases are normalized by $\pi$ and their origin is taken at $-\left(\omega_{0} t+\pi / 4\right)$; the amplitudes and intensity are normalized by their centreline values, given in table 1 . In $(a)-(d)$ and $(g)$ the dashed curves represent the asymptotic expansions (4.43)-(4.44), valid far from the centreline; in $(e)-(f)$ they represent the envelopes of the waveforms.

replaces the usual one $\lambda=2 \mathrm{i} \pi p /\left(\partial p / \partial x_{ \pm}\right)=4 \pi\left(a^{2}-x_{+}^{2}\right)^{1 / 2}$, meaningless for a nonquasi-plane wave. Similarly, the usual phase velocity $c_{\phi}=\omega_{0} \lambda /(2 \pi)=2 \omega_{0}\left(a^{2}-x_{+}^{2}\right)^{1 / 2}$ is meaningless; in another context Thomas \& Stevenson (1972) have introduced the velocities of propagation of the crests and troughs on one hand, and of the nodes on the other, and verified that these are indeed different at any given position $x_{ \pm}$. 
At the edges of the beams, that is on the two double cones $\left|x_{ \pm}\right|=a$, the phase of the velocity jumps abruptly by $\pi / 2$, and its amplitude diverges but remains integrable. Outside the beams the pressure and velocity oscillate in quadrature and have constant phases. Accordingly, the energy flux, given by

$$
\boldsymbol{I}(\boldsymbol{x}) \sim \pm \frac{\rho_{0} N a^{2} U^{2} \cos \theta_{0}}{4\left|z_{ \pm}\right|} \boldsymbol{e}_{z_{ \pm}} \frac{H\left(a^{2}-x_{ \pm}^{2}\right)}{\left(1-x_{ \pm}^{2} / a^{2}\right)^{1 / 2}},
$$

and represented in figure $5(\mathrm{~g})$, is confined to the beams: the power output of a pulsating sphere of diameter $2 a$, given by

$$
P=\pi^{2} \rho_{0} N a^{3} U^{2} \cos \theta_{0} \sin \theta_{0},
$$

is distributed across a conical shell of exactly the same width $2 a$, as shown in figure 4 . The singularity of the velocity is a feature of any source of monochromatic internal waves with sharp boundaries, e.g. any oscillating body.

\subsection{The Gaussian monopole}

An alternative way of giving the point source of $\S 3$ a spatial extent, also used for sources in uniform translation (see e.g. Gorodtsov \& Teodorovich 1980), is to allow this source to have a Gaussian distribution in space with standard deviation $a$. The source term,

$$
q_{0}(\boldsymbol{x})=\frac{\mathscr{S}}{(2 \pi)^{3 / 2} a^{3}} \exp \left(-\frac{r^{2}}{2 a^{2}}\right),
$$

has a spectrum which is itself a Gaussian function,

$$
q_{0}(\boldsymbol{k})=\mathscr{S} \exp \left(-\frac{\kappa^{2} a^{2}}{2}\right) .
$$

The pressure and velocity are obtained from (4.37)-(4.38) and table 5 as

$$
\begin{aligned}
& p(\boldsymbol{x}, t) \sim \frac{\rho_{0} N \mathscr{S}}{2^{5 / 2} \pi} \frac{\left(\cos \theta_{0} \sin \theta_{0}\right)^{1 / 2}}{a^{1 / 2}\left|z_{ \pm}\right|^{1 / 2}} \exp \left[-\mathrm{i}\left(\omega_{0} t+\frac{1}{4} \pi\right)\right] \exp \left(-\frac{x_{ \pm}^{2}}{4 a^{2}}\right) D_{-1 / 2}\left(-\mathrm{i} \frac{x_{ \pm}}{a}\right), \\
& \boldsymbol{u}(\boldsymbol{x}, t) \sim \pm \frac{\mathscr{S}}{2^{7 / 2} \pi} \frac{\cot ^{1 / 2} \theta_{0}}{a^{3 / 2}\left|z_{ \pm}\right|^{1 / 2}} \boldsymbol{e}_{z_{ \pm}} \exp \left[-\mathrm{i}\left(\omega_{0} t+\frac{1}{4} \pi\right)\right] \exp \left(-\frac{x_{ \pm}^{2}}{4 a^{2}}\right) D_{-3 / 2}\left(-\mathrm{i} \frac{x_{ \pm}}{a}\right),
\end{aligned}
$$

where the parabolic cylinder functions $D_{\mu}(x)$ are related to the modified Bessel functions $I_{\mu}(x)$ by

$$
\begin{gathered}
D_{-1 / 2}(-\mathrm{i} x)=\frac{(\pi x)^{1 / 2}}{2}\left(I_{-1 / 4}+\mathrm{i} I_{1 / 4}\right)\left(\frac{x^{2}}{4}\right), \\
D_{-3 / 2}(-\mathrm{i} x)=\frac{\pi^{1 / 2} x^{3 / 2}}{2}\left[I_{-3 / 4}-I_{1 / 4}+\mathrm{i}\left(I_{-1 / 4}-I_{3 / 4}\right)\right]\left(\frac{x^{2}}{4}\right) .
\end{gathered}
$$

The energy flux is also a Gaussian function

$$
\boldsymbol{I}(\boldsymbol{x}) \sim \pm \frac{\rho_{0} N \mathscr{S}^{2} \cos \theta_{0}}{2^{13 / 2} \pi^{2} a^{2}\left|z_{ \pm}\right|} \boldsymbol{e}_{z_{ \pm}} \exp \left(-\frac{x_{ \pm}^{2}}{2 a^{2}}\right),
$$



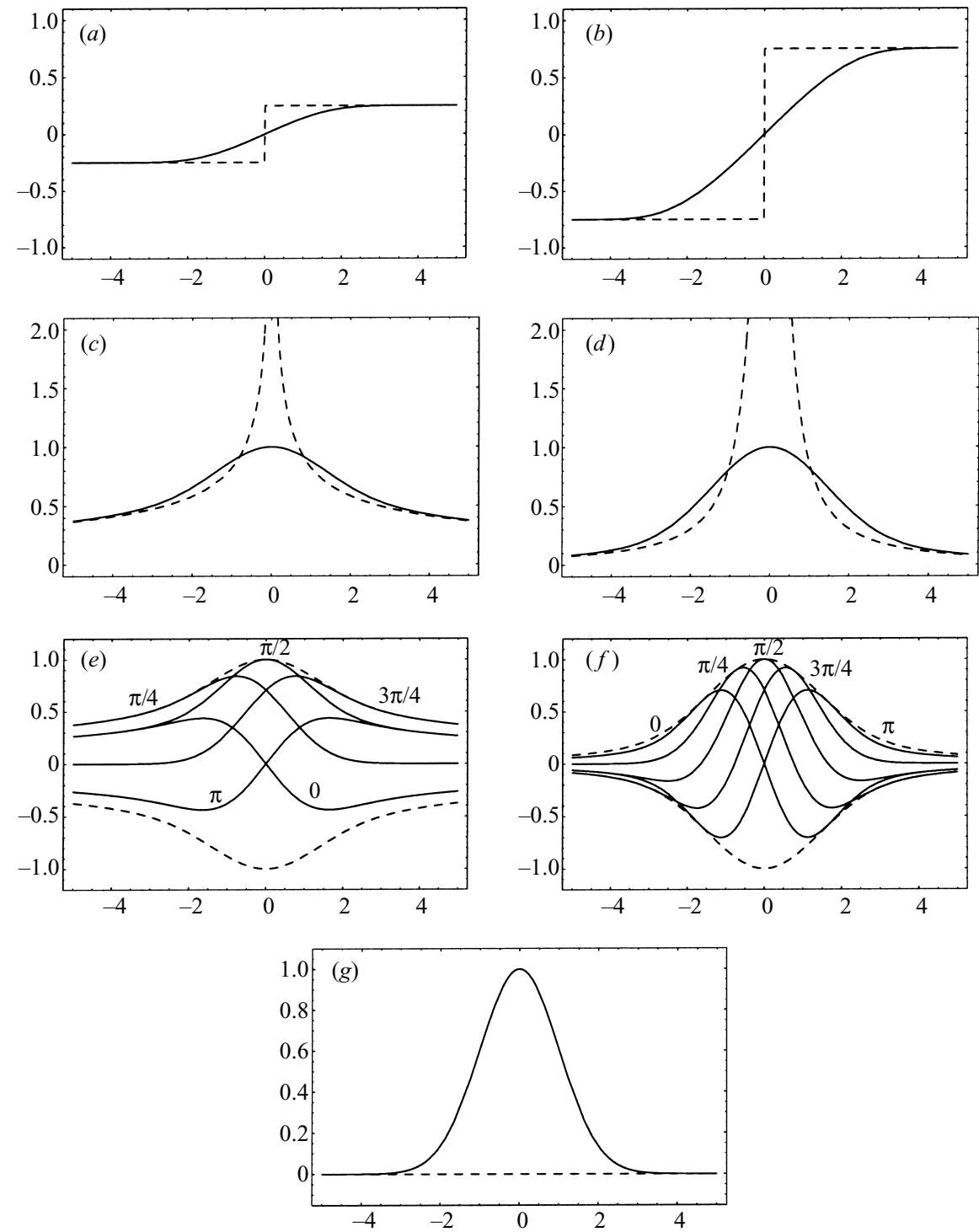

FIGURE 6. Waves from a Gaussian monopole of standard deviation $a$, as a function of $x_{ \pm} / a$. The mode of representation is the same as in figure 5.

distributing across the wave beams, with exactly the same standard deviation $a$ as the source, the power output

$$
P=\frac{\rho_{0} N \mathscr{S}^{2} \cos \theta_{0} \sin \theta_{0}}{16 \pi^{1 / 2} a} .
$$

The wave profiles are represented in figure 6 . They exhibit the same basic structure as for the pulsating sphere, without the unpleasant singularities: non-quasi-plane waves are radiated within a conical shell; along the centreline of this shell the pressure and velocity oscillate in phase and have maximum amplitudes; away from the centreline they become gradually shifted in phase; asymptotically phase quadrature and algebraic amplitude decay are reached. Unfortunately, the very absence 
of the singularities indicates that the Gaussian model, however revealing of the propagation of the waves it may be, must be abandoned for their serious study.

\section{Waves from an unsteady source}

We consider a monochromatic point monopole $q(\boldsymbol{x}, t)=\mathscr{S} \delta(\boldsymbol{x}) H(t) \exp \left(-\mathrm{i} \omega_{0} t\right)$ of strength $\mathscr{S} \exp \left(-\mathrm{i} \omega_{0} t\right)$ and frequency $0<\omega_{0}<N$, switched on impulsively at time $t=0$ in an inviscid fluid. The wave equation for this source,

$$
\left(\frac{\partial^{2}}{\partial t^{2}} \nabla^{2}+N^{2} \nabla_{\mathrm{h}}^{2}\right) \chi(\boldsymbol{x}, t)=\mathscr{S} \delta(\boldsymbol{x}) H(t) \exp \left(-\mathrm{i} \omega_{0} t\right),
$$

will be solved first in real space and time then in Fourier space and time.

\subsection{The real-space real-time way}

In real space and time, the solution to (5.1) follows from the convolution of the source term with the impulsive Green's function, yielding

$$
\chi(\boldsymbol{x}, t)=\mathscr{S} \int_{0}^{t} \exp \left(-\mathrm{i} \omega_{0} t^{\prime}\right) G\left(\boldsymbol{x}, t-t^{\prime}\right) \mathrm{d} t^{\prime},
$$

with $t$ the observation time and $t^{\prime}$ the emission time; here, as everywhere in this section, a factor $H(t)$ is implicit in the expression of the waves. We write

$$
\chi(\boldsymbol{x}, t)=\mathscr{S}\left(\int_{-\infty}^{t}-\int_{-\infty}^{0}\right) \exp \left(-\mathrm{i} \omega_{0} t^{\prime}\right) G\left(\boldsymbol{x}, t-t^{\prime}\right) \mathrm{d} t^{\prime},
$$

so as to separate the steady waves which would be observed if the source had been operating since $t^{\prime}=-\infty$, from the transient correction created by the fact that it has started to operate later at $t^{\prime}=0$. The steady waves have been calculated as (3.2) in terms of the monochromatic Green's function (2.17); the transient correction is evaluated for large times $N t \gg 1$ based on the asymptotic impulsive Green's function (2.18). We obtain, by table 5 ,

$$
\begin{aligned}
\chi(\boldsymbol{x}, t) \sim-\mathrm{i} & \frac{\mathscr{S}}{4 \pi N r \sin \theta_{0}} \frac{\exp \left(-\mathrm{i} \omega_{0} t\right)}{\left(\omega_{0}^{2}-N^{2} z^{2} / r^{2}+\mathrm{i} 0\right)^{1 / 2}} \\
& +\mathrm{i} \frac{\mathscr{S}}{2^{5 / 2} \pi^{3 / 2} N r_{\mathrm{h}}} \frac{\exp \left(-\mathrm{i} \omega_{0} t\right)}{(N|z| / r)^{1 / 2}} \frac{\Gamma\left[\frac{1}{2},-\mathrm{i} t\left(\omega_{0}-N|z| / r+\mathrm{i} 0\right)\right]}{\left(\omega_{0}-N|z| / r+\mathrm{i} 0\right)^{1 / 2}} \\
& +\frac{\mathscr{S}}{2^{5 / 2} \pi^{3 / 2} N r_{\mathrm{h}}} \frac{\exp \left(-\mathrm{i} \omega_{0} t\right)}{(N|z| / r)^{1 / 2}} \frac{\Gamma\left[\frac{1}{2},-\mathrm{i} t\left(\omega_{0}+N|z| / r+\mathrm{i} 0\right)\right]}{\left(\omega_{0}+N|z| / r+\mathrm{i} 0\right)^{1 / 2}} \\
& -\frac{\mathscr{S}}{2^{5 / 2} \pi^{3 / 2} N r_{\mathrm{h}}} \frac{\exp \left(-\mathrm{i} \omega_{0} t\right)}{N^{1 / 2}} \frac{\Gamma\left[\frac{1}{2},-\mathrm{i} t\left(\omega_{0}-N+\mathrm{i} 0\right)\right]}{\left(\omega_{0}-N+\mathrm{i} 0\right)^{1 / 2}} \\
& -\mathrm{i} \frac{\mathscr{S}}{2^{5 / 2} \pi^{3 / 2} N r_{\mathrm{h}}} \frac{\exp \left(-\mathrm{i} \omega_{0} t\right)}{N^{1 / 2}} \frac{\Gamma\left[\frac{1}{2},-\mathrm{i} t\left(\omega_{0}+N+\mathrm{i} 0\right)\right]}{\left(\omega_{0}+N+\mathrm{i} 0\right)^{1 / 2}},
\end{aligned}
$$

a uniform expansion of the wave function where the first term, representing steady waves at the forcing frequency $\omega_{0}$, is supplemented by four other terms, involving complementary incomplete gamma functions and representing transient gravity waves of frequencies $N|z| / r$ and $-N|z| / r$, and transient buoyancy oscillations of frequencies $N$ and $-N$, respectively, modified by their excitation by an oscillatory source.

The first two terms diverge individually on the double cone $\mathscr{C}_{0}$, where their frequencies become equal allowing them to interfere with each other so as to produce 
a finite result. Again, the space must be divided into two zones, depending on whether the angular separation from $\mathscr{C}_{0}$ is finite and $O(1)$, or infinitesimal and $O[1 /(N t)]$, respectively, as $N t \rightarrow \infty$. We express the separation in conical polar coordinates $\left(x_{ \pm}, \varphi, z_{ \pm}\right)$, with $\pm=\operatorname{sign} z$.

In the outer zone, corresponding to $N t \rightarrow \infty$ with $\left|x_{ \pm}\right| /\left|z_{ \pm}\right|=O(1)$, we have, from the asymptotic expansion of the complementary incomplete gamma functions,

$$
\begin{aligned}
\chi(\boldsymbol{x}, t) \sim-\mathrm{i} \frac{\mathscr{S}}{4 \pi N^{2} \sin \theta_{0}} \frac{\exp \left(-\mathrm{i} \omega_{0} t\right)}{\left(x_{+} x_{-}+\mathrm{i} 0\right)^{1 / 2}}+\frac{\mathscr{S}}{2^{5 / 2} \pi^{3 / 2} N r_{\mathrm{h}}} \frac{1}{(N t|z| / r)^{1 / 2}} \\
\times\left\{\frac{\exp [-\mathrm{i}(N t|z| / r+\pi / 4)]}{N|z| / r-\omega_{0}}+\frac{\exp [\mathrm{i}(N t|z| / r+\pi / 4)]}{N|z| / r+\omega_{0}}\right\} \\
+\frac{\mathscr{S}}{2^{5 / 2} \pi^{3 / 2} N r_{\mathrm{h}}} \frac{1}{(N t)^{1 / 2}}\left\{\frac{\exp [-\mathrm{i}(N t-\pi / 4)]}{N-\omega_{0}}+\frac{\exp [\mathrm{i}(N t-\pi / 4)]}{N+\omega_{0}}\right\},
\end{aligned}
$$

a superposition of the steady waves (3.7), of frequency $\omega_{0}$, and of transient waves of frequencies $N|z| / r$ and $-N|z| / r$, and transient oscillations of frequencies $N$ and $-N$, respectively, similar to those in (2.18) and decreasing as $t^{-1 / 2}$. It must not be concluded, however, that in this zone the transient waves are negligible compared with the steady waves, as will be seen in $\S 5.3$.

In the inner zone, corresponding to $N t \rightarrow \infty$ with $N t\left|x_{ \pm}\right| /\left|z_{ \pm}\right|=O(1)$, the transient waves of frequency $-N|z| / r$ and transient oscillations of frequencies $N$ and $-N$ are unchanged, while the transient waves of frequency $N|z| / r \sim \omega_{0}-N x_{ \pm} /\left|z_{ \pm}\right| \sin \theta_{0}$ combine with the steady waves of nearby frequency $\omega_{0}$ to produce, through beating, modulated quasi-steady waves of main frequency $\omega_{0}$ and modulation frequency $N\left|x_{ \pm}\right| /\left|z_{ \pm}\right| \sin \theta_{0}$. The modulation is accounted for by an incomplete gamma function, in the form

$$
\begin{aligned}
\chi(x, t) \sim-\mathrm{i} & \frac{\mathscr{S}}{2^{5 / 2} \pi^{3 / 2} N^{2} \cos ^{1 / 2} \theta_{0} \sin ^{3 / 2} \theta_{0}} \frac{\exp \left(-\mathrm{i} \omega_{0} t\right)}{x_{ \pm}^{1 / 2}\left|z_{ \pm}\right|^{1 / 2}} \gamma\left(\frac{1}{2},-\mathrm{i} \frac{x_{ \pm}}{\alpha\left|z_{ \pm}\right|}\right) \\
& +\frac{\mathscr{S}}{2^{5 / 2} \pi^{3 / 2} N r_{\mathrm{h}}} \frac{1}{(N t|z| / r)^{1 / 2}} \frac{\exp [\mathrm{i}(N t|z| / r+\pi / 4)]}{N|z| / r+\omega_{0}} \\
& +\frac{\mathscr{S}}{2^{5 / 2} \pi^{3 / 2} N r_{\mathrm{h}}} \frac{1}{(N t)^{1 / 2}}\left\{\frac{\exp [-\mathrm{i}(N t-\pi / 4)]}{N-\omega_{0}}+\frac{\exp [\mathrm{i}(N t-\pi / 4)]}{N+\omega_{0}}\right\},
\end{aligned}
$$

where

$$
\alpha=\frac{1}{N t \sin \theta_{0}} .
$$

After appropriate changes of notation and variables this result is equivalent to that (13) of Makhortykh \& Rybak (1990), obtained by temporal Fourier transformation. Based on it Makhortykh \& Rybak investigated the waves on $\mathscr{C}_{0}$, i.e. for $x_{ \pm}=0$, as did Simakov (1993) without resorting to the intermediate step (5.6). In $\S 5.3$, we will investigate the structure of the wave field throughout the wave zone.

\subsection{The Fourier-space Fourier-time way}

Before doing so we look for an additional derivation of the wave function in Fourier space and time, paralleling the derivation in $\S 4.2$ for an extended source. The solution to (5.1) is written by table 5 as the inverse transform

$$
\chi(\boldsymbol{x}, t)=\mathrm{i} \frac{\mathscr{S}}{16 \pi^{4}} \int \frac{G(\boldsymbol{k}, \omega)}{\omega-\omega_{0}+\mathrm{i} 0} \exp [\mathrm{i}(\boldsymbol{k} \cdot \boldsymbol{x}-\omega t)] \mathrm{d}^{3} k \mathrm{~d} \omega,
$$


that is, by (2.19),

$$
\chi(\boldsymbol{x}, t)=\mathrm{i} \frac{\mathscr{S}}{16 \pi^{4}} \int \frac{\exp [\mathrm{i}(\boldsymbol{k} \cdot \boldsymbol{x}-\omega t)]}{\left(\omega-\omega_{0}+\mathrm{i} 0\right)\left[(\omega+\mathrm{i} 0)^{2} \kappa^{2}-N^{2} \kappa_{\mathrm{h}}^{2}\right]} \mathrm{d}^{3} k \mathrm{~d} \omega .
$$

The expansion of this transform for large times $N t \gg 1$ is obtained by adding up the asymptotic contributions of the singular frequencies of the integrand (Lighthill 1958, chap. 4). We are interested in the contribution $\chi_{0}(x, t)$ from the vicinity $\left|\omega-\omega_{0}\right| / N=O[1 /(N t)]$ of the forcing frequency $\omega_{0}$.

In cylindrical coordinates $\left(\kappa_{\mathrm{h}}, \varphi_{k}, m\right)$, this contribution is given by

$$
\begin{aligned}
\chi_{0}(\boldsymbol{x}, t) \sim \mathrm{i} \frac{\mathscr{S}}{16 \pi^{4} N^{2}} & \int_{0}^{\infty} \kappa_{\mathrm{h}} \mathrm{d} \kappa_{\mathrm{h}} \int_{0}^{2 \pi} \mathrm{d} \varphi_{k} \int_{-\infty}^{\infty} \mathrm{d} m \int_{-\infty}^{\infty} \frac{\mathrm{d} \omega}{\omega-\omega_{0}+\mathrm{i} 0} \\
& \times \frac{\exp \left\{\mathrm{i}\left[\kappa_{\mathrm{h}} r_{\mathrm{h}} \cos \left(\varphi_{k}-\varphi\right)+m z-\omega t\right]\right\}}{m^{2} \cos ^{2} \theta_{0}-\kappa_{\mathrm{h}}^{2} \sin ^{2} \theta_{0}+2\left(\omega-\omega_{0}\right) \kappa^{2} / N \cos \theta_{0}+\mathrm{i} 0} .
\end{aligned}
$$

In the complex $m$-plane the path of integration is the same as in figure 3 , except for an $O[1 /(N t)]$ displacement of the poles of the integrand, to the new positions $\kappa_{\mathrm{h}}\left[\tan \theta_{0}-\left(\omega-\omega_{0}\right) /\left(N \cos ^{2} \theta_{0} \sin \theta_{0}\right)\right]-\mathrm{i} 0$ and $-\kappa_{\mathrm{h}}\left[\tan \theta_{0}-\left(\omega-\omega_{0}\right) /\left(N \cos ^{2} \theta_{0} \sin \theta_{0}\right)\right]$ $+\mathrm{i}$. Deformation of this path selects the pole

$$
m=-\kappa_{\mathrm{h}}\left(\tan \theta_{0}-\frac{\omega-\omega_{0}}{N \cos ^{2} \theta_{0} \sin \theta_{0}}\right) \operatorname{sign} z,
$$

and evaluation of the residue at this pole followed by integration with respect to $\omega$ yields, by table 4 , in conical polar coordinates $\left(k_{ \pm}, \varphi_{k}, m_{ \pm}\right)$with $\pm=\operatorname{sign} z$,

$$
\chi_{0}(\boldsymbol{x}, t) \sim-\mathrm{i} \frac{\mathscr{S}}{8 \pi^{2} N^{2} \sin \theta_{0}} \exp \left(-\mathrm{i} \omega_{0} t\right) \int_{0}^{\cos \theta_{0} /(\alpha|z|)} \mathrm{d} k_{ \pm} \int_{0}^{2 \pi} \mathrm{d} \varphi_{k} \exp \left[\mathrm{i} k_{ \pm} \Phi\left(\varphi_{k} \mid \boldsymbol{x}\right)\right],
$$

where $\Phi\left(\varphi_{k} \mid \boldsymbol{x}\right)$ is defined by (4.22). The physical interpretation of this result is straightforward: since a plane internal wave of wavenumber $k_{ \pm}$travels at the group velocity $\left(N / k_{ \pm}\right) \sin \theta_{0}$ along rays (Lighthill 1978, §4.4), at a given vertical level $|z|$ reached after travelling the distance $|z| / \cos \theta_{0}$ along the double cone $\mathscr{C}_{0}$, at a given time $t$ after the start-up, only the waves of wavenumber smaller than $(N t /|z|) \cos \theta_{0} \sin \theta_{0}=\cos \theta_{0} /(\alpha|z|)$ have been received.

Mathematically, the wave function (5.12) is of the same form (4.21) as for an extended source, with the cutoff factor $\mathscr{S} H\left[\cos \theta_{0} /(\alpha|z|)-k_{ \pm}\right]$playing the role of a spectrum. It is evaluated in the same way, to one difference: there is no 'global' contribution, and $d$ is replaced by $\alpha|z| / \cos \theta_{0}$ in the 'local' contribution. Two distinct expansions are obtained: one, uniform, valid whenever $N t \gg 1$,

$$
\begin{aligned}
\chi_{0}(\boldsymbol{x}, t) \sim-\mathrm{i} & \frac{\mathscr{S}}{4 \pi^{3 / 2} N^{2} \sin \theta_{0}} \frac{\exp \left(-\mathrm{i} \omega_{0} t\right)}{\left(x_{+}+x_{-}\right)^{1 / 2} x_{ \pm}^{1 / 2}} \gamma\left(\frac{1}{2},-\mathrm{i} \frac{x_{ \pm} \cos \theta_{0}}{\alpha|z|}\right) \\
& +\mathrm{i} \frac{\mathscr{S}}{4 \pi N^{2} \sin \theta_{0}} \frac{\exp \left(-\mathrm{i} \omega_{0} t\right)}{\left(x_{+}+x_{-}\right)^{1 / 2}\left(x_{ \pm}+\mathrm{i} 0\right)^{1 / 2}} \\
& -\mathrm{i} \frac{\mathscr{S}}{4 \pi N^{2} \sin \theta_{0}} \frac{\exp \left(-\mathrm{i} \omega_{0} t\right)}{\left(x_{+} x_{-}+\mathrm{i} 0\right)^{1 / 2}},
\end{aligned}
$$

where $|z| / \cos \theta_{0}=\left(x_{\mp}-x_{ \pm}\right) / \sin \left(2 \theta_{0}\right)$, and the other, non-uniform, in which the space is divided into two zones, depending on whether the angular separation $\left|x_{ \pm}\right| / r$ from $\mathscr{C}_{0}$ is finite and $O(1)$, or infinitesimal and $O[1 /(N t)]$, respectively, as $N t \rightarrow \infty$. In the outer zone, corresponding to $\alpha \rightarrow 0$ with $\left|x_{ \pm}\right| /\left|z_{ \pm}\right|=O(1)$, the first two terms of 
(5.13) combine into a higher-order term, and the third term, given by (3.7), becomes dominant; in the inner zone, corresponding to $\alpha \rightarrow 0$ with $\left|x_{ \pm}\right| /\left(\alpha\left|z_{ \pm}\right|\right)=O(1)$, the last two terms combine into another higher-order term, and the first term, given by that in (5.6), becomes dominant.

In this way, the results of $\S 5.1$ are recovered and complemented by the two alternative expansions (5.12) and (5.13).

\subsection{Structure of the wave field}

We investigate now the structure of the wave field. In the inner zone, where $N t \gg 1$ and $\left|x_{ \pm}\right| /\left(\alpha\left|z_{ \pm}\right|\right)=O(1)$, the first, quasi-steady, term of the wave function (5.6) gives, to leading order, by (4.36), the pressure and velocity

$$
\begin{aligned}
& p(\boldsymbol{x}, t) \sim \frac{\rho_{0} N \mathscr{S}}{2^{5 / 2} \pi^{3 / 2}} \frac{\left(\cos \theta_{0} \sin \theta_{0}\right)^{1 / 2}}{x_{ \pm}^{1 / 2}\left|z_{ \pm}\right|^{1 / 2}} \exp \left(-\mathrm{i} \omega_{0} t\right) \gamma\left(\frac{1}{2},-\mathrm{i} \frac{x_{ \pm}}{\alpha\left|z_{ \pm}\right|}\right), \\
& \boldsymbol{u}(\boldsymbol{x}, t) \sim \pm \mathrm{i} \frac{\mathscr{S}}{2^{5 / 2} \pi^{3 / 2}} \frac{\cot ^{1 / 2} \theta_{0}}{x_{ \pm}^{3 / 2}\left|z_{ \pm}\right|^{1 / 2}} \boldsymbol{e}_{z_{ \pm}} \exp \left(-\mathrm{i} \omega_{0} t\right) \gamma\left(\frac{3}{2},-\mathrm{i} \frac{x_{ \pm}}{\alpha\left|z_{ \pm}\right|}\right),
\end{aligned}
$$

while the other, transient, terms give pressures and velocities which are $O[1 /(N t)]$ compared with (5.14)-(5.15), and thus negligible. Accordingly, the effect of the impulsive start-up is a modulation of the steady waves of frequency $\omega_{0}$ at the much lower frequency $N\left|x_{ \pm}\right| /\left|z_{ \pm}\right| \sin \theta_{0}$, a modulation accounted for by incomplete gamma functions related to the Fresnel integrals $C(x)$ and $S(x)$ by

$$
\begin{gathered}
\gamma\left(\frac{1}{2},-\mathrm{i} x\right)=(2 \pi)^{1 / 2} \exp \left(-\mathrm{i} \frac{1}{4} \pi\right)(C+\mathrm{i} S)\left[\left(\frac{2 x}{\pi}\right)^{1 / 2}\right] \\
\gamma\left(\frac{3}{2},-\mathrm{i} x\right)=\left(\frac{\pi}{2}\right)^{1 / 2} \exp \left(-\mathrm{i} \frac{1}{4} \pi\right)\left\{(C+\mathrm{i} S)\left[\left(\frac{2 x}{\pi}\right)^{1 / 2}\right]-\left(\frac{2 x}{\pi}\right)^{1 / 2} \exp (\mathrm{i} x)\right\} .
\end{gathered}
$$

Owing to the spatial variations of the modulation frequency, waves beams are formed, shown in figure 7 , made of a conical shell around the double cone $\mathscr{C}_{0}$. The thickness of this shell, of order $2 \alpha\left|z_{ \pm}\right|$, is proportional to $\left|z_{ \pm}\right|$and inversely proportional to $t$, a result first obtained by Makhortykh \& Rybak (1990).

Over a forcing period $2 \pi / \omega_{0}$, the average energy flux, given by

$$
\begin{aligned}
\boldsymbol{I}(\boldsymbol{x}, t) \sim & \frac{\rho_{0} N \mathscr{S}^{2} \cos \theta_{0}}{2^{11 / 2} \pi^{5 / 2} \alpha^{1 / 2}\left|x_{ \pm} z_{ \pm}\right|^{3 / 2}} \boldsymbol{e}_{z_{ \pm}}\left\{C\left[\left(\frac{2}{\pi} \frac{\left|x_{ \pm}\right|}{\alpha\left|z_{ \pm}\right|}\right)^{1 / 2}\right] \sin \left(\frac{\left|x_{ \pm}\right|}{\alpha\left|z_{ \pm}\right|}\right)\right. \\
& \left.-S\left[\left(\frac{2}{\pi} \frac{\left|x_{ \pm}\right|}{\alpha\left|z_{ \pm}\right|}\right)^{1 / 2}\right] \cos \left(\frac{\left|x_{ \pm}\right|}{\alpha\left|z_{ \pm}\right|}\right)\right\},
\end{aligned}
$$

is also modulated. As a consequence, the power output, given by

$$
P(\boldsymbol{x}, t) \sim \frac{\rho_{0} N \mathscr{S}^{2} \cos \theta_{0} \sin \theta_{0}}{8 \pi \alpha\left|z_{ \pm}\right|},
$$

is proportional to the time $t$ elapsed since the start-up and inversely proportional to the distance $\left|z_{ \pm}\right|$from the source. As for the Gaussian monopole, at constant strength $\mathscr{S}$, it varies as the inverse of the thickness of the shell.

The wave profiles are represented in figure 8 ; they are normalized by the centreline values given in table 1, consistent with the results of Makhortykh \& Rybak (1990) and Simakov (1993). They exhibit the same basic structure as for the Gaussian monopole, 


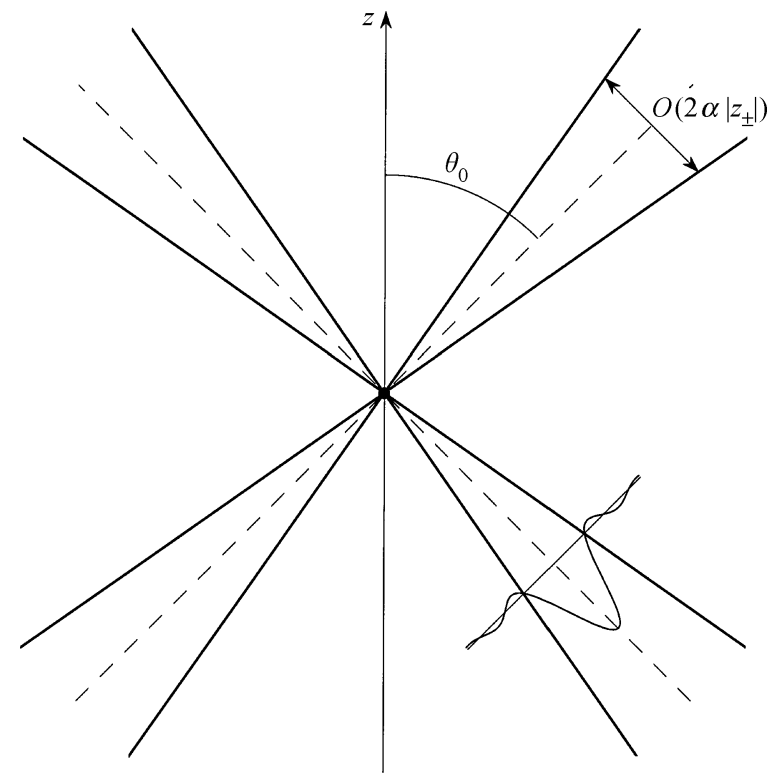

FIGURE 7. Wave beams from a source switched on impulsively at time $t=0$. The waves propagate inside a shell of thickness increasing as the distance $\left|z_{ \pm}\right|$from the source and decreasing as the inverse of the time $t$ elapsed since the start-up, around the double cone $\mathscr{C}_{0}$ shown dashed; $\alpha=1 /\left(N t \sin \theta_{0}\right)$. The wave energy flux, taken from figure $8(g)$, is represented on the lower beam.

except for the presence of oscillatory tails on either side of the centreline. These tails are associated with the separation, in the intermediate zone $\alpha\left|z_{ \pm}\right| \ll\left|x_{ \pm}\right| \ll\left|z_{ \pm}\right|$, of the two interfering components of the quasi-steady wave system: the steady waves of frequency $\omega_{0}$, given by (4.43)-(4.44), and the transient waves of frequency $N|z| / r$, given by

$$
\begin{aligned}
& p_{\mathrm{t}}(\boldsymbol{x}, t) \sim \frac{\rho_{0} N \mathscr{S}}{2^{5 / 2} \pi^{3 / 2}} \frac{\cos \theta_{0}}{x_{ \pm}} \frac{1}{\left(\omega_{0} t\right)^{1 / 2}} \exp \left[\mathrm{i}\left(\frac{x_{ \pm}}{\alpha\left|z_{ \pm}\right|}-\omega_{0} t-\frac{3 \pi}{4}\right)\right], \\
& \boldsymbol{u}_{\mathrm{t}}(\boldsymbol{x}, t) \sim \frac{\mathscr{S}}{2^{5 / 2} \pi^{3 / 2}} \frac{\boldsymbol{e}_{z_{ \pm}}}{x_{ \pm} z_{ \pm}}\left(\omega_{0} t\right)^{1 / 2} \exp \left[\mathrm{i}\left(\frac{x_{ \pm}}{\alpha\left|z_{ \pm}\right|}-\omega_{0} t-\frac{3 \pi}{4}\right)\right] .
\end{aligned}
$$

The transient pressure is $O\left[\left(\alpha\left|z_{ \pm}\right| /\left|x_{ \pm}\right|\right)^{1 / 2}\right]$ compared with the steady pressure, and thus negligible, while the transient velocity is $O\left[\left(\alpha\left|z_{ \pm}\right| /\left|x_{ \pm}\right|\right)^{-1 / 2}\right]$ compared with the steady velocity, and thus dominant; in both cases the complementary transient contributions of frequencies $-N|z| / r, N$ and $-N$ yield pressures and velocities which are at most $O\left(\left|x_{ \pm}\right| /\left|z_{ \pm}\right|\right)$compared with the dominant contribution, and thus negligible. Accordingly, to leading order in the intermediate zone the pressure is given by (4.43) and the velocity by (5.21), as is confirmed by figure 8 . To the same approximation, the asymptotic energy flux is not zero as for steady waves but

$$
\boldsymbol{I}(\boldsymbol{x}, t) \sim \pm \frac{\rho_{0} N \mathscr{S}^{2} \cos \theta_{0}}{64 \pi^{5 / 2} \alpha^{1 / 2}\left|x_{ \pm} z_{ \pm}\right|^{3 / 2}} \boldsymbol{e}_{z_{ \pm}} \sin \left(\frac{\left|x_{ \pm}\right|}{\alpha\left|z_{ \pm}\right|}-\frac{\pi}{4}\right) .
$$

This unexpected behaviour is an artefact of the point source formulation.

Away from the wave beams, in the outer zone where $N t \gg 1$ and $\left|x_{ \pm}\right| /\left|z_{ \pm}\right|=O(1)$, the wave function is given by (5.5). A similar separation occurs between steady waves of frequency $\omega_{0}$, with pressure and velocity given by (3.9)-(3.10), and transient gravity 

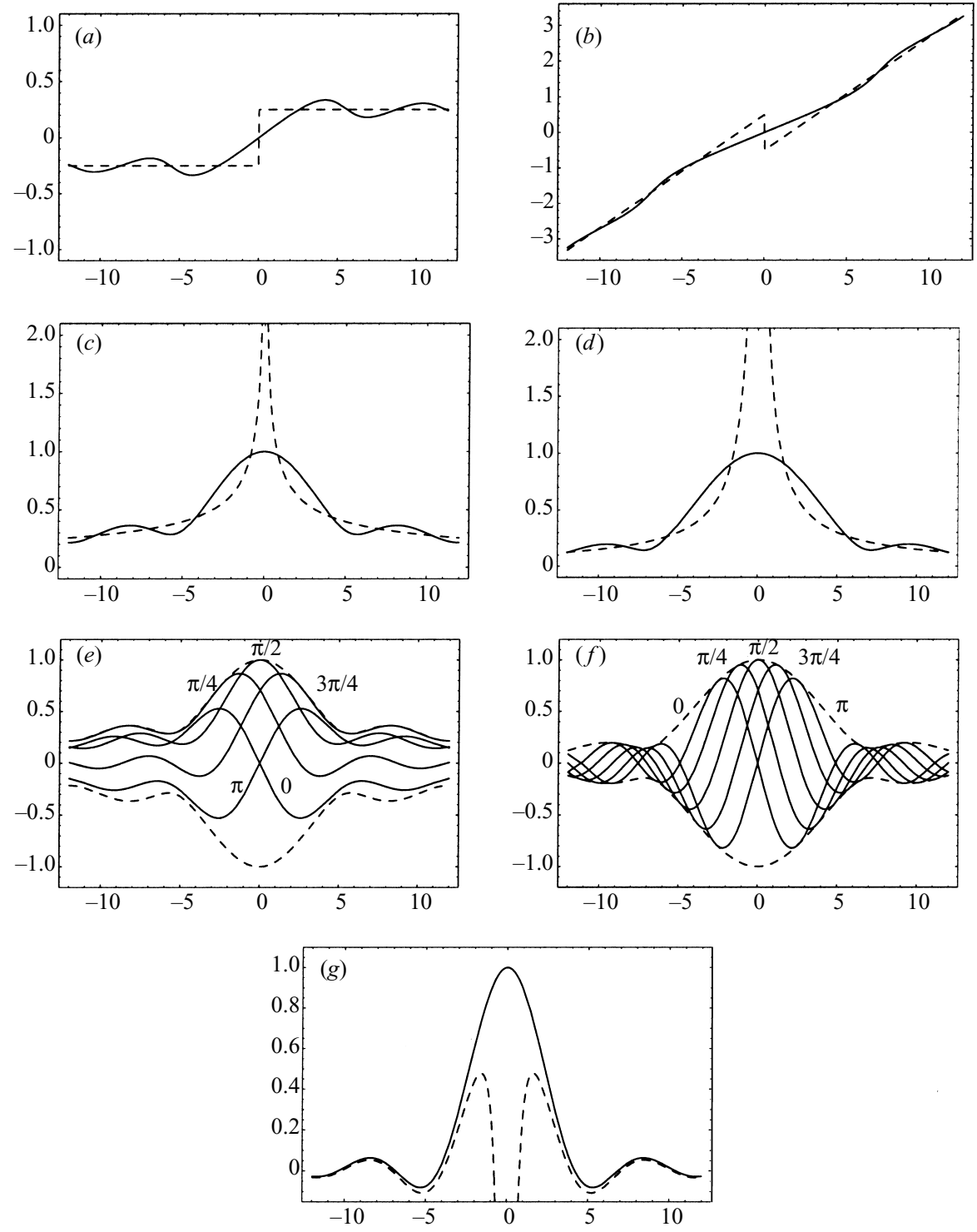

FIGURE 8. Waves from a monopole switched on impulsively at time $t=0$, as a function of $x_{ \pm} /\left(\alpha\left|z_{ \pm}\right|\right)$. The mode of representation is the same as in figure 5, with one exception: the dashed curves represent in $(a)$ and $(c)$ the steady asymptotic pressure (4.43), in $(b)$ and $(d)$ the transient asymptotic velocity (5.21), and in $(g)$ the asymptotic intensity (5.22) combining the two.

waves of frequencies $N|z| / r$ and $-N|z| / r$, with pressure and velocity given by $p_{\mathrm{t}}(\boldsymbol{x}, t) \sim \frac{\rho_{0} N^{2} \mathscr{S}}{2^{5 / 2} \pi^{3 / 2}} \frac{r_{\mathrm{h}}|z|}{r^{3}} \frac{1}{(N t|z| / r)^{1 / 2}}\left\{\frac{\exp [-\mathrm{i}(N t|z| / r-\pi / 4)]}{N|z| / r-\omega_{0}}+\frac{\exp [\mathrm{i}(N t|z| / r-\pi / 4)]}{N|z| / r+\omega_{0}}\right\}$,

$\boldsymbol{u}_{\mathrm{t}}(\boldsymbol{x}, t) \sim \frac{N \mathscr{S}}{2^{5 / 2} \pi^{3 / 2}} \frac{r_{\mathrm{h}} \boldsymbol{x}}{r^{4}}(N t|z| / r)^{1 / 2}\left\{\frac{\exp [-\mathrm{i}(N t|z| / r-\pi / 4)]}{N|z| / r-\omega_{0}}+\frac{\exp [\mathrm{i}(N t|z| / r-\pi / 4)]}{N|z| / r+\omega_{0}}\right\}$, 
the steady pressure (3.9) and the 'transient' velocity (5.24) being dominant. Compared with either of them the transient buoyancy oscillations at the frequencies $N$ and $-N$ are negligible, as seen in Voisin (1991).

\section{Waves in a viscous fluid}

We consider a steady monochromatic point monopole $q(\boldsymbol{x}, t)=\mathscr{S} \delta(\boldsymbol{x}) \exp \left(-\mathrm{i} \omega_{0} t\right)$, oscillating with strength $\mathscr{S} \exp \left(-\mathrm{i} \omega_{0} t\right)$ at the frequency $0<\omega_{0}<N$ in a fluid of kinematic viscosity $v$. In the literature, the wave equation for this source,

$$
\left[\left(\frac{\partial}{\partial t}-v \nabla^{2}\right) \frac{\partial}{\partial t} \nabla^{2}+N^{2} \nabla_{\mathrm{h}}^{2}\right] \chi(\boldsymbol{x}, t)=\mathscr{S} \delta(\boldsymbol{x}) \exp \left(-\mathrm{i} \omega_{0} t\right),
$$

has only been tackled in two dimensions: Thomas \& Stevenson (1972) solved it in real space, based on a boundary-layer approximation $\partial / \partial x_{ \pm} \gg \partial / \partial z_{ \pm}$, and Makarov et al. (1990) and Kistovich \& Chashechkin (1994) solved it in Fourier space, based on a low-viscosity approximation $v \kappa^{2} / N \ll 1$; in neither case was the domain of validity of the approximation specified. Ramachandra Rao \& Balan (1977) found numerically that the approximation amounts to a far-field assumption $N r^{2} / v \gg 1$. Lighthill (1978, $\S \S 4.7$ and 4.10) used a different approach, in two and three dimensions: rather than dealing directly with the wave equation (6.1), he analysed the effect of viscosity on an individual plane wave of wavenumber $k_{ \pm}$and incorporated the result - an exponential attenuation factor - into the spectral decomposition (4.14); implicitly an approximation was involved, that viscosity be small enough for inviscid internal waves to form, before being attenuated by viscosity as they propagate away from the source. Here we follow the Fourier-space route, paying special attention to specifying the underlying assumption.

\subsection{The Fourier-space way}

In Fourier space, the solution to (6.1) is the inverse transform

$$
\chi(\boldsymbol{x}, t)=\frac{\mathscr{S}}{8 \pi^{3}} \exp \left(-\mathrm{i} \omega_{0} t\right) \int \frac{\exp (\mathrm{i} \boldsymbol{k} \cdot \boldsymbol{x})}{\omega_{0}^{2} \kappa^{2}-N^{2} \kappa_{\mathrm{h}}^{2}+\mathrm{i} \omega_{0} \nu \kappa^{4}} \mathrm{~d}^{3} k,
$$

where the causal addition $+\mathrm{i} 0$ to the frequency, observed in (4.18) and (5.9), has been superseded by a viscous addition $+\mathrm{i} v \kappa^{2}$. In order to keep the inverse transform tractable we assume that viscosity is small enough for this addition to be a small perturbation. For this we postulate the existence of a cutoff wavenumber $\kappa_{0}$, to be determined later, such that viscous attenuation eliminates all wavenumbers significantly greater than $\kappa_{0}$; the low-viscosity assumption becomes $\nu \kappa_{0}^{2} / N \ll 1$, a condition of large Reynolds number.

We proceed, as before, in cylindrical coordinates $\left(\kappa_{\mathrm{h}}, \varphi_{k}, m\right)$, so that

$$
\begin{aligned}
\chi(\boldsymbol{x}, t) \sim \frac{\mathscr{S}}{8 \pi^{3} N^{2}} \exp \left(-\mathrm{i} \omega_{0} t\right) \int_{0}^{\infty} & \kappa_{\mathrm{h}} \mathrm{d} \kappa_{\mathrm{h}} \int_{0}^{2 \pi} \mathrm{d} \varphi_{k} \int_{-\infty}^{\infty} \mathrm{d} m \\
& \times \frac{\exp \left\{\mathrm{i}\left[\kappa_{\mathrm{h}} r_{\mathrm{h}} \cos \left(\varphi_{k}-\varphi\right)+m z\right]\right\}}{m^{2} \cos ^{2} \theta_{0}-\kappa_{\mathrm{h}}^{2} \sin ^{2} \theta_{0}+\mathrm{i} \nu \kappa^{4} / N \cos \theta_{0}} .
\end{aligned}
$$

In the complex $m$-plane the path of integration is the same as in figure 3 , except for an $O\left(v \kappa_{0}^{2} / N\right)$ displacement of the poles of the integrand, to the new positions $\kappa_{\mathrm{h}}\left[\tan \theta_{0}-\right.$ $\left.\mathrm{i} \nu \kappa_{\mathrm{h}}^{2} /\left(2 N \cos ^{4} \theta_{0} \sin \theta_{0}\right)\right]$ and $-\kappa_{\mathrm{h}}\left[\tan \theta_{0}-\mathrm{i} \nu \kappa_{\mathrm{h}}^{2} /\left(2 N \cos ^{4} \theta_{0} \sin \theta_{0}\right)\right]$. Deformation of this 
path selects the pole

$$
m=-\kappa_{\mathrm{h}}\left(\tan \theta_{0}-\mathrm{i} \frac{\nu \kappa_{\mathrm{h}}^{2}}{2 N \cos ^{4} \theta_{0} \sin \theta_{0}}\right) \operatorname{sign} z,
$$

with residue, in conical polar coordinates $\left(k_{ \pm}, \varphi_{k}, m_{ \pm}\right)$with $\pm=\operatorname{sign} z$,

$$
\begin{aligned}
\chi(\boldsymbol{x}, t) \sim-\mathrm{i} \frac{\mathscr{S}}{8 \pi^{2} N^{2} \sin \theta_{0}} \exp \left(-\mathrm{i} \omega_{0} t\right) \int_{0}^{\infty} \mathrm{d} k_{ \pm} & \exp \left(-\beta k_{ \pm}^{3}|z| / \cos \theta_{0}\right) \\
& \times \int_{0}^{2 \pi} \mathrm{d} \varphi_{k} \exp \left[\mathrm{i} k_{ \pm} \Phi\left(\varphi_{k} \mid \boldsymbol{x}\right)\right],
\end{aligned}
$$

where, in Lighthill's $(1978, \S 4.10)$ notation,

$$
\beta=\frac{v}{2 N \sin \theta_{0}},
$$

and where $\Phi\left(\varphi_{k} \mid \boldsymbol{x}\right)$ is defined by (4.22). The physical interpretation of this result is, as for an unsteady source, straightforward: since a plane internal wave of wavenumber $k_{ \pm}$is attenuated by viscosity at the rate $\beta k_{ \pm}^{3}$ per unit distance along rays (Lighthill $1978, \S \S 4.7$ and 4.10), at a given vertical level $|z|$ reached after travelling the distance $|z| / \cos \theta_{0}$ along the double cone $\mathscr{C}_{0}$, the amplitude of the waves has been multiplied by the exponential factor $\exp \left(-\beta k_{ \pm}^{3}|z| / \cos \theta_{0}\right)$. Accordingly, the cutoff wavenumber $\kappa_{0}$ is identified as $[N /(v r)]^{1 / 3}$, and the low-viscosity assumption $v \kappa_{0}^{2} / N \ll 1$ as the farfield approximation $N r^{2} / \nu \gg 1$, namely the approximation that the distance $r$ from the source be large compared with the thickness, of order $\left(v / \omega_{0}\right)^{1 / 2}$, of the boundary layer formed around this source as a consequence of its oscillation at the frequency $\omega_{0}$ in a fluid of kinematic viscosity $v$ (see e.g. Batchelor 1967, $\S \S 4.3,5.13$; Landau \& Lifshitz 1987, § 24).

Mathematically, the wave function (6.5) is of the same form (4.21) as for an extended source, with the attenuation factor $\mathscr{S} \exp \left(-\beta k_{ \pm}^{3}|z| / \cos \theta_{0}\right)$ playing the role of a spectrum. It is evaluated in the same way, yielding two distinct expansions, both of which involve the special functions $c_{\mu}(x)$ and $s_{\mu}(x)$ introduced by Thomas \& Stevenson (1972) and discussed in Appendix B. The first, uniform, expansion, valid wherever $N r^{2} / v \gg 1$, is

$$
\begin{aligned}
\chi(\boldsymbol{x}, t) \sim & \frac{\mathscr{S}}{4 \pi^{3 / 2} N^{2} \sin \theta_{0}}\left(\frac{\cos \theta_{0}}{\beta|z|}\right)^{1 / 6} \frac{\exp \left[-\mathrm{i}\left(\omega_{0} t+3 \pi / 4\right)\right]}{\left(x_{+}+x_{-}\right)^{1 / 2}}\left(c_{1 / 2}+\mathrm{i} s_{1 / 2}\right)\left[x_{ \pm}\left(\frac{\cos \theta_{0}}{\beta|z|}\right)^{1 / 3}\right] \\
& +\mathrm{i} \frac{\mathscr{S}}{4 \pi N^{2} \sin \theta_{0}} \frac{\exp \left(-\mathrm{i} \omega_{0} t\right)}{\left(x_{+}+x_{-}\right)^{1 / 2}\left(x_{ \pm}+\mathrm{i} 0\right)^{1 / 2}} \\
& -\mathrm{i} \frac{\mathscr{S}}{4 \pi N^{2} \sin \theta_{0}} \frac{\exp \left(-\mathrm{i} \omega_{0} t\right)}{\left(x_{+} x_{-}+\mathrm{i} 0\right)^{1 / 2}}
\end{aligned}
$$

where $|z| / \cos \theta_{0}=\left(x_{\mp}-x_{ \pm}\right) / \sin \left(2 \theta_{0}\right)$. The second, non-uniform, expansion divides the space into two zones, depending on whether the angular separation $\left|x_{+}\right| / r$ from $\mathscr{C}_{0}$ is finite and $O(1)$, or infinitesimal and $O\left[\left(v / N r^{2}\right)^{1 / 3}\right]$, respectively, as $N r^{2} / v \rightarrow \infty$. In the outer zone, corresponding to $z_{ \pm}^{2} / \beta \rightarrow \infty$ with $\left|x_{ \pm}\right| /\left|z_{ \pm}\right|=O(1)$, the first two terms of (6.7) combine into a higher-order term, and the third term, namely the inviscid wave function (3.7), becomes dominant; in the inner zone, corresponding to $z_{ \pm}^{2} / \beta \rightarrow \infty$ with $\left|x_{ \pm}\right| /\left(\beta\left|z_{ \pm}\right|\right)^{1 / 3}=O(1)$, the last two terms combine into another higher-order term, and 
the first term, given by

$$
\chi(\boldsymbol{x}, t) \sim \frac{\mathscr{S}}{2^{5 / 2} \pi^{3 / 2} N^{2} \cos ^{1 / 2} \theta_{0} \sin ^{3 / 2} \theta_{0}} \frac{\exp \left[-\mathrm{i}\left(\omega_{0} t+3 \pi / 4\right)\right]}{\beta^{1 / 6}\left|z_{ \pm}\right|^{2 / 3}}\left(c_{1 / 2}+\mathrm{i} s_{1 / 2}\right)\left[\frac{x_{ \pm}}{\left(\beta\left|z_{ \pm}\right|\right)^{1 / 3}}\right],
$$

becomes dominant.

In this way, the result of Lighthill $(1978, \S 4.10)$ is recovered, with two amendments: first, an implicit approximation is brought to the fore; secondly, when higher precision is looked for, and the uniform expansions (6.5) or (6.7) are used instead of (6.8), viscous attenuation is shown to involve the distance $|z| / \cos \theta_{0}=$ $\left|z_{ \pm} \mp x_{ \pm} \tan \theta_{0}\right|$ instead of $\left|z_{ \pm}\right|$, producing an asymmetry of the wave profile at any given $\left|z_{ \pm}\right|$.

\subsection{Structure of the wave field}

We investigate now the structure of the wave field. In the inner zone, where $\left|z_{ \pm}\right| \gg \beta^{1 / 2}$ and $\left|x_{ \pm}\right| /\left(\beta\left|z_{ \pm}\right|\right)^{1 / 3}=O(1)$, the wave function (6.8) gives, to leading order, by (4.36),

$$
\begin{gathered}
p(\boldsymbol{x}, t) \sim \frac{\rho_{0} N \mathscr{S}}{2^{5 / 2} \pi^{3 / 2}} \frac{\left(\cos \theta_{0} \sin \theta_{0}\right)^{1 / 2}}{\beta^{1 / 6}\left|z_{ \pm}\right|^{2 / 3}} \exp \left[-\mathrm{i}\left(\omega_{0} t+\frac{1}{4} \pi\right)\right]\left(c_{1 / 2}+\mathrm{i} s_{1 / 2}\right)\left[\frac{x_{ \pm}}{\left(\beta\left|z_{ \pm}\right|\right)^{1 / 3}}\right], \\
\boldsymbol{u}(\boldsymbol{x}, t) \sim \frac{\mathscr{S}}{2^{5 / 2} \pi^{3 / 2}} \frac{\cot ^{1 / 2} \theta_{0}}{\beta^{1 / 2} z_{ \pm}} \boldsymbol{e}_{z_{ \pm}} \exp \left[-\mathrm{i}\left(\omega_{0} t+\frac{1}{4} \pi\right)\right]\left(c_{3 / 2}+\mathrm{i} s_{3 / 2}\right)\left[\frac{x_{ \pm}}{\left(\beta\left|z_{ \pm}\right|\right)^{1 / 3}}\right] .
\end{gathered}
$$

Viscous effects manifest themselves through Thomas-Stevenson functions related to the Airy functions $\mathrm{Ai}(x)$ and $\operatorname{Bi}(x)$ by

$$
\begin{aligned}
& \left(c_{1 / 2}+\mathrm{i} s_{1 / 2}\right)(x)=\frac{\pi^{3 / 2}}{12^{1 / 6}}\left(\mathrm{Ai}^{2}+\mathrm{Bi}^{2}\right)\left(\mathrm{i} \frac{x}{12^{1 / 3}}\right), \\
& \left(c_{3 / 2}+\mathrm{i} s_{3 / 2}\right)(x)=\frac{\pi^{3 / 2}}{3^{1 / 2}}\left(\mathrm{Ai}^{\mathrm{Ai}^{\prime}}+\mathrm{Bi} \mathrm{Bi}^{\prime}\right)\left(\mathrm{i} \frac{x}{12^{1 / 3}}\right) .
\end{aligned}
$$

The associated energy flux is

$$
\boldsymbol{I}(\boldsymbol{x}) \sim \pm \frac{\rho_{0} N \mathscr{S}^{2} \cos \theta_{0}}{64 \pi^{3} \beta^{2 / 3}\left|z_{ \pm}\right|^{5 / 3}} \boldsymbol{e}_{z_{ \pm}}\left(c_{1 / 2} c_{3 / 2}+s_{1 / 2} s_{3 / 2}\right)\left[\frac{x_{ \pm}}{\left(\beta\left|z_{ \pm}\right|\right)^{1 / 3}}\right]
$$

and the power output is

$$
P(\boldsymbol{x}) \sim \frac{\rho_{0} N \mathscr{S}^{2} \cos \theta_{0} \sin \theta_{0}}{2^{7 / 3} 3^{3 / 2} \Gamma\left(\frac{2}{3}\right)\left(\beta\left|z_{ \pm}\right|\right)^{1 / 3}} .
$$

Wave beams are formed, shown in figure 9 , made of a conical shell of thickness of order $2\left(\beta\left|z_{ \pm}\right|\right)^{1 / 3}$ around the double cone $\mathscr{C}_{0}$, a result first obtained by Thomas \& Stevenson (1972) in two dimensions. As for the Gaussian or unsteady monopoles, at constant strength $\mathscr{S}$, the power output is inversely proportional to the thickness of the shell.

The wave profiles are represented in figure 10, together with the respective asymptotic expansions (4.43)-(4.44) in the intermediate zone $\left(\beta\left|z_{ \pm}\right|\right)^{1 / 3} \ll\left|x_{ \pm}\right| \ll\left|z_{ \pm}\right|$. The profiles exhibit the same structure as for the Gaussian monopole, with a supplementary tiny undulation on either side of the centreline. Away from this line, in the outer zone where $\left|z_{ \pm}\right| \gg \beta^{1 / 2}$ and $\left|x_{ \pm}\right| /\left|z_{ \pm}\right|=O(1)$, the pressure and velocity (3.9)-(3.10) are recovered. 


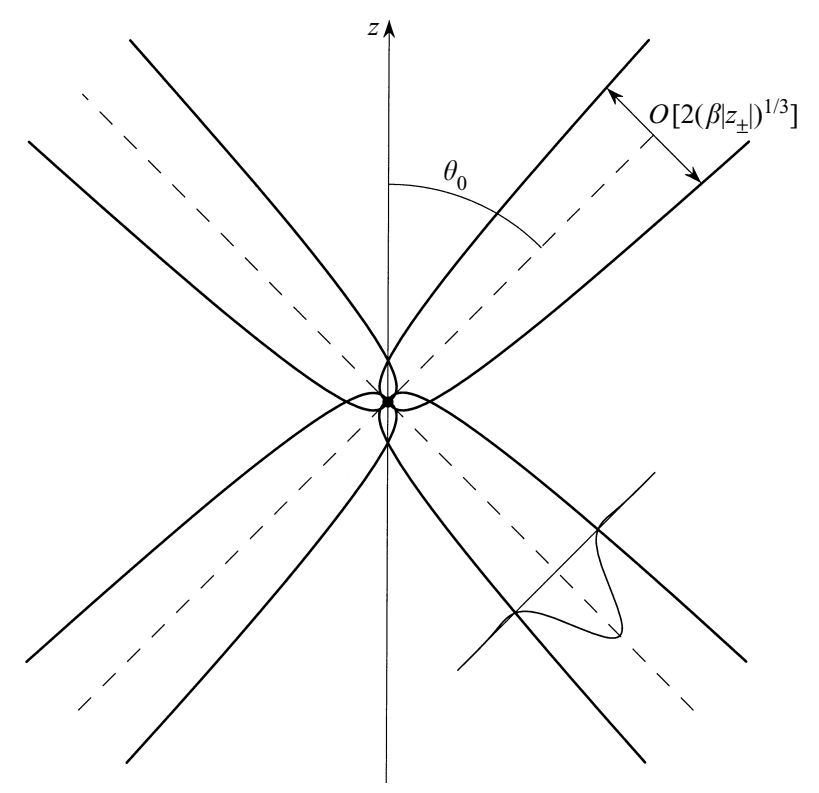

FIGURE 9. Wave beams from a source immersed in a fluid of kinematic viscosity $v$. The waves propagate inside a shell of thickness increasing as the one-third power of the distance $\left|z_{ \pm}\right|$ from the source, around the double cone $\mathscr{C}_{0}$ shown dashed; $\beta=v /\left(2 N \sin \theta_{0}\right)$. The wave energy flux, taken from figure $10(\mathrm{~g})$, is represented on the lower beam.

\section{Beam width}

Experimental measurement of internal waves in the literature involves generally, as in the reference work of Thomas \& Stevenson (1972) reproduced in figures 93 and 94 of Lighthill $(1978, \S 4.10)$, transverse plots such as successive waveforms accompanied by their envelope, and longitudinal plots such as the variations of the centreline amplitude and of the width of the wave beams with distance. In recent investigations, as allowed by the development of experimental and numerical techniques, twodimensional plots are also provided, such as density contours (Sutherland et al. 1999, 2000; Sutherland \& Linden 2002) and velocity vector fields (Javam et al. 2000).

In the preceding sections, some successive waveforms have been plotted together with their envelopes in figures 5, 6, 8 and 10, and the variations of the centreline amplitudes with distance have been given in table 1 . The width of the wave beams, however, has not been calculated, in that only order-of-magnitude estimates have been given, illustrated in figures 4,7 and 9 . In the literature the width of the beams is sometimes called wave width, or even wave length; in the following we shall call it beam width.

For an oscillating body, in the absence of unsteady or viscous effects, the energy flux is, in each beam, non-zero at distances from the centreline smaller than the radius of the body, and zero otherwise. Hence a natural definition of the beam width, implicit in the discussions of Hendershott (1969), Appleby \& Crighton (1987) and Voisin (1991) for a sphere, and Hurley $(1969,1972,1997)$, Appleby \& Crighton (1986) and Hurley \& Hood (2001) for a cylinder, is the diameter of the body. This definition corresponds also to the distance between the closest points, on either side of the centreline, where both the energy flux and the velocity are singular, as seen in figure 5 . 



FIGURE 10. Waves from a monopole immersed in a fluid of kinematic viscosity $v$, as a function of $x_{ \pm} /\left(\beta\left|z_{ \pm}\right|\right)^{1 / 3}$. The mode of representation is the same as in figure 5 .

When viscosity or unsteadiness comes into play, neither form of the definition holds any longer. Several incompatible generalizations have been introduced, summarized in figure 11. We will consider neither of them here, for different reasons: generalizations based on the odd part of the velocity profile - obtained here for $\omega_{0} t \equiv \pi / 4(\bmod 2 \pi)-$ rely on specific properties of this profile for a particular source, not observed for every source, such as the existence of zeros away from the centreline; generalizations based on the velocity amplitude are free from this limitation but introduce others, such as the arbitrary choice of a threshold fraction between 0 and 1 , a choice made necessary by the fact that the amplitude is, by definition, strictly positive. Moreover, the visible differences in figures 5, 6, 8 and 10 between the transverse rates of decay for the pressure on one hand, and the velocity on the other, imply that any definition based 


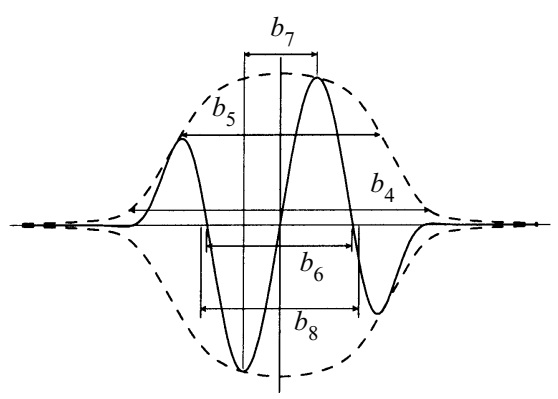

FIGURE 11. Definitions of the beam width in the literature: distance $b_{4}$ (respectively $b_{5}$ ) between the points where the velocity amplitude (dashed) is a fixed fraction 0.1 (respectively 0.6) of its central value (Thomas \& Stevenson 1972); distance $b_{6}$ between the first two zeros of the odd part of the velocity profile on either side of the central zero (Hurley \& Keady 1997; Javam et al. 2000; Hurley \& Hood 2001); distance $b_{7}$ between the first two extrema of the odd part of the velocity profile on either side of the central zero (Sutherland et al. 1999, 2000); distance $b_{8}$ equal to twice the standard deviation of the velocity amplitude profile (Sutherland \& Linden 2002). The velocity has been calculated in the two-dimensional conditions of figure $3 a$ of Javam et al. (2000), at Reynolds number $R e=25000$.

on the velocity is not fully appropriate for the pressure, and reciprocally that any definition based on the pressure is not fully appropriate for the velocity.

We propose three alternative definitions, based instead on the energy flux: physically, because of the interpretation of this flux as a characterization of the way that the source output is distributed through space by the waves; mathematically, because of the property that the outgoing flux is always positive at the centreline, takes both positive and negative values away from it, and has eventually a finite positive integral related by (4.41) to the power output.

The first definition, in which the shorthand $I\left(x_{ \pm}\right)= \pm I_{z_{ \pm}}(x, t)$ is introduced for the outgoing energy flux, generalizes the definition for oscillating bodies without unsteady or viscous effects: the width $b_{1}$ is the the distance, in each beam, between the first two zeros of $I\left(x_{ \pm}\right)$on either side of the central positive value, namely

$$
b_{1}=\left(\min _{x_{ \pm}>0}-\max _{x_{ \pm}<0}\right)\left\{x_{ \pm} \mid I\left(x_{ \pm}\right)=0\right\} .
$$

This definition takes advantage of the fact that, for all the sources investigated here save the Gaussian, the central region of outgoing flux is surrounded by regions of zero or incoming flux; it has already been used silently in figures 4, 7 and 9, when the beam contours and the flux profiles were superposed. The other two definitions take advantage of the fact that the outgoing flux has a finite positive integral, allowing the application of concepts inherited from signal processing (see e.g. Roddier 1978, chaps. 11 and 12): the equivalent width

$$
b_{2}=\frac{\int I\left(x_{ \pm}\right) \mathrm{d} x_{ \pm}}{I(0)}
$$

and twice the standard deviation, namely

$$
b_{3}=2\left\langle\left(x_{ \pm}-\left\langle x_{ \pm}\right\rangle\right)^{2}\right\rangle^{1 / 2},
$$




\begin{tabular}{lllll}
\hline Source & \multicolumn{1}{c}{$\ell$} & $b_{1} / \ell$ & $b_{2} / \ell$ & $b_{3} / \ell$ \\
Spherical & $2 a$ & 1 & $\frac{1}{2} \pi \approx 1.57$ & $\frac{1}{2^{1 / 2}} \approx 0.71$ \\
Gaussian & $2 a$ & $\infty$ & $\left(\frac{1}{2} \pi\right)^{1 / 2} \approx 1.25$ & 1 \\
Unsteady & $2 \alpha\left|z_{ \pm}\right|$ & 4.20 & $\frac{3}{4} \pi \approx 2.36$ & $\infty$ \\
Viscous & $2\left(\beta\left|z_{ \pm}\right|\right)^{1 / 3}$ & 4.25 & $\frac{3}{2} \Gamma\left(\frac{2}{3}\right) \approx 2.03$ & $\frac{3}{2^{2 / 3}}\left[\frac{\Gamma\left(\frac{2}{3}\right)}{\Gamma\left(\frac{1}{3}\right)}\right]^{1 / 2} \approx 1.34$
\end{tabular}

TABLE 2. Beam width, normalized by the length scale $\ell$ from figures 4,7 and 9 .

where, for any function $f\left(x_{ \pm}\right)$,

$$
\left\langle f\left(x_{ \pm}\right)\right\rangle=\frac{\int f\left(x_{ \pm}\right) I\left(x_{ \pm}\right) \mathrm{d} x_{ \pm}}{\int I\left(x_{ \pm}\right) \mathrm{d} x_{ \pm}} .
$$

The definitions $b_{1}, b_{2}$ and $b_{3}$ liken the profile of energy flux to that for an oscillating body, to a top-hat profile and to a Gaussian profile, respectively. The last of the three, $b_{3}$, has been used already by Sutherland $\&$ Linden (2002), with the energy flux replaced by the velocity amplitude.

The three definitions are applied in table 2 to the sources of the previous sections. None is fully satisfactory: $b_{1}$, whose determination, involving the solution of an implicit equation, is analytically the most complicated of the three, is infinite for the Gaussian monopole; $b_{3}$ is, for the pulsating sphere, smaller than the diameter of the sphere, and infinite for the unsteady monopole; $b_{2}$ is always finite, since the power output and the centreline energy flux are always finite, but its value differs by a factor of 2 or more from what we would expect from the visual inspection of some of the profiles in figures 5, 6, 8 and 10. Accordingly, the choice between the three definitions must consider other criteria, such as the ability, in a realistic wave field, to describe the transitions between the present limit states.

\section{Conclusions}

The three limit states of three-dimensional monochromatic linear internal wave fields have been considered in a uniformly stratified Boussinesq fluid otherwise at rest; they are associated with the size of the source, the start-up of the motion and the viscosity of the fluid, respectively. For all three states, the results of the previous investigators have been recovered and their domains of validity specified, leading to extended and improved results.

The analysis is asymptotic and relies on the assumptions of large distances $r$ from the source compared with its size $a$, large times $t$ elapsed since the start-up compared with the oscillation period $2 \pi / \omega_{0}$, and large distances $r$ compared with the viscous penetration depth $\left(2 v / \omega_{0}\right)^{1 / 2}$, respectively, with $v$ the kinematic viscosity. In each case the wave field organizes itself around the characteristic double cone $\mathscr{C}_{0}$ of vertical axis, apex at the source and semi-angle $\theta_{0}=\arccos \left(\omega_{0} / N\right)-$ the three-dimensional analogue of the two-dimensional St Andrew's Cross (Lighthill 1978, §4.4) - with $N$ the buoyancy frequency; it separates into two zones, respectively inner and outer, with boundary-layer structure. 


\begin{tabular}{cccccc}
\hline Source & Pressure & Velocity & Intensity & Power output & Beam width \\
Extended & $\frac{1}{\left|z_{ \pm}\right|^{1 / 2}}$ & $\frac{1}{\left|z_{ \pm}\right|^{1 / 2}}$ & $\frac{1}{\left|z_{ \pm}\right|}$ & const & const \\
Unsteady & $\frac{t^{n+1 / 2}}{\left|z_{ \pm}\right|^{n+1}}$ & $\frac{t^{n+3 / 2}}{\left|z_{ \pm}\right|^{n+2}}$ & $\frac{t^{2 n+2}}{\left|z_{ \pm}\right|^{2 n+3}}$ & $\frac{t^{2 n+1}}{\left|z_{ \pm}\right|^{2 n+1}}$ & $\frac{\left|z_{ \pm}\right|}{t}$ \\
Viscous & $\frac{1}{\left|z_{ \pm}\right|^{(n+2) / 3}}$ & $\frac{1}{\left|z_{ \pm}\right|^{(n+3) / 3}}$ & $\frac{1}{\left|z_{ \pm}\right|^{(2 n+5) / 3}}$ & $\frac{1}{\left|z_{ \pm}\right|^{(2 n+1) / 3}}$ & $\left|z_{ \pm}\right|^{1 / 3}$
\end{tabular}

TABLE 3. Variations of the waves with distance $\left|z_{+}\right|$and time $t$, for a source of multipolar order $2^{n}$. The pressure (amplitude), velocity (amplitude) and intensity are calculated at the centreline $x_{ \pm}=0$ of the beams.

The inner zone, a conical shell around $\mathscr{C}_{0}$, is the region where the formulae of the previous investigators hold and where the motion has indeed a wavy structure, with transverse phase propagation and longitudinal energy propagation. In the three limit states, in terms of the conical polar coordinates $\left(x_{ \pm}, \varphi, z_{ \pm}\right)$represented in figure 1 , the inner zone is defined mathematically by $\left|z_{ \pm}\right| / a \rightarrow \infty$ with $\left|x_{ \pm}\right| / a=O(1)$, as shown in figure $4, N t \rightarrow \infty$ with $\left|x_{+}\right| /\left(\alpha\left|z_{+}\right|\right)=O(1)$, as shown in figure 7 , and $\left|z_{+}\right| / \beta^{1 / 2} \rightarrow \infty$ with $\left|x_{ \pm}\right| /\left(\beta\left|z_{ \pm}\right|\right)^{1 / 3}=O(1)$, as shown in figure 9 , respectively, with $\alpha=1 /\left(N t \sin \theta_{0}\right)$ and $\beta=v /\left(2 N \sin \theta_{0}\right)$. The outer zone is defined by the same asymptotic limits, with $\left|x_{ \pm}\right| /\left|z_{ \pm}\right|=O(1)$, that is with finite angular separation from $\mathscr{C}_{0}$. In this zone the motion assumes the same structure as for a steady point source in an inviscid fluid, with algebraic decay of the amplitude with distance and no phase variation. Accordingly, the inner zone is identified as a double wave beam immersed in a fluid with no wave motion.

The calculations have been presented under the implicit assumption that the source is monopolar. Their adaptation to a source of arbitrary multipolar order $2^{n}$, such that the first non-zero moment of the source function $q_{0}(\boldsymbol{x})$ is of order $n$, is straightforward. Table 3 indicates the rates of variation of the wave properties with longitudinal distance $\left|z_{ \pm}\right|$and time $t$ inside the beams. The rate of decrease of the wave amplitude with distance is independent of the multipolar order for an extended source, and dependent on it otherwise. The beam width is always independent of the multipolar order, and the power output varies in inverse proportion to the $(2 n+1)$ th power of this width.

Whenever possible the calculations have been performed by two alternative procedures: one, in real space and real time, allowing an immediate grasp of the separation of the inner and outer zones; and another more intricate Fourierspace Fourier-time procedure. The greater mathematical complexity of the latter is counterbalanced by two advantages: first, the similarity between the treatments of the three limit states, setting the ground for their combined treatment; and secondly, the addition, to the non-uniform inner expansions (4.16), (5.6) and (6.8) and outer expansion (3.7), of uniform expansions (4.33), (5.13) and (6.7) valid in the two zones, and of alternative expressions (4.21), (5.12) and (6.5), exact for the source of finite size and asymptotic otherwise.

The latter expressions combine, in wavenumber space, two integrations along the wavenumber surface, a double cone $\mathscr{C}_{0}^{(k)}$ of vertical axis, apex at the origin and 
semi-angle $\pi / 2-\theta_{0}=\arcsin \left(\omega_{0} / N\right)$, defined by the dispersion relation. One integration, involving the radial coordinate $k_{ \pm}$, is already present in the inner expansion; it corresponds to superposing, at any given observation location, the contributions of all the wavenumber vectors associated with group velocity vectors pointing toward this location. The other integration, involving the azimuthal angle $\varphi_{k}$, corresponds to adding the contributions of all the other wavenumber vectors situated on the wavenumber surface; it is the three-dimensional analogue of the two-dimensional superposition of the left and right - or upper and lower, this is mathematically equivalent - wave beams, a superposition which the recent theoretical studies of Hurley (1997), Hurley \& Keady (1997) and Hurley \& Hood (2001) and experimental studies of Sutherland et al. (1999, 2000) and Sutherland \& Linden (2002) have proved mandatory close to the source. However, an important difference from the twodimensional case is the fact that the three-dimensional superposition does not involve a mere addition of the asymptotic expansions of the waves to be superposed, but a more intricate integration where the expression of each elementary wave of given polar angle $\varphi_{k}$ differs from the asymptotic expansion of the waves emitted with this angle $\varphi_{k}$.

Another unexpected result is the fact that, when the inner expansion is no longer valid, the distance $\left|z_{+}\right|$along the double cone $\mathscr{C}_{0}$, a distance involved in viscous attenuation and in cutoff due to the impulsive start-up, is replaced by the projection $|z| / \cos \theta_{0}=\left|z_{ \pm} \mp x_{ \pm} \tan \theta_{0}\right|$ of the vertical distance $|z|$ along the cone. Because of this, and of the superposition of different azimuthal waves, a transverse asymmetry with respect to $x_{ \pm}$is introduced into the wave profile at any given distance $\left|z_{ \pm}\right|$.

In addition to the usual characterizations of the waves in terms of the pressure and velocity, a new time-independent characterization has been proposed, in terms of the intensity or average energy flux, leading to three possible definitions of the beam width.

More detailed comparison with experiment is not possible at this stage, for lack of a known mathematical representation of the source of the waves - generally an oscillating body - as a source term in the wave equation.

This work has developed from coffee break discussions held at the 1st European Fluid Mechanics Conference in Cambridge, UK, in September 1991, with Professor David Crighton and Dr Frank Peters. It has been carried out partly at DAMTP, where it was funded in 1997 jointly by the Centre National de la Recherche Scientifique and the Royal Society through the European Science Exchange Programme, and in 1998 by the European Commission through Marie Curie fellowship No. 972653.

The benefit from conversations with Professor Yuli Chashechkin, Dr Bruce Sutherland and Mr Frans-Peter Lam, and from correspondence with Professor David Hurley and Drs Grant Keady and Sergey Simakov, is acknowledged. Professors Chantal Staquet, Claude Cambon and Dr Martin Galmiche are thanked for useful discussions on wave/vortex decomposition; Dr Theo Gerkema is thanked for bringing the Digital Library of Mathematical Functions to the author's attention, and Dr Michael Trott from Wolfram Research, Inc. for helping with Mathematica's evaluation of the Thomas-Stevenson functions. Professor Crighton contributed many useful discussions and helpful comments on an earlier draft of the paper. An anonymous referee is thanked for constructive criticism and insightful suggestions. Sadly, Professors Crighton and Hurley died in 2000. 


$$
\begin{array}{ll}
f(x) & \multicolumn{1}{c}{\int_{-\infty}^{\infty} f(x) \exp (-\mathrm{i} k x) \mathrm{d} x} \\
(x+\mathrm{i} 0)^{-\mu} \quad(\mu \neq-n) & 2 \pi \exp \left(-\mathrm{i} \frac{1}{2} \mu \pi\right) H(k) \frac{k^{\mu-1}}{\Gamma(\mu)} \\
x^{n} & 2 \pi \mathrm{i}^{n} \delta^{(n)}(k) \\
x^{n}\left[\psi(n+1)+\mathrm{i} \frac{1}{2} \pi-\ln (x+\mathrm{i} 0)\right] & 2 \pi \mathrm{i}^{-n} n ! \operatorname{Pf}\left[H(k) k^{-(n+1)}\right]
\end{array}
$$

TABlE 4. Some two-sided direct Fourier transforms, with $\mu$ complex and $n=0,1,2, \ldots$ a positive integer. The notation Pf indicates a finite part. $H(x)$ is the Heaviside step function, $\delta(x)$ the Dirac delta function and $\psi(x)$ the digamma function.

\section{Appendix A. Fourier transforms and regularization}

We define Fourier transforms, for any function $f(x)$, by the relations

$$
f(k)=\int f(x) \exp (-\mathrm{i} k x) \mathrm{d} x, \quad f(x)=\frac{1}{2 \pi} \int f(k) \exp (\mathrm{i} k x) \mathrm{d} k,
$$

and the extension of these to generalized functions. In the following, the extension is implicit even though the integral notation is used throughout.

Tables 4 and 5 gather some transforms of interest. The notation $x+\mathrm{i} 0=\lim _{\epsilon \rightarrow 0}(x+$ $\mathrm{i} \epsilon$ ), with $\epsilon>0$, indicates that the real variable $x$ is added a positive imaginary part which is later allowed to tend to 0 ; alternatively, cuts are inserted in the complex $x$-plane, extending from the real singularities of $f(x)$ vertically downwards. The transforms are either reproduced from Lighthill (1958, p. 43) and Gel'fand \& Shilov (1964, § II.2), or derived from Abramowitz \& Stegun (1972) and Gradshteyn \& Ryzhik (1994) at the values of the parameter $\mu$ for which the respective integrals converge then generalized by regularization.

\section{A.1. Regularization}

For functions with isolated algebraic singularities, of the form $H(k) k^{\mu-1}$ say, with $H(k)$ the Heaviside step function, regularization is described by Gel'fand \& Shilov (1964, $\S \S$ I.3-4) as the analytic continuation, in the complex $\mu$-plane, to all but negative integer values of $\mu$, and the Laurent expansion at these values, which are indeed simple poles with respect to the continuation, followed by selection of the first regular term of the expansion; a procedure equivalent, as pointed out by Schwartz (1966, $\S$ II.2), to taking Hadamard's finite part. In the following, we shall denote finite parts by a symbol Pf - read 'pseudo-function' - for a function and $f$ for an integral. Then, for negative integer $\mu=-n$ with $n=0,1,2, \ldots$, we have

$$
\operatorname{Pf}\left[H(k) k^{-(n+1)}\right]=\lim _{\mu \rightarrow-n} \frac{\partial}{\partial \mu}\left\{(\mu+n) \operatorname{Pf}\left[H(k) k^{\mu-1}\right]\right\} .
$$

This procedure, though, leaves one unsatisfactory issue: the finite part (A 2) does not satisfy the ordinary rules of addition, differentiation and multiplication by an infinitely differentiable function; in particular we have

$$
\frac{\mathrm{d}}{\mathrm{d} k} \operatorname{Pf}\left[H(k) k^{-n}\right]=-n \operatorname{Pf}\left[H(k) k^{-(n+1)}\right]+\frac{(-1)^{n}}{n !} \delta^{(n)}(k),
$$

with $\delta^{(n)}(k)$ the $n$th derivative of the Dirac delta function. Accordingly, Lighthill 

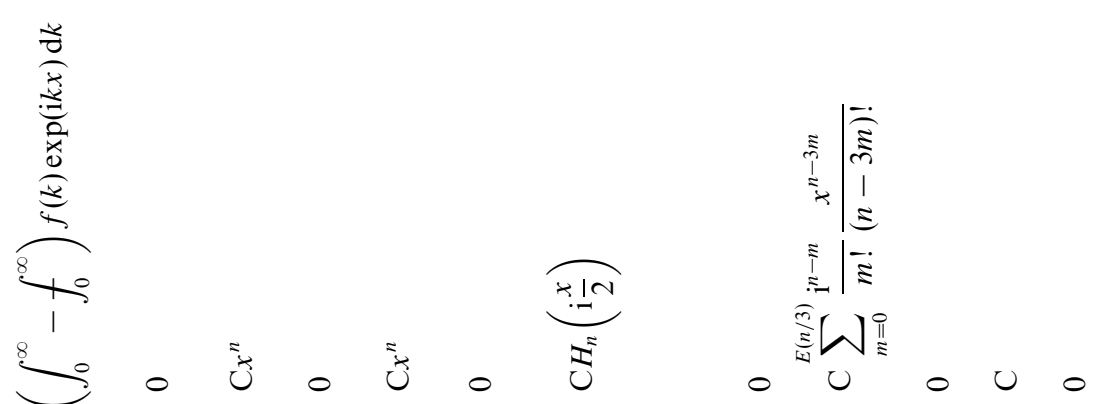

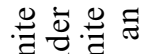

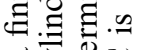
๙ 空包



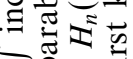
चृ 츠료욜 䛯芯

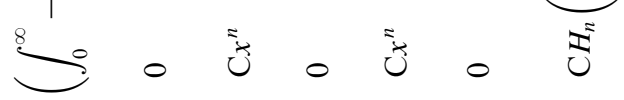

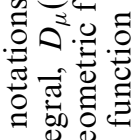
E.

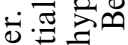

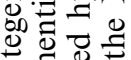

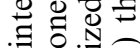
远芯 : 퐁

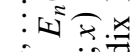
त $\tilde{1}$

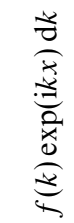

$\forall 10$ ने.气 II 킁효 ฮ 造

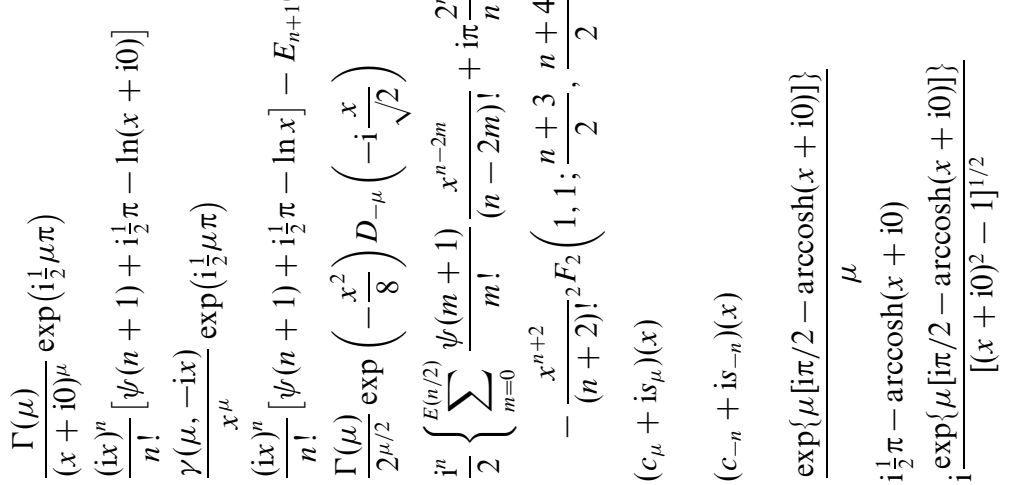

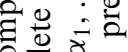
웅 ऽ 따웡 들 氖 उ.료.छ की 믈

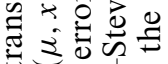

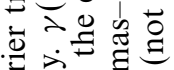

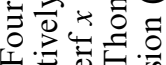

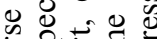

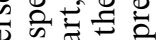

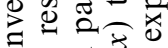

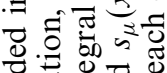

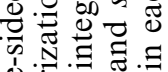

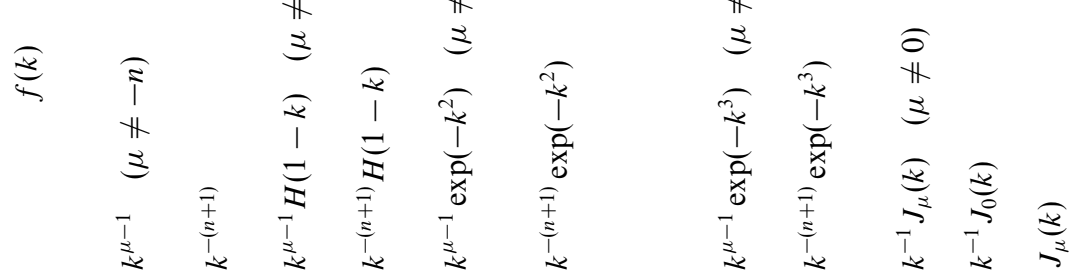

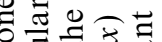
0.505 可记苛 คำ国

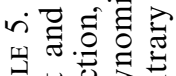

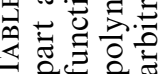


(1958, chap. 3) has introduced an amended definition of the generalized function $H(k) k^{-(n+1)}$, based on admitting an indeterminacy, in the same way as does the definition of indefinite integration: the symbol $H(k) k^{-1}$ stands for any generalized function $f(k)$ satisfying $k f(k)=H(k)$, and the symbol $H(k) k^{-(n+1)}$ for $(-1)^{n} / n$ ! times the $n$th derivative of any of these functions, so that

$$
H(k) k^{-(n+1)}=\operatorname{Pf}\left[H(k) k^{-(n+1)}\right]+\mathrm{C} \delta^{(n)}(k),
$$

with $\mathrm{C}$ an arbitrary constant. With this amendment, the function (A 4) satisfies the manipulation rules expected from a function called $H(k) k^{-(n+1)}$; in particular we have

$$
\frac{\mathrm{d}}{\mathrm{d} k}\left[H(k) k^{-n}\right]=-n H(k) k^{-(n+1)} .
$$

In the following, we shall call the finite part the original definition (A 2) and reserve the name regularization for the amended definition (A 4).

We extend these considerations to functions of the form $\phi(k) H(k) k^{\mu-1}$, with $\phi(k)$ infinitely differentiable. For $-n-1<\operatorname{Re} \mu \leqslant-n$ with $\mu \neq-n$ we write, as did Gel'fand \& Shilov (1964, § I.3.7),

$$
\begin{aligned}
\operatorname{Pf}\left[\phi(k) H(k) k^{\mu-1}\right]=\sum_{m=0}^{n} \frac{\phi^{(m)}(0)}{m !} \operatorname{Pf} & {\left[H(k) k^{\mu+m-1}\right] } \\
+ & {\left[\phi(k)-\sum_{m=0}^{n} \frac{\phi^{(m)}(0)}{m !} k^{m}\right] H(k) k^{\mu-1}, }
\end{aligned}
$$

where the first term on the right-hand side is of the type just discussed and the second term is locally summable, and

$$
\phi(k) H(k) k^{\mu-1}=\operatorname{Pf}\left[\phi(k) H(k) k^{\mu-1}\right] .
$$

For $\mu=-n$ we have

$$
\operatorname{Pf}\left[\phi(k) H(k) k^{-(n+1)}\right]=\lim _{\mu \rightarrow-n} \frac{\partial}{\partial \mu}\left\{(\mu+n) \operatorname{Pf}\left[\phi(k) H(k) k^{\mu-1}\right]\right\},
$$

and

$$
\phi(k) H(k) k^{-(n+1)}=\operatorname{Pf}\left[\phi(k) H(k) k^{-(n+1)}\right]+\mathrm{C} \sum_{m=0}^{n}(-1)^{m} \frac{\phi^{(m)}(0)}{m !} \frac{\delta^{(n-m)}(k)}{(n-m) !},
$$

with $\mathrm{C}$ an arbitrary constant (not the same as before).

We are interested in the Fourier transforms of these functions. Using table 5 we obtain, for $-n-1<\operatorname{Re} \mu \leqslant-n$ with $\mu \neq-n$, from (A 6)-(A 7),

$$
\begin{aligned}
f_{0}^{\infty} \phi(k) k^{\mu-1} \exp (\mathrm{i} k x) \mathrm{d} k & =\sum_{m=0}^{n} \frac{\phi^{(m)}(0)}{m !} \frac{\Gamma(\mu+m)}{(x+\mathrm{i} 0)^{\mu+m}} \exp \left[\mathrm{i} \frac{1}{2}(\mu+m) \pi\right] \\
& +\int_{0}^{\infty}\left[\phi(k)-\sum_{m=0}^{n} \frac{\phi^{(m)}(0)}{m !} k^{m}\right] k^{\mu-1} \exp (\mathrm{i} k x) \mathrm{d} k
\end{aligned}
$$

and

$$
\int_{0}^{\infty} \phi(k) k^{\mu-1} \exp (\mathrm{i} k x) \mathrm{d} k=f_{0}^{\infty} \phi(k) k^{\mu-1} \exp (\mathrm{i} k x) \mathrm{d} k .
$$


Similarly for $\mu=-n$ we have, from (A 8-(A 9),

$$
\begin{aligned}
& f_{0}^{\infty} \phi(k) k^{-(n+1)} \exp (\mathrm{i} k x) \mathrm{d} k=\sum_{m=0}^{n} \frac{\phi^{(m)}(0)}{m !} \frac{(\mathrm{i} x)^{n-m}}{(n-m) !}\left[\psi(n+1-m)+\mathrm{i} \frac{1}{2} \pi-\ln (x+\mathrm{i} 0)\right] \\
&+\int_{0}^{\infty}\left[\phi(k)-\sum_{m=0}^{n} \frac{\phi^{(m)}(0)}{m !} k^{m}\right] k^{-(n+1)} \exp (\mathrm{i} k x) \mathrm{d} k, \quad \text { (A 12) }
\end{aligned}
$$

where $\psi(x)$ is the digamma function, and

$$
\int_{0}^{\infty} \phi(k) k^{-(n+1)} \exp (\mathrm{i} k x) \mathrm{d} k=\int_{0}^{\infty} \phi(k) k^{-(n+1)} \exp (\mathrm{i} k x) \mathrm{d} k+\mathrm{C} \sum_{m=0}^{n} \frac{\phi^{(m)}(0)}{m !} \frac{(\mathrm{i} x)^{n-m}}{(n-m) !},
$$

where $\mathrm{C}$ is an arbitrary constant.

The function $H(k) k^{-1} J_{\mu}(k)$, with $J_{\mu}(k)$ the Bessel function of the first kind, is treated in the same way. Its only singularity in the complex $\mu$-plane is the simple pole $\mu=0$.

Algebraic functions $(x+\mathrm{i} 0)^{\mu-1}$ of the complex variable $x+\mathrm{i} 0$ are analytic functions of $\mu$ throughout the complex $\mu$-plane. They are related to the respective finite parts by

$$
(x+\mathrm{i} 0)^{\mu-1}=\operatorname{Pf}\left[H(x) x^{\mu-1}\right]+\exp [(\mathrm{i}(\mu-1) \pi)] \operatorname{Pf}\left[H(-x)(-x)^{\mu-1}\right],
$$

yielding for $\mu=-n$, by Laurent expansion, the Sokhotsky-Plemelj formula

$$
\frac{1}{(x+\mathrm{i} 0)^{n+1}}=\operatorname{Pf}\left(\frac{1}{x^{n+1}}\right)-\mathrm{i} \pi \frac{(-1)^{n}}{n !} \delta^{(n)}(x),
$$

discussed, for example, by Gel'fand \& Shilov (1964, § I.4.4).

\section{A.2. Derivation}

Of all the transforms given in tables 4 and 5 , only those corresponding to $\phi(k)=$ $\exp \left(-k^{2}\right)$ and $\exp \left(-k^{3}\right)$ require complementary explanations. The calculation of the latter is considered in Appendix B. The calculation of the former is straightforward for $\mu \neq-n$; for $\mu=-n$ we proceed as follows.

For $\operatorname{Re} \mu>0$ the series expansion of $\exp (\mathrm{i} k x)$ followed by term-by-term integration gives

$$
\int_{0}^{\infty} k^{\mu-1} \exp \left(-k^{2}\right) \exp (\mathrm{i} k x) \mathrm{d} k=\frac{1}{2} \sum_{m=0}^{\infty} \Gamma\left(\frac{\mu+m}{2}\right) \frac{(\mathrm{i} x)^{m}}{m !} .
$$

Analytic continuation to $\mu \neq-n$, followed by Laurent expansion at $\mu=-n$, yield

$$
\begin{array}{r}
f_{0}^{\infty} k^{-(n+1)} \exp \left(-k^{2}\right) \exp (\mathrm{i} k x) \mathrm{d} k=\frac{1}{2}\left[\mathrm{i}^{n} \sum_{m=0}^{E(n / 2)} \frac{\psi(m+1)}{m !} \frac{x^{n-2 m}}{(n-2 m) !}\right. \\
\left.+\sum_{m=0}^{\infty} \Gamma\left(\frac{m-n}{2}\right) \frac{(\mathrm{i} x)^{m}}{m !}\right],
\end{array}
$$

where $E(x)$ denotes the integral part and $\sum^{\prime}$ means that the values of $m$ such that $m-n$ is even negative are omitted. This sum $\sum^{\prime}$ is then separated into two parts. The first part, corresponding to odd $m-n$, combines a Hermite polynomial $H_{n}(x)$ with a repeated integral of the complementary error function erfc $x=1-\operatorname{erf} x$, with 
erf $x$ the error function, in the form

$$
\begin{gathered}
\sum_{p=-E[(n+1) / 2]}^{\infty} \Gamma\left(p+\frac{1}{2}\right) \frac{(\mathrm{i} x)^{n+2 p+1}}{(n+2 p+1) !}=\mathrm{i} \pi\left[\frac{1}{n !} H_{n}\left(\mathrm{i} \frac{x}{2}\right)-\int_{x}^{\infty} \cdots \int_{x}^{\infty} \operatorname{erfc}\left(\frac{x}{2}\right)(-\mathrm{i} \mathrm{d} x)^{n}\right] \\
=\mathrm{i} \pi \frac{(2 \mathrm{i})^{n}}{n !} \exp \left(-\frac{x^{2}}{4}\right) \frac{\mathrm{d}^{n}}{\mathrm{~d} x^{n}}\left[\exp \left(\frac{x^{2}}{4}\right) \operatorname{erf}\left(\frac{x}{2}\right)\right]
\end{gathered}
$$

see e.g. Abramowitz \& Stegun (1972, § 7.2 and chap. 22). The second part, corresponding to even strictly positive $m-n$, is a generalized hypergeometric function ${ }_{p} F_{q}\left(\alpha_{1}, \ldots, \alpha_{p} ; \beta_{1}, \ldots, \beta_{q} ; x\right)$ related to repeated integration of the imaginary error function erfi $x=-\mathrm{i} \operatorname{erf}(\mathrm{i} x)$, according to

$$
\begin{aligned}
\sum_{p=0}^{\infty} p ! \frac{(\mathrm{i} x)^{n+2 p+2}}{(n+2 p+2) !} & =\frac{(\mathrm{i} x)^{n+2}}{(n+2) !^{2}} F_{2}\left(1,1 ; \frac{n+3}{2}, \frac{n+4}{2} ;-\frac{x^{2}}{4}\right) \\
& =\mathrm{i} \pi^{1 / 2} \int_{0}^{x} \cdots \int_{0}^{x} \exp \left(-\frac{x^{2}}{4}\right) \operatorname{erfi}\left(\frac{x}{2}\right)(\mathrm{i} \mathrm{d} x)^{n+1}
\end{aligned}
$$

see e.g. Erdélyi et al. (1953, §9.9). Mathematica (Wolfram 1999) was instrumental in pointing out, at every particular $n$, the presence of the error and hypergeometric functions in the integral $f_{0}^{\infty} k-(n+1) \exp \left(-k^{2}\right) \exp (\mathrm{i} k x) \mathrm{d} k$, calculated from (A 12).

\section{Appendix B. Thomas-Stevenson functions}

\section{B.1. Definition and properties}

The functions $c_{\mu}(x)$ and $s_{\mu}(x)$ were introduced by Thomas $\&$ Stevenson (1972), based on the integral

$$
\left(c_{\mu}+\mathrm{i} s_{\mu}\right)(x)=\int_{0}^{\infty} k^{\mu-1} \exp \left(-k^{3}\right) \exp (\mathrm{i} k x) \mathrm{d} k,
$$

which converges for $\operatorname{Re} \mu>0$. In the original notation of Thomas \& Stevenson the index $\mu$ differs by 1 from that in (B 1 ).

We extend this definition by taking finite parts, applying (A 10) and (A 12) to obtain, for $-n-1<\operatorname{Re} \mu \leqslant-n$ with $\mu \neq-n$ and $n=0,1,2, \ldots$,

$$
\begin{aligned}
\left(c_{\mu}+\mathrm{i} s_{\mu}\right)(x)=\sum_{m=0}^{E(n / 3)} & \frac{\exp [\mathrm{i}(\mu+m) \pi / 2]}{m !} \frac{\Gamma(\mu+3 m)}{(x+\mathrm{i} 0)^{\mu+3 m}} \\
& +\int_{0}^{\infty} k^{\mu-1}\left[\exp \left(-k^{3}\right)-\sum_{m=0}^{E(n / 3)} \frac{\left(-k^{3}\right)^{m}}{m !}\right] \exp (\mathrm{i} k x) \mathrm{d} k
\end{aligned}
$$

and for $\mu=-n$,

$$
\begin{aligned}
\left(c_{-n}+\mathrm{i} s_{-n}\right)(x)= & \sum_{m=0}^{E(n / 3)} \frac{\mathrm{i}^{n-m}}{m !} \frac{x^{n-3 m}}{(n-3 m) !}\left[\psi(n+1-3 m)+\mathrm{i} \frac{1}{2} \pi-\ln (x+\mathrm{i} 0)\right] \\
& +\int_{0}^{\infty} k^{-(n+1)}\left[\exp \left(-k^{3}\right)-\sum_{m=0}^{E(n / 3)} \frac{\left(-k^{3}\right)^{m}}{m !}\right] \exp (\mathrm{i} k x) \mathrm{d} k .
\end{aligned}
$$



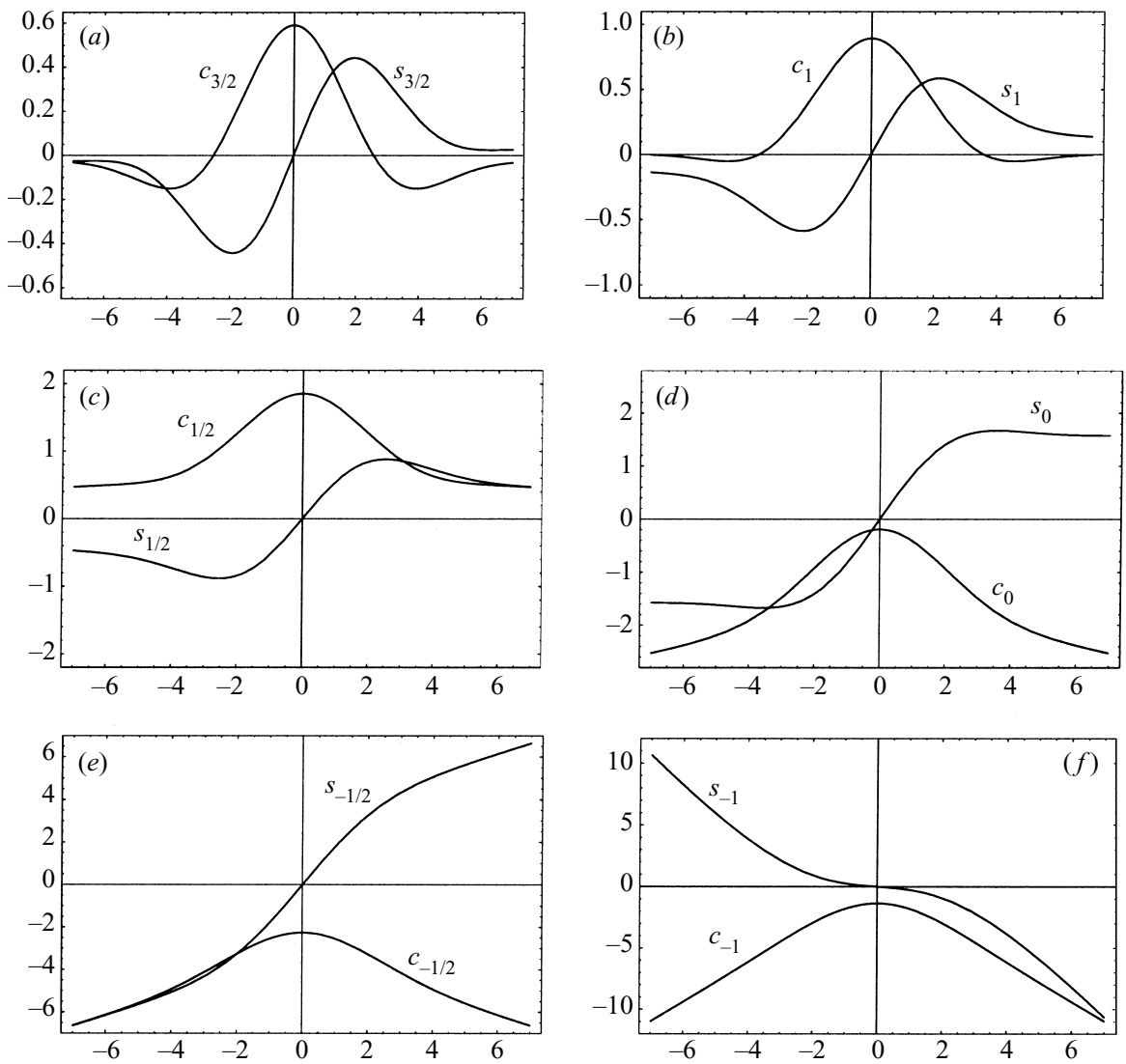

FIGURE 12. The functions $c_{\mu}(x)$ and $s_{\mu}(x)$ for some values of $\mu$.

The functions $c_{\mu}(x)$ and $s_{\mu}(x)$ are plotted in figure 12 for various values of $\mu$. They satisfy the differentiation rule

$$
\frac{\mathrm{d}}{\mathrm{d} x}\left(c_{\mu}+\mathrm{i} s_{\mu}\right)(x)=\mathrm{i}\left(c_{\mu+1}+\mathrm{i} s_{\mu+1}\right)(x)
$$

For $\mu \neq-n$ they also satisfy the recurrence relation

$$
3\left(c_{\mu+3}+\mathrm{i} s_{\mu+3}\right)(x)-\mathrm{i} x\left(c_{\mu+1}+\mathrm{i} s_{\mu+1}\right)(x)=\mu\left(c_{\mu}+\mathrm{i} s_{\mu}\right)(x),
$$

and have the series expansion, for $x \rightarrow 0$,

$$
\left(c_{\mu}+\mathrm{i} s_{\mu}\right)(x)=\frac{1}{3} \sum_{m=0}^{\infty} \Gamma\left(\frac{\mu+m}{3}\right) \frac{(\mathrm{i} x)^{m}}{m !},
$$

and the asymptotic expansion, for $x \rightarrow \infty$,

$$
\left(c_{\mu}+\mathrm{i} s_{\mu}\right)(x) \sim \sum_{m=0}^{\infty} \frac{\exp [\mathrm{i}(\mu+m) \pi / 2]}{m !} \frac{\Gamma(\mu+3 m)}{(x+\mathrm{i} 0)^{\mu+3 m}} .
$$


For $\mu=-n$ the recurrence relation becomes

$$
3\left(c_{3-n}+\mathrm{i} s_{3-n}\right)(x)-\mathrm{i} x\left(c_{1-n}+\mathrm{i} s_{1-n}\right)(x)=-n\left(c_{-n}+\mathrm{i} s_{-n}\right)(x)+\sum_{m=0}^{E(n / 3)} \frac{\mathrm{i}^{n-m}}{m !} \frac{x^{n-3 m}}{(n-3 m) !},
$$

the series expansion becomes

$$
\left(c_{-n}+\mathrm{i} s_{-n}\right)(x)=\frac{1}{3}\left[\sum_{m=0}^{E(n / 3)} \frac{\mathrm{i}^{n-m}}{m !} \frac{x^{n-3 m}}{(n-3 m) !} \psi(m+1)+\sum_{m=0}^{\infty} \Gamma\left(\frac{m-n}{3}\right) \frac{(\mathrm{i} x)^{m}}{m !}\right],
$$

where $\sum^{\prime}$ means that the values of $m$ such that $(m-n) / 3$ is a negative integer are omitted, and the asymptotic expansion becomes

$$
\begin{gathered}
\left(c_{-n}+\mathrm{i} s_{-n}\right)(x) \sim \sum_{m=0}^{E(n / 3)} \frac{\mathrm{i}^{n-m}}{m !} \frac{x^{n-3 m}}{(n-3 m) !}\left[\psi(n+1-3 m)+\mathrm{i} \frac{1}{2} \pi-\ln (x+\mathrm{i} 0)\right] \\
+\sum_{m=1+E(n / 3)}^{\infty} \frac{\mathrm{i}^{m-n}}{m !} \frac{(3 m-n-1) !}{x^{3 m-n}} .
\end{gathered}
$$

\section{B.2. Relation to hypergeometric functions}

On rearrangement of terms, each series expansion provides two expressions of the Thomas-Stevenson functions in terms of generalized hypergeometric functions, of imaginary and real arguments, respectively, the latter expression allowing the separation of $c_{\mu}(x)$ and $s_{\mu}(x)$.

For $\mu \neq-n$ we have from (B 6)

$$
\left(c_{\mu}+\mathrm{is}_{\mu}\right)(x)=\frac{1}{3} \sum_{m=0}^{2} \Gamma\left(\frac{\mu+m}{3}\right) \frac{(\mathrm{i} x)^{m}}{m !}{ }_{2} F_{3}\left(1, \frac{\mu+m}{3} ; \frac{m+1}{3}, \frac{m+2}{3}, \frac{m+3}{3} ;-\mathrm{i} \frac{x^{3}}{27}\right),
$$

and equivalently

$$
\begin{aligned}
\left(c_{\mu}+\mathrm{i} s_{\mu}\right)(x)= & \frac{1}{3} \sum_{m=0}^{5} \Gamma\left(\frac{\mu+m}{3}\right) \frac{(\mathrm{i} x)^{m}}{m !} \\
& \times{ }_{3} F_{6}\left(1, \frac{\mu+m}{6}, \frac{\mu+m+3}{6} ; \frac{m+1}{6}, \ldots, \frac{m+6}{6} ;-\frac{x^{6}}{11664}\right) .
\end{aligned}
$$

The latter result was first obtained by G. Keady (private communication) by applying Mathematica (Wolfram 1999) to the definition (B 1).

Similarly, for $\mu=-n$, we have from (B 9)

$$
\begin{aligned}
\left(c_{-n}\right. & \left.+\mathrm{i} s_{-n}\right)(x)=\frac{1}{3}\left[\sum_{m=0}^{E(n / 3)} \frac{\mathrm{i}^{n-m}}{m !} \frac{x^{n-3 m}}{(n-3 m) !} \psi(m+1)\right. \\
& +\frac{(\mathrm{i} x)^{n+3}}{(n+3) !^{2}} F_{3}\left(1,1 ; \frac{n+4}{3}, \frac{n+5}{3}, \frac{n+6}{3} ;-\mathrm{i} \frac{x^{3}}{27}\right) \\
& \left.+\sum_{m=0}^{2} \Gamma\left(\frac{m-n}{3}\right) \frac{(\mathrm{i} x)^{m}}{m !}{ }_{2} F_{3}\left(1, \frac{m-n}{3} ; \frac{m+1}{3}, \frac{m+2}{3}, \frac{m+3}{3} ;-\mathrm{i} \frac{x^{3}}{27}\right)\right],
\end{aligned}
$$


and equivalently

$$
\begin{aligned}
& \left(c_{-n}+\mathrm{i} s_{-n}\right)(x)=\frac{1}{3}\left[\sum_{m=0}^{E(n / 3)} \frac{\mathrm{i}^{n-m}}{m !} \frac{x^{n-3 m}}{(n-3 m) !} \psi(m+1)\right. \\
& \quad+\sum_{m=3,6} \frac{(\mathrm{i} x)^{n+m}}{(n+m) !}{ }_{3} F_{6}\left(1, \frac{m}{6}, \frac{m+3}{6} ; \frac{n+m+1}{6}, \ldots, \frac{n+m+6}{6} ;-\frac{x^{6}}{11664}\right) \\
& \left.\quad+\sum_{m=0}^{5} \Gamma\left(\frac{m-n}{3}\right) \frac{(\mathrm{i} x)^{m}}{m !}{ }_{3} F_{6}\left(1, \frac{m-n}{6}, \frac{m+3-n}{6} ; \frac{m+1}{6}, \ldots, \frac{m+6}{6} ;-\frac{x^{6}}{11664}\right)\right] .
\end{aligned}
$$

The latter result coincides, at every particular $n$, with Mathematica's (Wolfram 1999) evaluation of the definition (B 3).

\section{B.3. Relation to Airy functions}

For positive integer $\mu=n$, found in two-dimensional internal wave studies, the Thomas-Stevenson functions are related to the inhomogeneous Airy function $\mathrm{Hi}(x)$ introduced by Scorer (1950), and to a generalization $B_{1}(x,-n)$ of this function introduced by Drazin \& Reid (1981, Appendix). For a recent survey of Airy functions see chap. AI of the Digital Library of Mathematical Functions at the URL http://dlmf.nist.gov, and for an older survey see $\S 10.4$ of Abramowitz \& Stegun (1972).

For $\mu=1$, we have immediately

$$
\left(c_{1}+\mathrm{i} s_{1}\right)(x)=\frac{\pi}{3^{1 / 3}} \mathrm{Hi}\left(\mathrm{i} \frac{x}{3^{1 / 3}}\right),
$$

with $\operatorname{Hi}(x)=(1 / \pi) \int_{0}^{\infty} \exp \left(-k^{3} / 3+k x\right) \mathrm{d} k$, from which we deduce by integration

$$
\left(c_{0}+\mathrm{i} s_{0}\right)(x)=\pi \int_{0}^{\mathrm{i} x / 3^{1 / 3}} \operatorname{Hi}(t) \mathrm{d} t-\frac{1}{3} \gamma,
$$

with $\gamma$ the Euler constant. Alternatively, applying Hardy's generalization of the Airy function (Hardy 1910; Watson 1944, $\S 10.2-10.22$ ) to (B 1), or combining (B 11) with the expressions of the Bessel function $J_{v}(x)$ and Lommel function $s_{\mu, \nu}(x)$ as generalized hypergeometric functions (Watson 1944, $\S \S 4.4$ and 10.7), we obtain, in terms of the Lommel function $S_{\mu, v}(x)$,

$$
\left(c_{1}+\mathrm{i} s_{1}\right)(x)=\frac{2}{3^{3 / 2}} x^{1 / 2} \exp \left(-\mathrm{i} \frac{1}{4} \pi\right) S_{0,1 / 3}\left[2\left(\frac{x}{3}\right)^{3 / 2} \exp \left(-\mathrm{i} \frac{3}{4} \pi\right)\right],
$$

a result mentioned by Ramachandra Rao \& Balan (1977), or equivalently, in terms of the Anger function $\mathbf{J}_{v}(x)$,

$$
\left(c_{1}+\mathrm{i} s_{1}\right)(x)=\frac{2 \pi}{9} x^{1 / 2} \exp \left(-\mathrm{i} \frac{1}{4} \pi\right)\left(J_{-1 / 3}-J_{1 / 3}-\mathbf{J}_{-1 / 3}+\mathbf{J}_{1 / 3}\right)\left[2\left(\frac{x}{3}\right)^{3 / 2} \exp \left(-\mathrm{i} \frac{3}{4} \pi\right)\right] \text {. }
$$

More generally, we have, for strictly positive integer $\mu=n+1$,

$$
\left(c_{n+1}+\mathrm{i} s_{n+1}\right)(x)=\frac{1}{3^{\mu / 3}} B_{1}\left(\mathrm{i} \frac{x}{3^{1 / 3}},-n\right)
$$


with $B_{1}(x,-n)=\int_{0}^{\infty} k^{n} \exp \left(-k^{3} / 3+k x\right) \mathrm{d} k$. Separated expressions of $c_{1}(x)$ and $s_{1}(x)$ follow from (B 12); they involve linear combinations of the Kelvin functions ber $_{v}(x)$ and bei $_{v}(x)$ together with generalized hypergeometric functions.

For half-integer $\mu=1 / 2 \pm n$, found in three-dimensional internal wave studies, the Thomas-Stevenson functions are related to quadratic products of the Airy functions $\mathrm{Ai}(x)$ and $\operatorname{Bi}(x)$.

For $\mu=1 / 2$, we have immediately

$$
\left(c_{1 / 2}+\mathrm{i}_{1 / 2}\right)(x)=\frac{\pi^{3 / 2}}{12^{1 / 6}}\left(\mathrm{Ai}^{2}+\mathrm{Bi}^{2}\right)\left(\mathrm{i} \frac{x}{12^{1 / 3}}\right),
$$

as follows from $\left(\mathrm{Ai}^{2}+\mathrm{Bi}^{2}\right)(x)=\left(1 / \pi^{3 / 2}\right) \int_{0}^{\infty} k^{-1 / 2} \exp \left(-k^{3} / 12+k x\right) \mathrm{d} k$, an integral from the Digital Library of Mathematical Functions, or alternatively from the remark that $\left(c_{1 / 2}+\mathrm{i} s_{1 / 2}\right)(x)$ satisfies the differential equation of which $\mathrm{Ai}^{2}\left(\mathrm{i} x / 12^{1 / 3}\right)$, $\mathrm{Bi}^{2}\left(\mathrm{i} x / 12^{1 / 3}\right)$ and $\mathrm{Ai}\left(\mathrm{i} x / 12^{1 / 3}\right) \mathrm{Bi}\left(\mathrm{i} x / 12^{1 / 3}\right)$ are linearly independent solutions, followed by the use of (B 6) together with the series expansions of the Airy functions to determine the coefficients in front of each solution. By differentiation we obtain

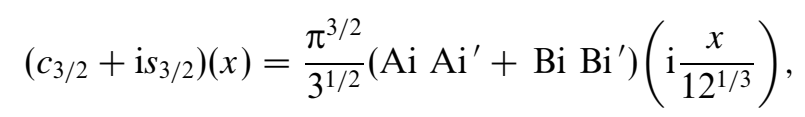

and by integration we have

$$
\left(c_{-1 / 2}+\mathrm{i}_{-1 / 2}\right)(x)=\mathrm{i} \frac{\pi^{3 / 2}}{12^{1 / 6}} x\left(\mathrm{Ai}^{2}+\mathrm{Bi}^{2}\right)\left(\mathrm{i} \frac{x}{12^{1 / 3}}\right)-12^{1 / 6} \pi^{3 / 2}\left(\mathrm{Ai}^{12}+\mathrm{Bi}^{12}\right)\left(\mathrm{i} \frac{x}{12^{1 / 3}}\right) .
$$

Separated expressions of $c_{1 / 2}(x)$ and $s_{1 / 2}(x)$ follow from (B 20); they involve quadratic products of the Kelvin functions $\operatorname{ber}_{v}(x)$ and bei $_{v}(x)$.

\section{Appendix C. Inviscid steady internal wave energy}

In an inviscid fluid, the linearized equations of motion (2.1)-(2.3) yield the energy conservation equation

$$
\frac{\partial}{\partial t}\left(\frac{1}{2} \rho_{0} \boldsymbol{u}^{2}+\frac{1}{2} \rho_{0} N^{2} \zeta^{2}\right)+\nabla \cdot(p \boldsymbol{u})=p q
$$

which, integrated over a fixed volume $V$ delimited by the surface $S$ of outward normal $\boldsymbol{n}$, becomes

$$
\frac{\mathrm{d}}{\mathrm{d} t} \int_{V}\left(\frac{1}{2} \rho_{0} \boldsymbol{u}^{2}+\frac{1}{2} \rho_{0} N^{2} \zeta^{2}\right) \mathrm{d}^{3} x+\oint_{S} p \boldsymbol{u} \cdot \boldsymbol{n} \mathrm{d}^{2} S=\int_{V} p q \mathrm{~d}^{3} x,
$$

and states that the variation within $V$ of the wave energy, $\rho_{0} \boldsymbol{u}^{2} / 2+\rho_{0} N^{2} \zeta^{2} / 2$ per unit volume, is equal to the sum of the incoming energy, $-p \boldsymbol{u} \cdot \boldsymbol{n}$ per unit area per unit time, transported across $S$, and of the work, $p q$ per unit volume per unit time, exerted by the source within $V$; here $\zeta$, defined by $w=\partial \zeta / \partial t$, is the vertical displacement. For a steady monochromatic source, after averaging over a period of oscillation, the first term of (C2), representing the rate of energy change within $V$, is zero; from the equality of the two remaining terms, we deduce that the power radiated across $S$ can be calculated either directly, as in $\S 4.3$, by integrating the wave energy flux over $S$, or indirectly by evaluating the rate of work produced within $V$. The latter procedure has been introduced by Gorodtsov \& Teodorovich $(1980,1981)$ for sources in uniform 
translation, then extended to sources with arbitrary time dependence (Gorodtsov \& Teodorovich 1983) and applied to monochromatic sources (Gorodtsov \& Teodorovich 1986). In the present Appendix we briefly outline their derivation.

The rate of work produced by a source $q$ generating pressure $p$ can be written, upon introducing Fourier transforms according to (2.15) and applying Parseval's theorem, as

$$
P(t)=\int p(\boldsymbol{x}, t) q(\boldsymbol{x}, t) \mathrm{d}^{3} x=\frac{1}{(2 \pi)^{3}} \int p(\boldsymbol{k}, t) q(-\boldsymbol{k}, t) \mathrm{d}^{3} k .
$$

Now, the relation $p(\boldsymbol{k}, \omega)=-\mathrm{i} \rho_{0} \omega\left(\omega^{2}-N^{2}\right) \chi(\boldsymbol{k}, \omega)$ between the pressure and the wave function, and that $\chi(\boldsymbol{k}, \omega)=q(\boldsymbol{k}, \omega) G(\boldsymbol{k}, \omega)$ between the wave function and the source, with $G(\boldsymbol{k}, \omega)$ the Fourier-transformed Green's function, imply

$$
p(\boldsymbol{k}, t)=-\mathrm{i} \frac{\rho_{0}}{2 \pi} \int \omega\left(\omega^{2}-N^{2}\right) q(\boldsymbol{k}, \omega) G(\boldsymbol{k}, \omega) \exp (-\mathrm{i} \omega t) \mathrm{d} \omega .
$$

By definition we also have

$$
q(\boldsymbol{k}, t)=\frac{1}{2 \pi} \int q(\boldsymbol{k}, \omega) \exp (-\mathrm{i} \omega t) \mathrm{d} \omega
$$

so that

$P(t)=-\mathrm{i} \frac{\rho_{0}}{(2 \pi)^{5}} \int \mathrm{d}^{3} k \int \mathrm{d} \omega \int \mathrm{d} \omega^{\prime} \omega\left(\omega^{2}-N^{2}\right) q(\boldsymbol{k}, \omega) q\left(-\boldsymbol{k}, \omega^{\prime}\right) G(\boldsymbol{k}, \omega) \exp \left[-\mathrm{i}\left(\omega+\omega^{\prime}\right) t\right]$.

This formula holds for any type of source $q(\boldsymbol{x}, t)$. We apply it to a monochromatic source $q_{0}(\boldsymbol{x}) \cos \left(\omega_{0} t\right)$, with $q_{0}(\boldsymbol{x})$ real. Upon time average, only the imaginary part, odd with respect to the frequency, of $G(\boldsymbol{k}, \omega)$ contributes to the integration. The combination of (2.19) with (A 15) gives

$$
G(\boldsymbol{k}, \omega)=\operatorname{Pf}\left(\frac{1}{\omega^{2} \kappa^{2}-N^{2} \kappa_{\mathrm{h}}^{2}}\right)-\mathrm{i} \pi \delta\left(\omega^{2} \kappa^{2}-N^{2} \kappa_{\mathrm{h}}^{2}\right) \operatorname{sign} \omega,
$$

and yields the average power

$$
P=\frac{\rho_{0} N^{3} \cos \theta_{0} \sin ^{2} \theta_{0}}{16 \pi^{2}} \int\left|q_{0}(\boldsymbol{k})\right|^{2} \delta\left(\omega_{0}^{2} \kappa^{2}-N^{2} \kappa_{\mathrm{h}}^{2}\right) \mathrm{d}^{3} k
$$

which involves only the wavenumber vectors situated on the wavenumber surface $\mathscr{C}_{0}^{(k)}$ prescribed by the dispersion relation $\omega_{0}^{2} \kappa^{2}-N^{2} \kappa_{\mathrm{h}}^{2}=0$. The wave power can be written either as an integral over $\mathscr{C}_{0}^{(k)}$, namely

$$
P=\frac{\rho_{0} N \sin \theta_{0}}{32 \pi^{2}} \int_{\mathscr{C}_{0}^{(k)}} \frac{\left|q_{0}(\boldsymbol{k})\right|^{2}}{\kappa} \mathrm{d}^{2} S_{k},
$$

consistently with the general formula (300) of Lighthill (1978, §4.9), or in conical polar coordinates $\left(k_{ \pm}, \varphi_{k}, m_{ \pm}\right)$as $(4.42)$.

\section{REFERENCES}

Abramowitz, M. \& Stegun, I. A. (Ed.) 1972 Handbook of Mathematical Functions, 10th printing. Dover.

Appleby, J. C. \& Crighton, D. G. 1986 Non-Boussinesq effects in the diffraction of internal waves from an oscillating cylinder. Q. J. Mech. Appl. Maths 39, 209-231. 
Appleby, J. C. \& Crighton, D. G. 1987 Internal gravity waves generated by oscillations of a sphere. J. Fluid Mech. 183, 439-450.

Batchelor, G. K. 1967 An Introduction to Fluid Dynamics. Cambridge University Press.

Chang, W. L. \& Stevenson, T. N. 1975 Internal waves in a viscous atmosphere. J. Fluid Mech. 72, 773-786.

Chashechin, Yu. D. \& Kistovich, YU. V. 1997 Generation of monochromatic internal waves: an exact solution and the force-source model. Phys. Dokl. 42, 377-380. [Transl. from Dokl. Akad. Nauk 355, 54-57.]

Chashechinin, Yu. D., Kistovich, Yu. V. \& IL’inykh, Yu. S. 2000 Experimental study of the generation of periodic internal waves by the boundary layer on a rotating disk. Dokl. Phys. 45, 627-631. [Transl. from Dokl. Akad. Nauk 375, 338-342.]

Chashechin, Yu. D., Kistovich, Yu. V. \& Smirnov, S. A. 2001 Linear generation theory of $2 \mathrm{D}$ and $3 \mathrm{D}$ periodic internal waves in a viscous stratified fluid. Environmetrics 12, $57-80$.

Crighton, D. G. 1986 Generation of internal gravity waves by turbulence. (Unpublished report).

Dalziel, S. B., Hughes, G. O. \& Sutherland, B. R. 2000 Whole-field density measurements by 'synthetic schlieren'. Exps. Fluids 28, 322-335.

Drazin, P. G. \& ReID, W. H. 1981 Hydrodynamic Stability. Cambridge University Press.

Erdélyi, A., Magnus, W., Oberhettinger, F. \& Tricomi, F. G. 1953 Higher Transcendental Functions, vol. 2. McGraw-Hill.

ERMANYUK, E. V. 2000 The use of impulse response functions for evaluation of added mass and damping coefficient of a circular cylinder oscillating in linearly stratified fluid. Exps. Fluids 28, $152-159$.

ERmanyuK, E. V. 2002 The rule of affine similitude for the force coefficients of a body oscillating in a uniformly stratified fluid. Exps. Fluids 32, 242-251.

Ermanyuk, E. V. \& Gavrilov, N. V. $2002 a$ Force on a body in a continuously stratified fluid. Part 1. Circular cylinder. J. Fluid Mech. 451, 421-443.

Ermanyuk, E. V. \& Gavrilov, N. V. $2002 b$ Oscillations of cylinders in a linearly stratified fluid. J. Appl. Mech. Tech. Phys. 43, 503-511. [Transl. from Prikl. Mekh. Tekh. Fiz. 43(4), 15-26.]

Ermanyuk, E. V. \& Gavrilov, N. V. 2003 Force on a body in a continuously stratified fluid. Part 2. Sphere. J. Fluid Mech. 494, 33-50.

FLYNN, M. R., ONU, K. \& SUTHERLAND, B. R. 2003 Internal wave excitation by a vertically oscillating sphere. J. Fluid Mech. 494, 65-93.

Gavrilov, N. V. \& ErmanyuK, E. V. 1997 Internal waves generated by circular translational motion of a cylinder in a linearly stratified fluid. J. Appl. Mech. Tech. Phys. 38, 224-227. [Transl. from Prikl. Mekh. Tekh. Fiz. 38(2), 64-67.]

Gel'fand, I. M. \& Shilov, G. E. 1964 Generalized Functions. Volume 1. Properties and Operations. Academic.

Gordon, D. \& Stevenson, T. N. 1972 Viscous effects in a vertically propagating internal wave. J. Fluid Mech. 56, 629-639.

Gorodtsov, V. A. \& Teodorovich, E. V. 1980 On the generation of internal waves in the presence of uniform straight-line motion of local and nonlocal sources. Izv. Atmos. Ocean. Phys. 16, 699-704. [Transl. from Izv. Akad. Nauk SSSR Fiz. Atmos. Okeana 16, 954-961.]

Gorodtsov, V. A. \& TeOdorovich, E. V. 1981 Two-dimensional problem for internal waves generated by moving singular sources. Fluid Dyn. 16, 219-224. [Transl. from Izv. Akad. Nauk SSSR Mekh. Zhidk. Gaza 16(2), 77-83.]

Gorodtsov, V. A. \& Teodorovich, E. V. 1982 Study of internal waves in the case of rapid horizontal motion of cylinders and spheres. Fluid Dyn. 17, 893-898. [Transl. from Izv. Akad. Nauk SSSR Mekh. Zhidk. Gaza 17(6), 94-100.]

Gorodtsov, V. A. \& TeOdorovich, E. V. 1983 Radiation of internal waves by periodically moving sources. J. Appl. Mech. Tech. Phys. 24, 521-526. [Transl. from Zh. Prikl. Mekh. Tekh. Fiz. 24(4), 81-87.]

Gorodtsov, V. A. \& TEOdOROvich, E. V. 1986 Energy characteristics of harmonic internal wave generators. J. Appl. Mech. Tech. Phys. 27, 523-529. [Transl. from Zh. Prikl. Mekh. Tekh. Fiz. 27(4), 53-59.] 
GöRTLER, H. 1943 Über eine Schwingungserscheinung in Flüssigkeiten mit stabiler Dichteschichtung. Z. angew. Math. Mech. 23, 65-71.

Gradshteyn, I. S. \& RyzhiK, I. M. 1994 Table of Integrals, Series, and Products, 5th edn. Academic.

HaRdY, G. H. 1910 On certain definite integrals considered by Airy and by Stokes. Q. J. Pure Appl. Maths 41, 226-240.

HART, R. W. 1981 Generalized scalar potentials for linearized three-dimensional flows with vorticity. Phys. Fluids 24, 1418-1420.

Hendershott, M. C. 1969 Impulsively started oscillations in a rotating stratified fluid. J. Fluid Mech. 36, 513-527.

Hinch, E. J. 1991 Perturbation Methods. Cambridge University Press.

Holm, D. D. \& KimuRA, Y. 1991 Zero-helicity Lagrangian kinematics of three-dimensional advection. Phys. Fluids A 3, 1033-1038.

Hurley, D. G. 1969 The emission of internal waves by vibrating cylinders. J. Fluid Mech. 36, $657-672$.

HuRley, D. G. 1972 A general method for solving steady-state internal gravity wave problems. J. Fluid Mech. 56, 721-740.

Hurley, D. G. 1997 The generation of internal waves by vibrating elliptic cylinders. Part 1. Inviscid solution. J. Fluid Mech. 351, 105-118.

Hurley, D. G. \& Hood, M. J. 2001 The generation of internal waves by vibrating elliptic cylinders. Part 3. Angular oscillations and comparison of theory with recent experimental observations. J. Fluid Mech. 433, 61-75.

Hurley, D. G. \& KeADY, G. 1997 The generation of internal waves by vibrating elliptic cylinders. Part 2. Approximate viscous solution. J. Fluid Mech. 351, 119-138.

ILINYKh, YU. S., Kistovich, YU. V. \& Chashechkin, YU. D. 1999 Comparison of an exact solution to a certain problem of periodic internal wave generation with experiment. Izv. Atmos. Ocean. Phys. 35, 589-594. [Transl. from Izv. Akad. Nauk Fiz. Atmos. Okeana 35, 649-655.]

IL'Inykh, Yu. S., Smirnov, S. A. \& Chashechinin, Yu. D. 1999 Excitation of harmonic internal waves in a viscous continuously stratified liquid. Fluid Dyn. 34, 890-895. [Transl. from Izv. Akad. Nauk Mekh. Zhidk. Gaza 34(6), 141-148.]

Ivanov, A. V. 1989 Generation of internal waves by an oscillating source. Izv. Atmos. Ocean. Phys. 25, 61-64. [Transl. from Izv. Akad. Nauk SSSR Fiz. Atmos. Okeana 25, 84-89.]

JAVAM, A., Imberger, J. \& ARMfield, S. W. 2000 Numerical study of internal wave-wave interactions in a stratified fluid. J. Fluid Mech. 415, 65-87.

Kistovich, A. V., Neklyudov, V. I. \& Chashechkin, YU. D. 1990 Nonlinear two-dimensional internal waves generated by a periodically moving source in an exponentially stratified medium. Izv. Atmos. Ocean. Phys. 26, 771-776. [Transl. from Izv. Akad. Nauk SSSR Fiz. Atmos. Okeana 26, 1051-1060.]

Kistovich, Yu. V. \& Chashechin, Yu. D. 1994 Reflection of packets of internal waves from a rigid plane in a viscous fluid. Izv. Atmos. Ocean. Phys. 30, 718-724. [Transl. from Izv. Akad. Nauk Fiz. Atmos. Okeana 30, 752-758.]

Kistovich, Yu. V. \& Chashechinin, Yu. D. $1999 a$ Generation of monochromatic internal waves in a viscous fluid. J. Appl. Mech. Tech. Phys. 40, 1020-1028. [Transl. from Prikl. Mekh. Tekh. Fiz. 40(6), 31-40.]

Kistovich, Yu. V. \& Chashechkin, YU. D. $1999 b$ An exact solution of a linearized problem of the radiation of monochromatic internal waves in a viscous fluid. J. Appl. Maths Mech. 63, 587-594. [Transl. from Prikl. Mat. Mekh. 63, 611-619.]

Kistovich, YU. V. \& ChashechKin, YU. D. 1999c Nonlinear generation of periodic internal waves by a boundary-layer flow around a rotating axially symmetric body. Dokl. Phys. 44, 573-576. [Transl. from Dokl. Akad. Nauk 367, 636-639.]

Kistovich, YU. V. \& Chashechinin, Yu. D. 2001a Some exactly solvable problems of the radiation of three-dimensional periodic internal waves. J. Appl. Mech. Tech. Phys. 42, 228-236. [Transl. from Prikl. Mekh. Tekh. Fiz. 42(2), 52-61.]

Kistovich, Yu. V. \& Chashechin, YU. D. $2001 b$ Generation of periodic internal waves by an oscillating strip of finite width. Dokl. Phys. 46, 667-671. [Transl. from Dokl. Akad. Nauk 380, 51-55.]

Kistovich, Yu. V. \& Chashechkin, Yu. D. 2002 A new mechanism of the nonlinear generation of internal waves. Dokl. Phys. 47, 163-167. [Transl. from Dokl. Akad. Nauk 382, 772-776.] 
Krishna, D. V. \& Sarma, L. V. K. V. 1969 Motion of an axisymmetric body in a rotating stratified fluid confined between two parallel planes. J. Fluid Mech. 38, 833-842.

LAI, R. Y. S. \& LEE, C.-M. 1981 Added mass of a spheroid oscillating in a linearly stratified fluid. Intl J. Engng Sci. 19, 1411-1420.

Landau, L. D. \& Lifshitz, E. M. 1987 Fluid Mechanics, 2nd edn. Butterworth-Heinemann.

Laws, P., Peat, K. S. \& Stevenson, T. N. 1982 An interferometer to study density stratified flows. J. Phys. E Sci. Instrum. 15, 1327-1331.

Lighthill, M. J. 1958 Introduction to Fourier Analysis and Generalised Functions. Cambridge University Press.

Lighthill, J. 1978 Waves in Fluids. Cambridge University Press.

Lighthill, J. 1990 Emendations to a proof in the general three-dimensional theory of oscillating sources of waves. Proc. R. Soc. Lond. A 427, 31-42.

Lighthill, J. 1996 Internal waves and related initial-value problems. Dyn. Atmos. Oceans 23, $3-17$.

Mclaren, T. I., Pierce, A. D., Fohl, T. \& Murphy, B. L. 1973 An investigation of internal gravity waves generated by a buoyantly rising fluid in a stratified medium. J. Fluid Mech. 57, $229-240$.

Makarov, S. A., Neklyudov, V. I. \& Chashechin, Yu. D. 1990 Spatial structure of twodimensional monochromatic internal-wave beams in an exponentially stratified liquid. Izv. Atmos. Ocean. Phys. 26, 548-554. [Transl. from Izv. Akad. Nauk SSSR Fiz. Atmos. Okeana 26, 744-754.]

Maкhortykн, S. A. \& Rybak, S. A. 1990 Effect of the near field of a point source on the generation of internal waves. Izv. Atmos. Ocean. Phys. 26, 194-198. [Transl. from Izv. Akad. Nauk SSSR Fiz. Atmos. Okeana 26, 269-276.]

MowbraY, D. E. \& RARITY, B. S. H. 1967 A theoretical and experimental investigation of the phase configuration of internal waves of small amplitude in a density stratified liquid. J. Fluid Mech. 28, $1-16$.

Onu, K., Flynn, M. R. \& Sutherland, B. R. 2003 Schlieren measurement of axisymmetric internal wave amplitudes. Exps. Fluids 35, 24-31.

Peters, F. 1985 Schlieren interferometry applied to a gravity wave in a density-stratified liquid. Exps. Fluids 3, 261-269.

Ramachandra RaO, A. \& Balan, K. C. 1977 Effect of viscosity on internal waves from a source in a wall. Proc. Indian Acad. Sci. A 85, 351-366.

Riley, J. J. \& Lelong, M.-P. 2000 Fluid motions in the presence of strong stable stratification. Annu. Rev. Fluid Mech. 32, 613-657.

RODDIER, F. 1978 Distributions et Transformation de Fourier. McGraw-Hill.

SARMA, L. V. K. V. \& KrishnA, D. V. 1972 Oscillation of axisymmetric bodies in a stratified fluid. Zastosow. Matem. 13, 109-121.

Schwartz, L. 1966 Théorie des Distributions, 2nd edn. Hermann.

SCORER, R. S. 1950 Numerical evaluation of integrals of the form $I=\int_{x_{1}}^{x_{2}} f(x) \mathrm{e}^{\mathrm{i} \phi(x)} \mathrm{d} x$ and the tabulation of the function $\operatorname{Gi}(z)=(1 / \pi) \int_{0}^{\infty} \sin \left(u z+\frac{1}{3} u^{3}\right) \mathrm{d} u$. Q. J. Mech. Appl. Maths 3, $107-112$.

Simakov, S. T. 1993 Initial and boundary value problems of internal gravity waves. J. Fluid Mech. 248, 55-65.

Simakov, S. T. 1994 Formation of singularities of limiting amplitude in a density stratified fluid disturbed by an extended monochromatic forcing. Wave Motion 19, 11-27.

Staquet, C. \& Riley, J. J. 1989 On the velocity field associated with potential vorticity. Dyn. Atmos. Oceans 14, 93-123.

Stevenson, T. N., Bearon, J. N. \& Thomas, N. H. 1974 An internal wave in a viscous heatconducting isothermal atmosphere. J. Fluid Mech. 65, 315-323.

Sutherland, B. R., Dalziel, S. B., Hughes, G. O. \& Linden, P. F. 1999 Visualization and measurement of internal waves by 'synthetic schlieren'. Part 1 . Vertically oscillating cylinder. J. Fluid Mech. 390, 93-126.

Sutherland, B. R., Flynn, M. R. \& Onu, K. 2003 Schlieren visualisation and measurement of axisymmetric disturbances. Nonlinear Process. Geophys. 10, 303-309.

Sutherland, B. R., Hughes, G. O., Dalziel, S. B. \& Linden, P. F. 2000 Internal waves revisited. Dyn. Atmos. Oceans 31, 209-232. 
Sutherland, B. R. \& Linden, P. F. 2002 Internal wave excitation by a vertically oscillating elliptical cylinder. Phys. Fluids 14, 721-731.

Thomas, N. H. \& Stevenson, T. N. 1972 A similarity solution for viscous internal waves. J. Fluid Mech. 54, 495-506.

VoIsIN, B. 1991 Internal wave generation in uniformly stratified fluids. Part 1. Green's function and point sources. J. Fluid Mech. 231, 439-480.

Watson, G. N. 1944 A Treatise on the Theory of Bessel Functions, 2nd edn. Cambridge University Press.

Wolfram, S. 1999 The Mathematica Book, 4th edn. Wolfram Media/Cambridge University Press. 
